\title{
The Associated Western Universities Summer Participant Program at the Lawrence Livermore National Laboratory Summer 1997
}

\section{Beverly Williams Student Internships Coordinator}

August 1997

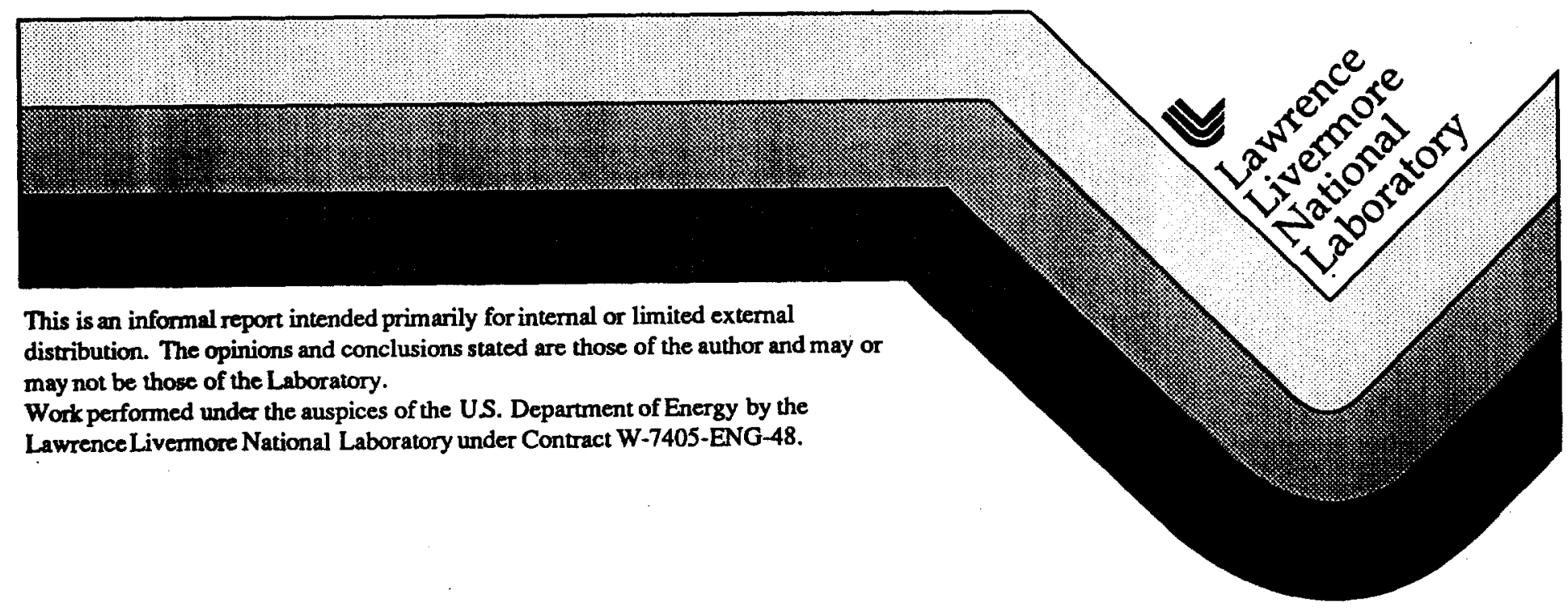




\section{DISCLAIMER}

This document was prepared as an account of work sponsored by an agency of the United States Government. Neither the United States Government nor the University of California nor any of their employees, makes any warranty, express or implied, or assumes any legal liability or responsibility for the accuracy, completeness, or usefulness of any information, apparatus, product, or process disclosed, or represents that its use would not infringe privately owned rights. Reference herein to any specific commercial products, process, or service by trade name, trademark, manufacturer, or otherwise, does not necessarily constitute or imply its endorsement, recommendation, or favoring by the United States Government or the University of California. The views and opinions of authors expressed herein do not necessarily state or reflect those of the United States Government or the University of California, and shall not be used for advertising or product endorsement purposes.

Work performed under the auspices of the U. S. Department of Energy by Lawrence Livermore National Laboratory under Contract W-7405-Eng-48. 


\section{The Associated Western Universities Summer 1997 Participant Program at}

Lawrence Livermore National Laboratory

\section{Authors}

Brian Beaubien

Emily A. Belli

Edgar D. Hardy

Eric D. Hempel

Matthew S. Hoehler

Jennifer Hollfelder

Carey Huscroft

Anabel Miranda

Shane Oram

Adam L. Rosenberg

Christen D. Rowe

Maureen Ryan

Matthew Torres

Timm Wulff

Affiliations: University of Minnesota Institute of Technology, Cornell University, Mississippi State University, Eastern Oregon University, Princeton University, Las Positas College, University of California, Davis, Turado University, Ithaca College, Cornell University, Smith College, University of South Carolina, California State University, Humboldt, California State University, Chico. 
Associated Western Universities, Inc. is an outgrowth of the Associated Rocky Mountain Universities, which was organized in the state of the Colorado in 1959. In 1967, the membership was expanded, the name was changed, and the charter was amended to create the current consortium which encompasses more than $30 \mathrm{DOE}$ research facilities and approximately 50 universities in the Western United States.

Lawrence Livermore National Laboratory has hosted AWU student participants every summer since 1968. In this time period (1968-1997) the AWU program provided approximately 563 fellowships to 365 students. The program has grown from three students in the summer of 1968 , to approximately 20 students each summer for the past twenty-nine years. Several students are awarded two consecutive fellowships. Many of these students continue their research projects during the academic year.

The students who have participated in the AWU-LLNL program are enthusiastically supportive of the research participation experience. Approximately three-fourths of the AWU alumni have gone on to postbaccalaureate education. More than a dozen of them have become permanent employees of Lawrence Livermore National Laboratory. The AWU-LLNL Honors Undergraduate Participation Program has helped the students by providing practical insight into the real world of research; developing communication skills; influencing educational goals; stimulating personal growth; giving focus for future life direction. It continues to be a unique learning experience for both students and supervisors alike. 


\section{THE ASSOCLATED WESTERN UNIVERSITIES SUMMER PARTICIPANT PROGRAM AT THE LAWRENCE LIVERMORE NATIONAL LABORATORY, SUMMER 1997}

\section{INTRODUCTION}

The Associated Western Universities, Inc. (AWU) ${ }^{1}$ supports a student summer program at Lawrence Livermore National Laboratory (LLNL). This program is structured so that honors undergraduate students may participate in the Laboratory's research program under direct supervision of senior Laboratory scientists. Typically, the new AWU participants are sophomores through seniors who are already functioning academically at the upper divisional level. Their superior abilities are indicated not only by their formal grade point averages, but also by their maturity, independence, verbal sophistication, intellectual curiosity, and their academic advancement beyond their class and credits. These students have longterm commitments to science. Their future plans include additional education leading to masters and doctoral degrees in such diverse fields as science, engineering, environmental programs, computations, medicine, and education.

At LLNL, the AWU participants work on a one-to-one basis with the senior scientists, and ideally, they return to LLNL for a second summer. Not infrequently, the results of the research of these second-and third-year students are published in scientific journals.

Included in this report is a list of the AWU participants for the summer of 1997 (Table 1a). All students are required to submit original reports of their summer activities in a format of their own choosing. These unaltered student reports constitute the major portion of this report.

1 Associated Western Universities, Inc.

Dr. Thomas G. Squires, Executive Director

4190 South Highland Drive, Suite 211

Salt Lake City, Utah 84124

801-273-8900

801-277-5632 (FAX) 


\section{HISTORICAL PERSPECTIVE OF AWU AT LLNL}

The Department of Energy, through its contractors, provides support for energy-related education and training programs at numerous research sites throughout the United States. These programs encompass training for persons employed at these sites for students from the universities who expect to continue in energy-related industry, and for college and university professors who are associated with the training and research work of the students.

The advanced student and faculty participation appointments are provided, in most instances, through the medium of regional organizations of universities. Examples of such organizations are the Associated Western Universities, the Oak Ridge Associated Universities, the Associated Universities, Inc., and the Argonne University Associates.

The Associated Western Universities serves the above purpose in most of the Western United States. Its overall objective is to promote and coordinate research and training on projects of a regional nature that require inter-university cooperation. In particular, it promotes training and research in energy-related science and engineering in the western part of the United States. Lawrence Livermore National Laboratory is one of the DOE sites open for participation by AWU faculty and students. University personnel are welcomed at DOE sites. DOE is interested in assisting the universities to expand and intensify their energy-related training and research programs through day-by-day work of university personnel (students and faculty) with the highly specialized DOE contractor staffs, usually on equipment not readily available at universities. Through this cooperative research program, the university participants not only advance their own needs, but eventually contribute substantially to the work of interest to the Department of Energy. The contributions may take many forms - training of future scientists; providing stimuli and suggestions for current work; furnishing ideas and suggestions for work to be done later on the university campuses. 


\section{OPERATION OF THE LLNL SUMMER PARTICIPANT PROGRAM}

Mr. Barry Goldman serves as the Internship Program Manager, and is assisted by Ms. Beverly Williams, Student Internship Coordinator and Ms. Helen Robinson, Education Assistant, at LLNL.

Mr. Barry Goldman

Manager, Internship Programs

Education Program/University Relations

Lawrence Livermore National Laboratory

P. O. Box 808, L-428

Livermore, CA 94551

(510) 422-5177; fax (510) 422-6079

email goldman $1 @ l l n l . g o v$

Ms. Beverly Williams

Student Programs

Education Program/University Relations

Lawrence Livermore National Laboratory

P.O. Box 808, L-428

Livermore, CA 94551

(510) 422-5020; fax (510) 422-6079

email williams92@llnl.gov

Ms. Helen L. Robinson

Internship Programs

Education Program/University Relations

Lawrence Livermore National Laboratory

P.O. Box 808 , L-428

Livermore, CA 94551

(510) 422-5377; fax (510) 422-6079

email robinson6@llnl.gov 


\begin{tabular}{|c|c|c|c|c|}
\hline Student & Institution & Major & $\begin{array}{l}\text { Academic } \\
\text { Rank } \\
1996-97\end{array}$ & $\begin{array}{l}\text { AWU } \\
\text { Participation } \\
\text { Years }\end{array}$ \\
\hline BEAUBIEN, Brian & $\begin{array}{l}\text { University of } \\
\text { Minnesota } \\
\text { Institute of } \\
\text { Technology }\end{array}$ & $\begin{array}{l}\text { Mechanical } \\
\text { Engineering/ } \\
\text { Biomedical } \\
\text { Engineering }\end{array}$ & Junior & 1997 \\
\hline BELLI, Emily A. & $\begin{array}{l}\text { Cornell } \\
\text { University }\end{array}$ & Engineering & Soph. & 1997 \\
\hline HARDY, Edgar D. & $\begin{array}{l}\text { Mississippi } \\
\text { State } \\
\text { University }\end{array}$ & $\begin{array}{l}\text { Civil } \\
\text { Engineering }\end{array}$ & Junior & 1997 \\
\hline HEMPEL, Eric D. & $\begin{array}{l}\text { Eastern } \\
\text { Oregon } \\
\text { University }\end{array}$ & Biology & Junior & 1997 \\
\hline HOEHLER, Matthew S. & $\begin{array}{l}\text { Princeton } \\
\text { University }\end{array}$ & Geology & Junior & 1997 \\
\hline HOLLFELDER, Jennifer & $\begin{array}{l}\text { Las Positas } \\
\text { College }\end{array}$ & $\begin{array}{l}\text { Computer } \\
\text { Science }\end{array}$ & Freshman & 1997 \\
\hline HUSCROFT, Carey & $\begin{array}{l}\text { University of } \\
\text { California, } \\
\text { Davis }\end{array}$ & Physics & Graduate & 1997 \\
\hline MIRANDA, Anabel & $\begin{array}{l}\text { Turado } \\
\text { University }\end{array}$ & Biology & Senior & 1997 \\
\hline ORAM, Shane & Ithaca College & Biology & Graduate & 1997 \\
\hline ROSENBERG, Adam & $\begin{array}{l}\text { Cornell } \\
\text { University }\end{array}$ & Engineering & Junior & 1997 \\
\hline ROWE, Christen D. & Smith College & Geology & Freshman & 1997 \\
\hline RYAN, Maureen & $\begin{array}{l}\text { University of } \\
\text { South Carolina }\end{array}$ & $\begin{array}{l}\text { Molecular } \\
\text { Biology }\end{array}$ & Soph. & 1997 \\
\hline TORRES, MATTHEW & $\begin{array}{l}\text { California State } \\
\text { University. } \\
\text { Humboldt }\end{array}$ & Biology & Senior & 1997 \\
\hline WULFF, Timm & $\begin{array}{l}\text { California State } \\
\text { University, } \\
\text { Chico }\end{array}$ & $\begin{array}{l}\text { Mecatronics } \\
\text { Engineering }\end{array}$ & Senior & 1997 \\
\hline
\end{tabular}




\title{
ASSOCIATED WESTERN UNIVERSITIES \\ Undergraduate Student Internship Program
}

\author{
Table of Contents
}

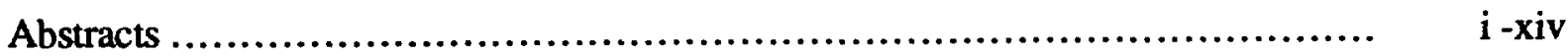

Brian Beaubien

Emily A. Belli

Edgar D. Hardy

Eric D. Hempel

Matthew S. Hoehler

Jennifer Hollfelder

Carey Huscroft

Anabel Miranda

Shane Oram

Adam L. Rosenberg The Yucca Mountain Project Small Block Tests..............

Christen D. Rowe Joint Inversion of Geophysical Data for Site Characterization and Restoration Monitoring

MTF Measurements of a Nova Gated X-ray Imager Using Film and a CCD Camera as the Recording Medium................

Rock Properties for Ground Motion Prediction at Yerba Buena Island

Overexpression and Purification of the Human DNA Repair Protein XPA

Construction of a Computer Model for Linear Analysis of Bixby Creek Bridge

Reservoir Induced Seismicity at Lake Del Valle, California ..

Quantum Monte Carlo Simulations of the Two-Band, 3d Hubbard Model.

Changes in Pore Water Chemistry as a Function of Microbial Growth in Yucca Mountain Tuff.

No Significant Evidence Found For a DNA Fingerprint from Densely Ionizing Radiation Based on F Ratios Calculated From Stable Chromosome Aberrations.

Maureen Ryan Radiocarbon Levels in Central and South San Francisco Bay

Matthew Torres

Transcript Mapping of Chromosome 19: Localization of THOP1, FTL, and LU genes.

100

Timm Wulff

Vacuum Control System for the Production Laser Cutting Work Station. 


\title{
Associated Western Universities \\ Summer 1997 Internship Program \\ at \\ Lawrence Livermore National Laboratory
}

\author{
Authors \\ Brian Beaubien \\ Emily A. Belli \\ Edgar D. Hardy \\ Eric D. Hempel \\ Matthew S. Hoehler \\ Jennifer Hollfelder \\ Carey Huscroft \\ Anabel Miranda \\ Shane Oram \\ Adam L. Rosenberg \\ Christen D. Rowe \\ Maureen Ryan \\ Matthew Torres \\ Timm Wulff
}

Affiliations: Brian Beaubien, University of Minnesota Institute of Technology; Emily A. Belli, Cornell University; Edgar D. Hardy, Mississippi State University; Eric D. Hempel, Eastern Oregon University; Matthew S. Hoehler, Princeton University; Jennifer Hollfelder, Las Positas College; Carey Huscroft, University of California, Davis; Anabel Miranda, Turado University; Shane Oram, Ithaca College; Adam Rosenberg, Cornell University; Christen D. Rowe, Smith College; Maureen Ryan, University of South Carolina; Matthew Torres, California State University, Humboldt; Timm Wulff, California State University, Chico. 


\title{
A 3-D FINITE ELEMENT MODEL OF THE HUMAN INDEX FINGER
}

\author{
Brian Beaubien \\ University of Minnesota - Institute of Technology \\ Computation
}

ABSTRACT

We are developing a hexahedral, finite element model of the human index finger for use in biomechanics research. Data obtained from high resolution CT scans are used to create 3-D surface definitions of bones in the hand. Hexahedral, volumetric meshes are created to match the soft tissue anatomy and bone surface definitions.

Materials and boundary conditions are then defined, and the mesh is processed for analysis in NIKE3D, a finite element code developed at LLNL.

Full flexion of the index finger has been accomplished, and we are currently working on the implementation of more accurate material models. Once complete, this model will be a valuable tool in prosthetics design, surgical planning, and biomechanics research and education. 


\title{
MTF Calculations for Gated X-ray Imagers Comparing Film and CCD Recording Media and Varying Camera System Properties
}

\author{
Emily Belli \\ Cornell University \\ Defense \& Nuclear Technologies
}

ABSTRACT

The modulation transfer function (MTF) represents the response of an imaging system to a sine-wave input as a function of spatial frequency. The usefulness of the MTF lies in that it is actually the Fourier transform of some function $f(x)$ such that the convolution of $f(x)$ with an idealized signal response $g(x)$ for a particular optical system yields the measured signal. This relation is represented as

$$
h(x)=f(x) * g(x)
$$

Using rectangular slits, as in this experiment, the idealized response $g(x)$ is a square wave. By measuring the square-wave response of the optical system $h(x)$, it is possible to calculate the contrast transfer function (CTF) of the system. The CTF $(r(n))$ can then be converted to the MTF $(R(n))$ using the relation

$R(n)=\pi / 4 *[r(n)+r(3 n) / 3-r(5 n) / 5+r(7 n) / 7-r(9 n) / n+\ldots](2)$

The Lawrence Livermore National Laboratory has designed and built a compact charge coupled device (CCD) camera system for use on the Six Inch Manipulator (SIM) at the Nova laser facility. This experiment shows that the $\mathrm{CCD}$ is suitable for replacement of T-max 3200 film as a recording medium in the X-ray Imagers. The MTF's for a Gated X-ray Imager with varying camera properties reveal that the signal to noise ratio using film as a recording medium is superior to that of the CCD cooled to $273.15 \mathrm{~K}$. However, when it is cooled to $258 \mathrm{~K}$, used without a fiber optic reducer, and used with a P-20 phosphor, the CCD's dynamic range is much greater than the peak resolution of the film with the $\mathrm{P}-11$ phosphor. Also, unlike film, the $\mathrm{CCD}$ yields a linear response and provides real-time access to the data. 


\title{
Rock Properties for Ground Motion Prediction at \\ Yerba Buena Island
}

\author{
Edgar Hardy \\ Mississippi State University \\ EPD, Geophysics \& Global Security
}

Earthquakes have been one of the leading natural hazards in California and particularly in the Bay Area where the disastrous Loma Prieta earthquake of 1989 occurred. This phenomenon has cost the Bay Area many human lives and millions of dollars in damage to buildings, bridges, and other structures. Scientists have researched the effects of previous earthquakes in order to predict the time, general area, and intensity of the next one that may occur.

One of the biggest projects in the Bay Area right now is the rebuilding of the east span of the Bay Bridge. Seismologists, structural and soil engineers, geophysicists, and other scientists are working with Caltrans (California Transportation) in order to build a safer, more flexible bridge that will be able to withstand the effects of a high-intensity earthquake. (Richter magnitude of $\sim 7.5$ on the Hayward fault)

Our project is a part of the geophysics and seismology research contributing to the overall Bay Bridge research. In our project we took rock samples supplied by Caltrans from Yerba Buena Island (the natural anchor for the Bay Bridge) and subjected them to earthquake conditions using a torsion oscillator. We measured and calculated such properties of the rock samples as strain, stress, and shear modulous (stiffness) under these conditions.

Using measurements of the strain dependence of rock properties we can understand the behavior of the rock during an earthquake and hopefully predict how it will behave during the next earthquake. 


\title{
Overexpression and Purification of the Human DNA Repair Protein XPA
}

\author{
Eric D. Hempel \\ Eastern Oregon University \\ Biology and Biotechnology Research Program
}

\section{ABSTRACT}

The ability of human cells to recognize, excise, and repair a variety of damaged sites in DNA, resides in a relatively small protein. Xeroderma pigmentosum group $\mathrm{A}$ (XPA) is a $38-42 \mathrm{kDa}$ zinc finger protein that contains a DNA binding domain. A deficiency in XPA results in a rare genetic disease, xeroderma pigmentosum, which is characterized by hypersensitivity to sunlight. Symptoms include massive skin cancer tumors and neurological disorders. This project focused on producing pure human XPA protein, so that it can be studied in the future. I developed a protocol for overexpression of human XPA in Escherichia coli and its purification to near homogeneity using affinity and gel filtration column chromatography methods. The final yield was about $6 \mathrm{mg}$ of purified XPA obtained from a 1-liter culture of cells. Final purity was qualitatively approximated by a single band on a denaturing polyacrylamide gel. 


\title{
Reservoir Induced Seismicity at Lake Del Valle, California
}

\author{
Jenny Hollfelder \\ Las Positas College \\ EPD, Geophysics and Global Security
}

Reservoir Induced Seismicity (RIS) is the triggering of earthquakes by the disturbance of an area following the filling or fluctuations in water volume of a reservoir. RIS can be the result of a rapid increase of elastic stress or the gradual diffusion of water to hypocentral depths (Simpson, Leith, and Scholz 1988). Because the volume of water in a reservoir affects both of these situations by increasing the pressure on the ground, I collected reservoir volume and earthquake event data for Lake Del Valle between the years of 1979 and 1996. My goal was to find a correlation between the filling of the reservoir and the number of earthquakes occurring within the same time period. My method of comparison was a combination graph using the water volume and number of events as variables and time as a constant. 


\title{
Quantum Monte Carlo Simulations of the Two-Band, 3d Hubbard Model
}

\author{
Carey Huscroft \\ University of California, Davis \\ Physics \& Space Technology
}

\section{ABSTRACT}

The two-band, 3d Hubbard Model with inter-site hybridization between the two bands is a model which may be used to study phenomena in materials in which the effects of strongly correlated electrons both within and between bands are important. An example is the pressure-induced volume-collapse phase transition in certain lanthanide and actinide materials. Although the phase diagram of this model has long been of interest, a thorough study of this model using Quantum Monte Carlo techniques is not feasible using current vector computer architectures. We use approximation-free Auxiliary-Field Quantum Monte Carlo (AFQMC) techniques on massively-parallel supercomputers to perform the first extensive AFQMC study of the phase diagram of this model. We map out the crossover regime wherein Kondo singlets are formed as the hybridization is increased. We also investigate the magnetic properties of this model. We discuss where LDA-derived parameters place $\mathrm{Ce}$ and other rare earths on the phase diagram. 


\title{
Changes in Pore Water Chemistry as a Function of Microbial Growth in Yucca Mountain Tuff
}

\author{
Anabel Miranda \\ Turado University \\ EPD, Geophysics \& Global Security
}

\begin{abstract}
\end{abstract}
The US Department of Energy has been charged with the design and construction of a nuclear waste repository. This repository must safely contain these wastes over a period of at least 10,000 years. The location of the repository has been proposed to be at Yucca Mountain (YM), Nevada, below the ground surface in welded volcanic ash tuff. The purpose of this study is to determine effects of microbial growth in YM tuff pore water chemistry and metal stability to determine the chemical impact of microbial activity in a tuff and tuff + metal environment as a function of macronutrient supply. To distinguish between abiotic vs. biotic chemistry, it will be necessary to sterilize the YM tuff used in these experiments. The goal of the work described here is to sterilize tuff obtained from Exploratory Studies Facility (ESF Yucca Mountain, Nevada) without substantially altering tuff chemistry. The tuff is treated using the following: antibiotics, sodium azide, heat, sonication, $\mathrm{HgBr} 2$, dry heat, and the combination of sodium azide and antibiotics. The treatments that show no growth and no chemical changes after sterilization, will be candidate sterilization methods for sterile controls in this experiment. 


\title{
Chromosome Damage Caused by Radiation
}

\author{
S. W. Oram \\ Ithaca College \\ Biology \& Biotechnology Research Program
}

\begin{abstract}
The purpose of this study is to measure the ratio of translations to pericentric inversions for low-LET radiation and high-LET radiation using fluorescence in situ hybridization (FISH). A composite chromosome $1 \mathrm{p}$ telomeric region specific probe, a chromosome 1 heterochromatin probe and a pan-centromere probe were developed to identify pericentric inversions. A chromosome 1 paint probe was employed to measure translocations on chromosome 1 as described by Lucas et al. (1989, 1992). Translocation frequencies and pericentric inversion frequencies were calculated as described by Lucas et al. (1989, 1996).
\end{abstract}

Translocations are interchromosomal aberrations (breaks in different chromosomes), while pericentric inversions are intrachromosomal aberrations (breaks in the same chromosome). High-LET radiation produces energy depositions that are much closer together (dense) than those produced by low-LET radiation (sparse). Hence, it may be more likely for high-LET radiation to cause multiple breaks in the same chromosome than it is for low-LET radiation. If this is true, then it is expected that the ratio of translocations to pericentric inversions(F-Ratio) for high-LET radiation will be less than the FRatio for low-LET radiation, therefore facilitating a "fingerprint" for high-LET radiation (Brenner, 1996).

Contrary to prediction, there does not appear to be a "fingerprint" for high-LET radiation. The F-Ratios found for high- and low-LET radiation were similar, with the high-LET radiation being slightly lower. 


\title{
Construction of a Computer Model for Linear Analysis of Bixby Creek Bridge
}

\author{
Matthew S. Hoehler \\ Princeton University \\ EPD,Geophysics \& Global Security
}

\section{ABSTRACT}

In the past few years the state of California has engaged in an all-out effort to safeguard its critical infrastructure from seismic hazards. This report is one component of a study to be performed by Lawrence Livermore National Laboratory (LLNL) for the California Department of Transportation ( Caltrans). The study will investigate the effects of earthquakes on deck-type arch bridges with the intent to characterize the behavior of these structures during seismic events. Structure behavior characterization allows for the development of adequate seismic retrofit measures.

The scope of the study includes performing linear and nonlinear earthquake analysis of Bixby Creek Bridge incorporating the empirical Green's functions method to generate realistic, site-specific ground motion. Bixby Creek Bridge was chosen for the study because it is a fairly large, concrete deck-type arch bridge and it is a critical link along US Highway 1. Bixby Creek Bridge is representative of a number of other concrete arch bridges built during the 1930's.

This report documents the construction and modeling details of a computer model generated for linear analysis using the GEMINI finite element analysis program. The structure's critical natural frequencies and mode shapes are documented herein. These critical frequencies will be used to determine a range of frequencies pertinent to the structure to aid the collection of the microearthquake data used by the empirical Green's function method. 


\title{
The Small Block Experiment for the Yucca Mountain Project
}

\author{
Adam Rosenberg \\ Cornell University \\ EPD, Geophysics \& Global Security
}

\begin{abstract}
This report documents progress on laboratory testing of the third small block samples of Topopah Spring tuff. The purpose of these tests is to investigate the coupled thermal-mechanical-hydrologicalchemical response of the rock to conditions similar to the near-field environment (NFE) of a potential nuclear waste repository. This report contains preliminary results for flow measurements on a $0.5 \mathrm{~m}$ scale block of Topopah Spring tuff containing a horizontal, throughgoing artificial fracture, and several smaller natural fractures and vugs. Results of these tests will provide insight into the processes controlling fluid flow in fractured rocks at temperature and stress conditions appropriate to the Near-Field Environment, and will contribute to the database used in simulations of the potential repository.
\end{abstract}

We have performed an experiment on a $0.5 \mathrm{~m}$ scale block of tuff containing an artificial fracture. Fluid flow in the fracture was generated by a point source in the plane of the fracture at its center. This created a radial flow field to probe the effect of anisotropy of the rock fabric on the flow in the fracture. The perimeter of the fracture was divided into 38 intervals and fluid flow was monitored for each interval. Different water pressures and stresses on the rock fracture are currently being tested. Results so far show a tendency towards almost all flow entering about three or four specific jars around the block, but after those entries are closed off, a regular pattern can be seen in where the flows will appear next. Flow rate out of the rock increases with water pressure and slightly decreases with axial stress. Water in and water out plots seem to be fairly linear and parallel through time so far.

Displacement in the rock, especially across the fracture is also being monitored, and while there is certainly a decrease in fracture width with increasing axial stress, more data is needed to achieve a good approximation for the relationship. 


\title{
Joint Inversion of Geophysical Data for Site Characterization and Restoration Monitoring
}

\author{
Christie D. Rowe \\ Smith College \\ EPD, Geophysics \& Global Security
}

\section{ABSTRACT}

Current characterization techniques for polluted ground sites, to be effective, require drilling wells, an expensive process which may increase the spreading of contaminants. Various non-intrusive geophysical data collection methods are currently in use, but none is as reliable or informative as data collected from wells and core samples. The Joint Inversion project aims to combine two methods of non-intrusive data collection, seismic and electrical methods. We will combine the two methods via a computer code, which will predict properties of the earth such as porosity and saturation based on ultrasonic velocity and resistivity of the site. Our computer code will replace the traditional bore hole data collection methods. 


\title{
Radiocarbon Levels in San Francisco Bay
}

\author{
Maureen Ryan \\ University of South Carolina \\ Center for Accelerator Mass Spectrometry
}

\section{ABSTRACT}

The radioactive isotope ${ }^{14} \mathrm{C}$ occurs in nature, but over time anthropogenic events such as the industrial revolution and atomic testing have altered these natural levels. Measurements of ${ }^{14} \mathrm{C}$ levels in organisms can be used for a variety of applications in an array of the natural sciences, including the monitoring of the bioavailability of petroleum-based pollutants. In order for such a study to be conducted, the naturally occurring levels of radiocarbon must be known.

By utilizing Accelerator Mass Spectrometry technology, a survey of the ${ }^{14} \mathrm{C}$ levels in marine organisms of San Francisco Bay was conducted at 5 sites around the Bay. Organic and calcareous samples were collected, pretreated, converted to graphite targets, and the ${ }^{14} \mathrm{C}$ levels determined through AMS.

An increase in ${ }^{14} \mathrm{C}$ levels was observed for South Bay sites in comparison to Central Bay sites. These increases can most likely be attributed to decreased freshwater flow, increased exposure to atmospheric $\mathrm{CO}_{2}$, and increased municipal waste input into the South Bay waters. The data produced correlates well with a previous study on $\Delta^{14} \mathrm{C}$ levels in the Bay. 


\title{
Transcript Mapping of Chromosome 19 with Expressed Sequence Tags (ESTs): Localization of the Lutheran blood group glycoprotein (LU).
}

\author{
Matthew Torres \\ California State University, Humboldt \\ Biology and Biotechnology Research Program
}

\begin{abstract}
Approximately $95 \%$ of chromosome 19 (excluding the centromere) is covered by cosmid contig maps assembled by automated restriction enzyme analysis. Roughly 400 different genes have been localized to these specific cosmids, 300 of which have been incorporated into the ordered map. Still, many known genes have yet to be localized to specific cosmid contigs; and moreover, many unknown genes have yet to be discovered. Expressed Sequence Tags (ESTs), partial cDNAs generated from mRNA libraries and cloned into bacterial hosts, have become a useful tool for the discovery and localization of these genes. The large database of EST sequences in dbEST can be easily compared to any specific sequence for the selection of an EST clone that matches a unique probe. Specifically, we used a short sequence of the Lutheran blood group glycoprotein (LU) gene (known to be present on chromosome 19) to probe dbEST. Human Lutheran glycoproteins are surface antigens found in erythrocyte membranes and possibly responsible for signal-transduction. One of the matching ESTs was used to screen a cosmid library of Chromosome 19. Hybridization positives were verified by $P C R$ and were localized to specific cosmids on the restriction-fragment contig map constructed at LLNL. Results confirm that the LU gene maps to chromosome $19 \mathrm{q} 13.2$.
\end{abstract}




\title{
Vacuum Control System for the Laser Cutting Work Station
}

\author{
Timm Wulff \\ California State University, Chico \\ Electronics Engineering, \\ Computer \& Communication, \\ Engineering Division
}

ABSTRACT

A first of its kind Laser Cutting Work Station (LCWS) is currently in production at LLNL that utilizes a new laser technology. Of the variety sub-systems in the LCWS, the vacuum system provides an environment where cutting is performed. The quality of the cut is directly related to the degree of vacuum. Achieving control of the system was done using Lab View (a visual programming language). Lab View also provides the user interface that LCWS operators will use in application. The code that operates the system was designed to allow the operator to give one single command to perform a vast number of steps sequentially to achieve pumping down to vacuum and bleeding up to atmospheric pressure. There are also options for controlling the system manually. All user commands are guarded with safety interlocks to prevent mishaps from occurring. The software is also designed flexible and can adapt to changes in PLC addressing and easily accommodates new devices that may be added in the future. 


\title{
A 3-D FINITE ELEMENT MODEL OF HUMAN INDEX FINGER FLEXION
}

\author{
Brian Beaubien \\ Supervisor: Karin Hollerbach \\ Building 319, Room 154, L-416 \\ Lawrence Livermore National Laboratory, University of California \\ Livermore, California 94550
}

Current laboratory testing methods cannot provide adequate information on finger joint biomechanics. We are developing a hexahedral, finite element model that will provide this information without interfering with the natural joint kinematics. Using this model, we aim to quantify the stresses and strains experienced by the bones, tendons, and ligaments during natural flexion of the finger. Our results will be important in describing the joint mechanics and in designing and testing prosthetic devices such as the MP joint implant.

Our model begins with structural bone data acquired on site at Non-Destructive Evaluation (NDE) with an industrial CT scanner. Scans with 160 micron spatial resolution were taken of the cadaver hand of a 53 year old Caucasian female. Bone was segmented from the scans semi-automatically using VISU (NDE), which allows interactive manipulation of scanner artifacts and bone deterioration. Polygonal surface definitions were then compiled from the scans and used to generate hexahedral, volumetric meshes in the TrueGrid pre-processor (XYZ Scientific).

The model includes all index finger bones, true and accessory collateral ligaments, annular pulleys, and flexor tendons. Bones are represented as rigid bodies, but will soon be homogeneously orthotropic elastic, and cortical and cancellous bone will eventually be differentiated. Annular pulleys are modeled as strictly elastic, and each has an elastic modulus (E) of $400 \mathrm{MPa}$, and poisson's ratio (v) of .36. A thermal-elastic material was used for the ligaments to allow thermal pre-tensioning, followed by strictly elastic 
behavior. Material properties for the metacarpo-phalangeal (MP) and proximal and distal interphalangeal (PIP and DIP) collateral ligaments are as follows:

\begin{tabular}{|c|c|c|c|}
\cline { 2 - 4 } \multicolumn{1}{c|}{} & $E(\mathrm{MPa})$ & $\mathrm{v}$ & Coeff. of thermal exp. \\
\hline MP Joint & 400 & 0.46 & 0.001 \\
\hline DIP Joint & 100 & 0.46 & 0.001 \\
\hline PIP Joint & 200 & 0.46 & 0.001 \\
\hline
\end{tabular}

The flexor digitorum profundus (FDP) and flexor digitorum superficialis (FDS) both use a transversely isotropic, hyperelastic material capable of modeling a fiber-reinforced rubber. Their material properties closely match those of human ligaments ${ }^{1}$ and their material coefficients are as follows:

\begin{tabular}{|c|c|c|c|c|c|c|}
\hline$C_{1}$ & $C_{2}$ & $C_{3}$ & $C_{4}$ & $C_{5}$ & $k$ & lambda \\
\hline 11.0 & 0.0 & 2.2 & 78.8 & 887.6 & $500 \mathrm{MPa}$ & 1.02 \\
\hline
\end{tabular}

where $C_{1}$ and $C_{2}$ are Mooney-Rivlin rubber coefficients, $C_{3}$ scales the exponential stresses, $\mathrm{C}_{4}$ is the rate of uncrimping of collagen fibers, $\mathrm{C}_{5}$ is the modulus of straightened collagen, $\mathrm{k}$ is the bulk modulus, and lambda is the fiber stretch.

Quasi-static analysis is performed using the NIKE3D (LLNL) code running on Silicon Graphics Indigo ${ }^{2}$ and Indy workstations. The entire index finger mesh contains 25,692 nodes, and 18,971 eight noded elements. The metacarpal was fully constrained in translation and rotation, and the proximal tip of the FDS and FDP were displaced $2.75 \mathrm{~cm}$ and $3.25 \mathrm{~cm}$ respectively. All of the model's contact interfaces were frictionless.

We have achieved full flexion of the index finger model, and are making improvements to provide more quantitatively relevant results. The extensor digitorum tendon will be added to the mesh, and material properties will be modified to match published values. Stresses, Strains, and displacements will then be determined and compared with published empirical data. The finished model will be a valuable tool for kinematics research, and for the testing of MP joint implants.

1Weiss, J.A., "A Model for the Material Behavior of Ligaments and Tendons," ASME Advances in Bioengineering Vol.31, 97-98 (1995). 


\title{
MTF Measurements of a Nova Gated X-ray Imager Using Film and a CCD Camera As the Recording Medium
}

\author{
E.A. Belli, L.M. Logory, D.R. Farley, \& P.L. Miller \\ Supervisor: David R. Farley \\ Building 111; Room 273; L-022 \\ Lawrence Livermore National Laboratory \\ Livermore, California 94551
}

\section{Introduction}

In these experiments we have measured the modulation transfer function (MTF) of an x-ray imaging system which is routinely used on the Nova laser. The MTF represents the response of an imaging system to a constant amplitude, sine-wave input as a function of spatial frequency. The MTF indicates the resolution and contrast of the image, the amount of perceivable detail within the image, and the sharpness of the image over the expected spatial frequency domain of the instrument. The usefulness of the MTF lies in that it is the Fourier transform of some function $f(x)$ such that the convolution of $\mathrm{f}(\mathrm{x})$ with an idealized signal response $\mathrm{g}(\mathrm{x})$ for a particular optical system yields the measured signal. This relation is represented as

$$
h(x)=f(x)^{*} g(x)
$$

Here, however, we choose to measure the contrast transfer function (CTF) because it is difficult to generate a sine wave input $x$-ray signal which would be required to measure the MTF directly. The CTF is similar to the MTF with the exception that it measures the 
The gated x-ray imager (GXI) is used as a diagnostic at the Nova laser facility at the Lawrence Livermore National Laboratory. The spatial frequency response of this imager is of interest to researchers because it determines the amount of obtainable detail within experimental data. Measurements of the imager MTF (Modulation Transfer Function) were made on a static $\mathrm{x}$-ray source and are reported on here. These measurements do not include the effects of magnification snouts and pinholes since the primary goal here was to compare different image recording media. Two recording media were chosen for this study; Kodak T-max 3200 film, and a scientific grade CCD electronic camera. The measurements show that T-max film has an MTF approximately 30 percent larger than the $\mathrm{CCD}$ camera for all spatial frequencies. However, measurements for these two media indicate that the signal-to-noise ratio $(\mathrm{S} / \mathrm{N})$ for the film is nearly three times smaller than that obtained by the CCD. Future measurements and improvements to the CCD camera system will also be discussed.

\section{Introduction}

In these experiments we have measured the modulation transfer function (MTF) of an x-ray imaging system which is routinely used on the Nova laser. The MTF represents the response of an imaging system to a constant amplitude, sine-wave input as a function of spatial frequency. The MTF indicates the resolution and contrast of the image, the amount of perceivable detail within the image, and the sharpness of the image over the expected spatial frequency domain of the instrument. The usefulness of the MTF lies in that it is the Fourier transform of some function $f(x)$ such that the convolution of $f(x)$ with an idealized signal response $g(x)$ for a particular optical system yields the measured signal. This relation is represented as

$$
h(x)=f(x) * g(x)
$$

Here, however, we choose to measure the contrast transfer function (CTF) because it is difficult to generate a sine wave input $\mathrm{x}$-ray signal which would be required to measure the

MTF directly. The CTF is similar to the MTF with the exception that it measures the 
response of an imaging system to a square-wave input. The CTF data $(\mathrm{r}(\mathrm{n}))$ can be mathematically converted to an equivalent MTF response (R(n)) using the relation

$$
R(n)=\pi / 4 *[r(n)+r(3 n) / 3-r(5 n) / 5+r(7 n) / 7-r(9 n) / 9+\ldots]
$$

A derivation of this formula from Fourier transform analysis can be found in Appendix A.

Figure 1(A) shows a constant amplitude, square wave input such as that which would be produced if a uniform, constant amplitude source were directed toward a series of slits with increasing spatial frequency and decreasing slit width. Figure 1(B) shows the resulting output wave that would be measured by an imaging system. It is notable that as the spatial frequency increases, the observable contrast within the image decreases. In other words, the maximum and minimum signals converge.

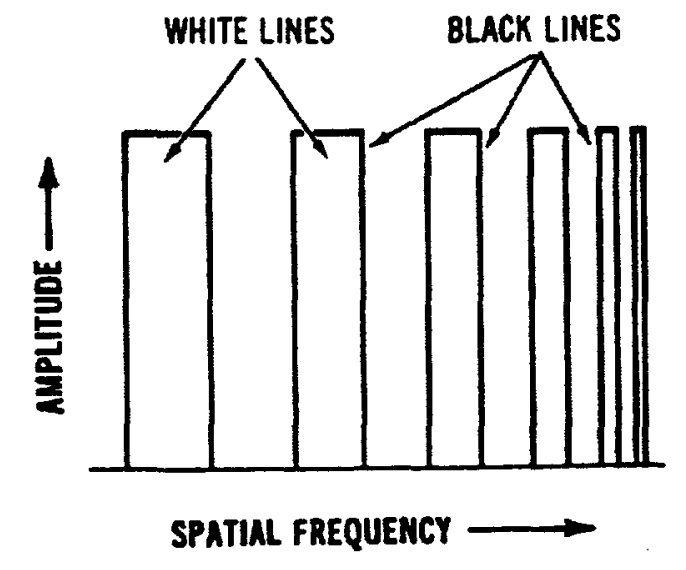

(A) Input wave.

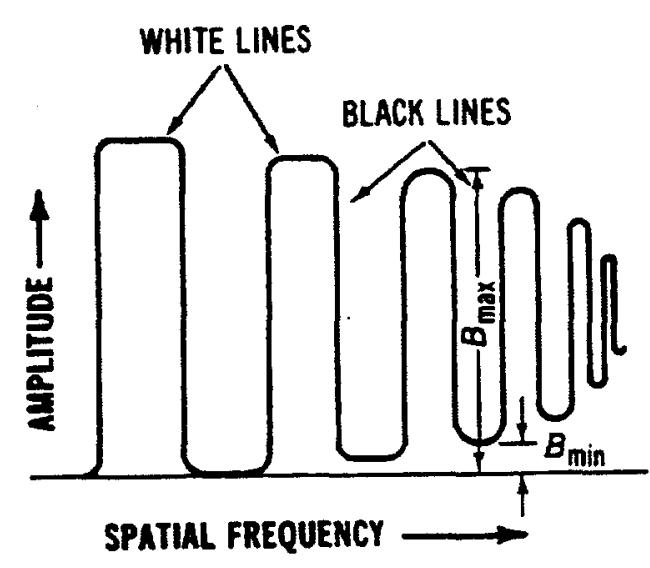

(B) Output wave.

Figure 1. a) A constant amplitude, square wave input and b) the measured signal that would be expected from a given imaging device.

\section{CTF Mask Design}

A mask containing rectangular slits of various spatial frequencies has been designed and fabricated to measure the CTF. The mask consists of a $100 \mu \mathrm{m}$ thick silicon 
wafer with a $1 \mu \mathrm{m}$ gold coating on both sides to prevent $\mathrm{x}$-rays from traveling through the mask in areas where slits are not present. The mask has 19 sets of 8 slits with the width between the slits varying from set to set. The maximum slit width is 22.5 line pairs per millimeter $(\mathrm{LP} / \mathrm{mm})$, and the minimum slit width is $0.5 \mathrm{LP} / \mathrm{mm}$. The design and a photograph of the mask are shown below in Figure 2.

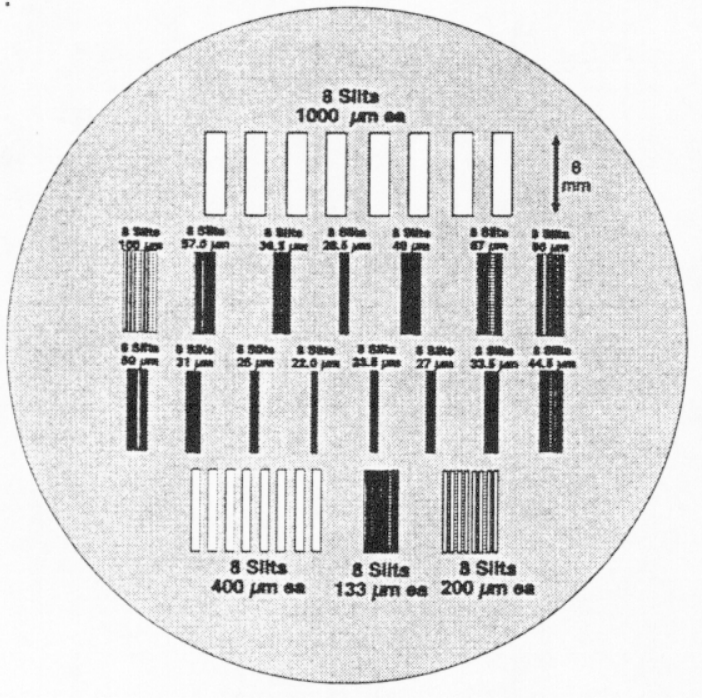

(a)

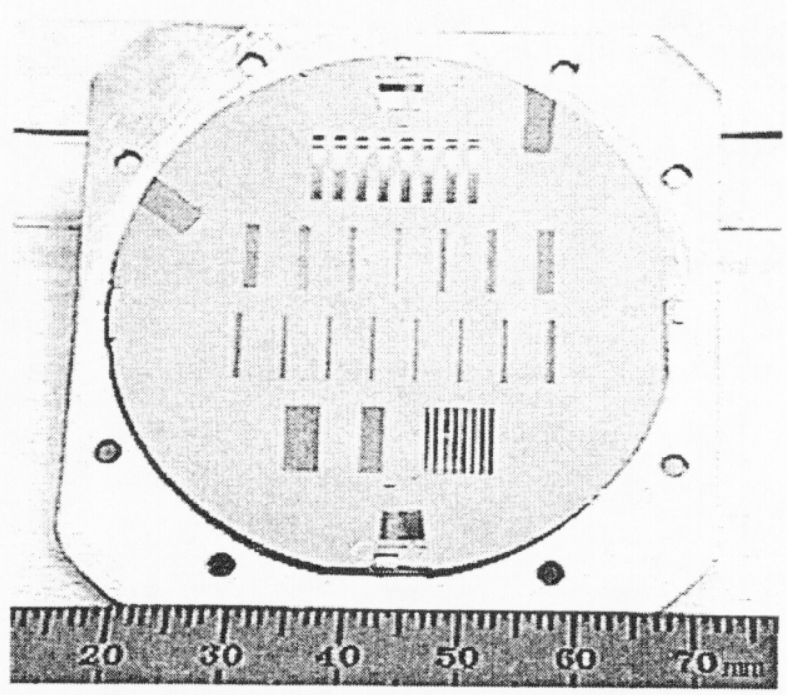

(b)

Figure 2. (a) The mask design and (b) a photograph of the fabricated part.

The mask was examined for edge defects with a scanning electron microscope and is shown in Figure 3. The slit edges are high quality, i.e. very rectangular, which is important for creating the square wave input necessary for making the CTF measurements. 


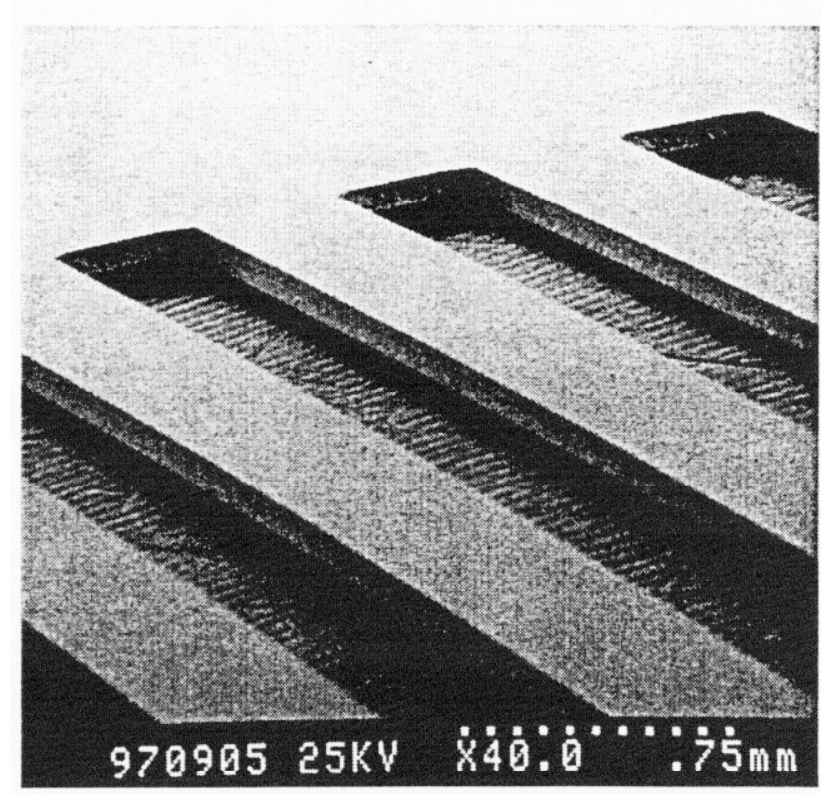

Figure 3. A high-magnification image of the CTF mask showing the $1.25 \mathrm{LP} / \mathrm{mm}$ slits.

\section{Experimental Setup}

The experimental setup consists of an x-ray source, a $1.3 \mathrm{~m}$ extension tube, and the gated $x$-ray imager. The entire setup, with the exception of the recording medium and support electronics, is held under a high vacuum at a pressure of about $10^{-7}$ torr. The $\mathrm{x}$ ray source uses a cathode on which a current is applied to boil off electrons which are accelerated to a titanium anode to produce $4.7 \mathrm{KeV}$ photons. An extension tube, which connects the $\mathrm{x}$-ray source to the imager, was added to reduce the divergence angle of the $\mathrm{x}$ ray photons as imaged on the microchannel plate detector in the imager. This is necessary in order to produce the most accurate representation of the slit patterns on the image plane. A schematic diagram of the experimental setup is shown in Figure 4. 


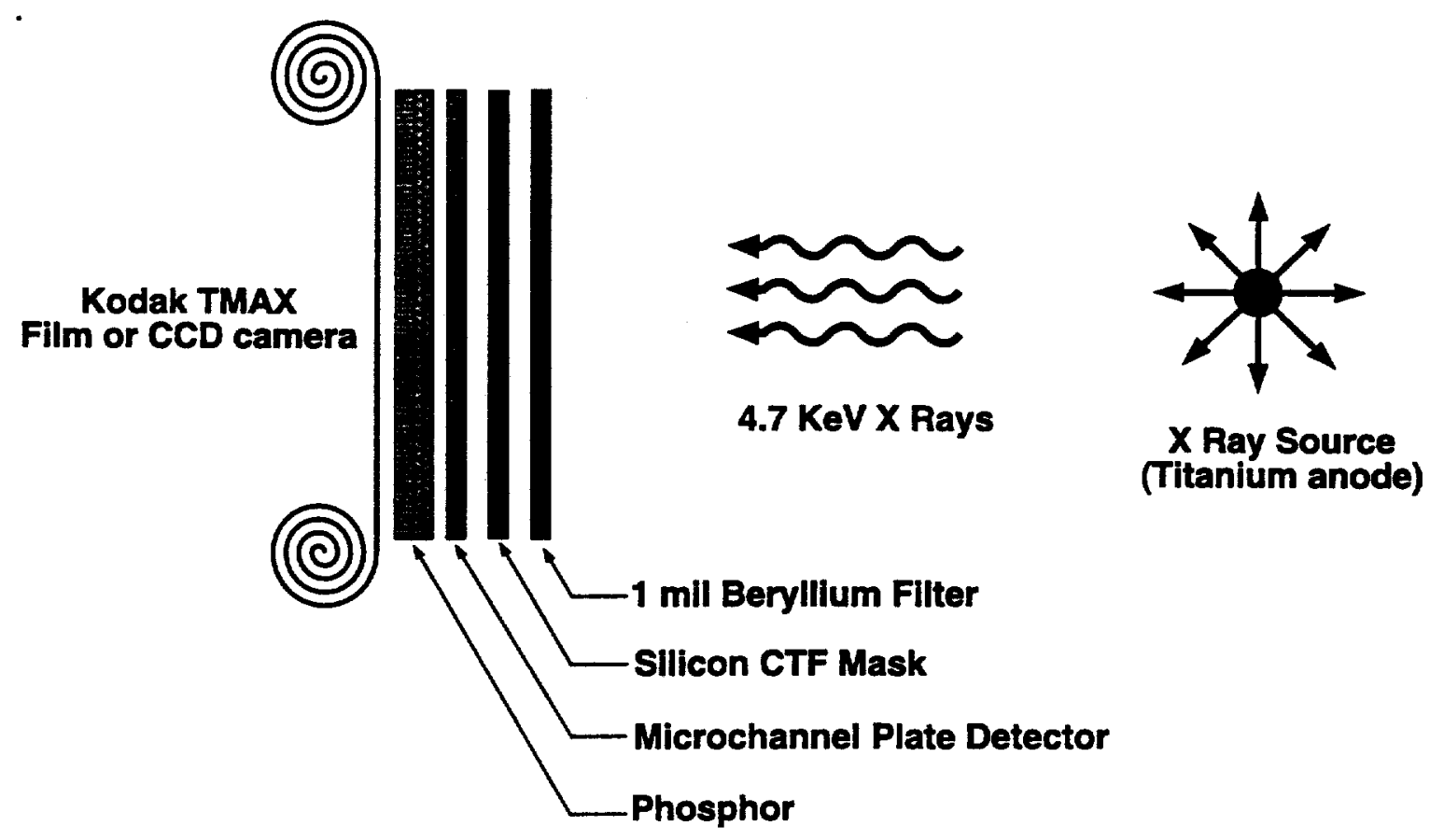

Figure 4. A schematic diagram of the experiment showing the $\mathrm{x}$-ray source and the internal components of the gated $x$-ray imager (GXI).

The current design of the gated $\mathrm{x}$-ray imager (GXI) consists of a series of imaging devices followed by support electronics, as shown in Figure 5. The x-rays enter the front of the GXI through a $25 \mu \mathrm{m} \mathrm{Be}$ filter. This filter not only protects the camera from debris, but it also blocks extraneous UV light. X-ray photons travel through the slits of the CTF mask to the microchannel plate (MCP) where they are converted into electrons. The MCP is a gold-coated plate with four parallel silicon strips across it. In this experiment, voltage from a DC source is applied to the strips. The electrons are knocked off of the MCP as x-rays interact with the strips and are accelerated to a P-20 phosphor by a $3 \mathrm{KV}$ potential difference. The phosphor, mounted on a fiber optic face plate, converts the electrons into optical light. Finally the image is captured on a recording medium, such as T-MAX 3200 optical film or a CCD camera. 


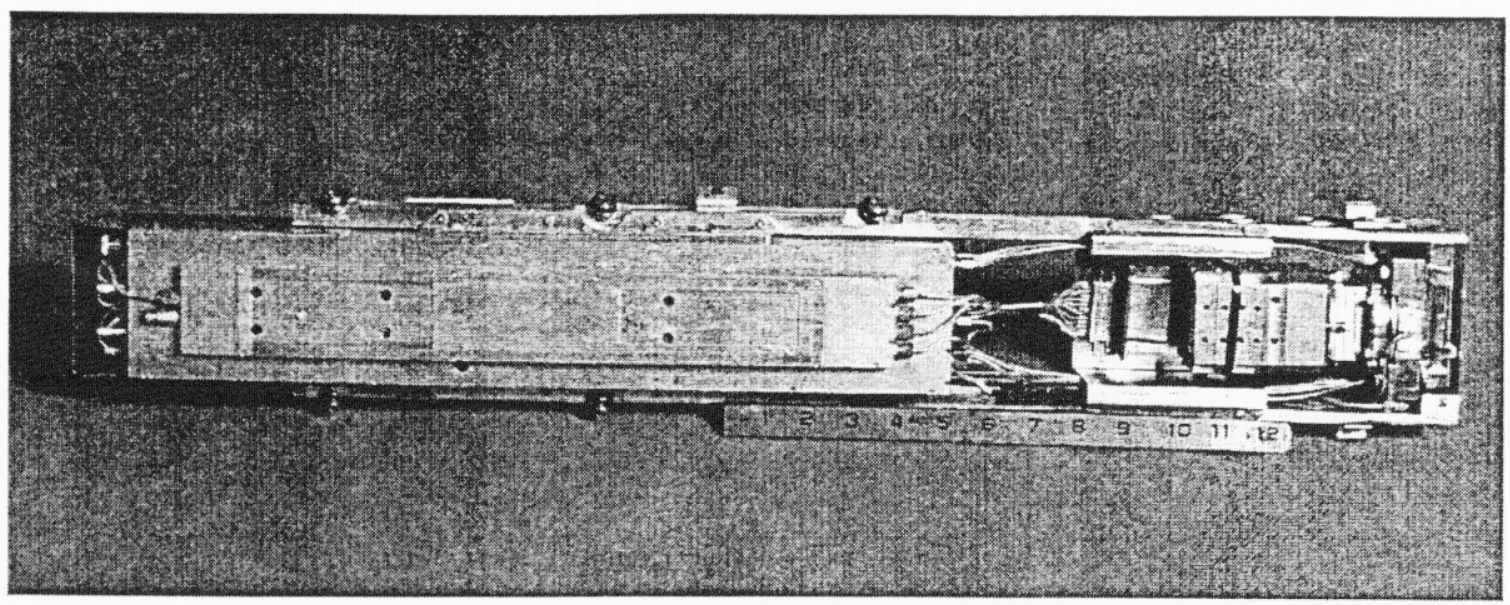

Figure 5. A photograph of the gated $x$-ray imager configured with the CCD camera.

The CCD array used here consists of $24 \mu \mathrm{m}$ square pixels with an image format of $1024 \times 1024$ pixels. The physical dimensions of the CCD array are smaller than the output phosphor, thus requiring a fiber optic reducer for imaging. The camera controller digitizes the image to 16 bits and transmits the data to a personal computer via a serial data link at a rate of $150 \mathrm{kHz}$. The CCD array is currently being operated at $-45^{\circ} \mathrm{C}$ in order to reduce the production of thermal electrons on the surface of the CCD camera. A close-up photograph of the current CCD camera configuration is shown in Figure 6.

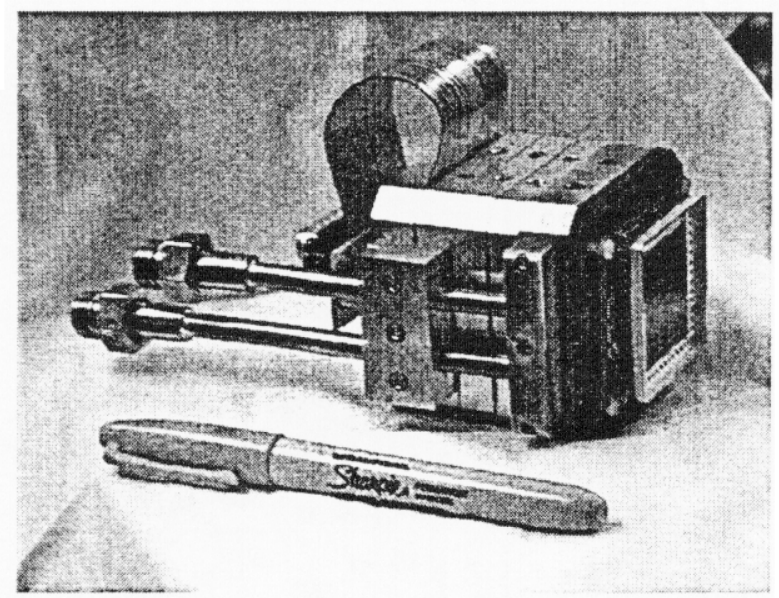

Figure 6. A photograph of the CCD camera. shown here with its support electronics and coolant lines. 


\section{Experimental Procedure}

The experiments were conducted with film and a CCD camera as the recording medium. For the film, three different mask orientations were tested to examine the uniformity of the microchannel plate. With the CCD camera, measurements were made both with and without the fiber optic reducer which couples the CCD camera to the phosphor. The procedure is shown below in Table 1 .

Table 1. CTF mask orientation and recording media used in the experiments.

\begin{tabular}{|l|l|l|}
\hline Medium & Film & CCD \\
\hline Standoff Distance & $6 \mathrm{ft}$. & $6 \mathrm{ft}$. \\
\hline Coupling & n/a & Reducer \& No Reducer \\
\hline Phosphor & P-20 & P-20 \\
\hline Mask Orientation & $0,90,180$ degrees & 0 degrees \\
\hline
\end{tabular}

\section{Analysis Techniques}

The images were analyzed using the Interactive Data Language program and a set of routines known as AIM, written by Juan Moreno. The film data was scanned in with a $5 \mu \mathrm{m}$ aperture. A P-20 wedge was used to convert the data from film density to film exposure (a linear scale). The CCD data was read in as photon counts. The data was analyzed by taking a lineout of the image through each set of slit patterns. This yielded a plot of exposure (or counts) vs. position. from which the maximum and minimum levels were identified for each spatial frequency. The five middle maxima and their successive minima (out of eight) were averaged to get one maximum and one minimum for each spatial frequency. The CTF was then calculated using the formula, (Bmax - Bmin)/(Bmax 
$+B \min$ ), where $B \max$ is the maximum light intensity corresponding to a slit and $B \min$ is the minimum intensity.

The MTF was calculated using equation 2. The data used in the equation were obtained from the curve fit through the CTF data points at each spatial frequency. Double gaussian curves were fit to both the CTF and MTF data.

The signal to noise ratio was calculated for each recording medium. This was accomplished by dividing the mean image values by the standard deviation within a uniformly illuminated region within the image. The same image areas were used for comparison of the film and CCD images.

\section{Results \& Discussion}

The measured data has been compiled into a series of plots of the CTF and MTF vs. spatial frequency for all mask orientations and recording medium combinations. A CTF or MTF value of 1.0 indicates perfect contrast. Figure 7 shows the CTF and MTF curves for data taken with film as the recording medium and with the mask oriented at 0 degrees, 90 degrees, and 180 degrees to examine the uniformity of the microchannel plate. The similarity of the 0 degrees and 180 degrees plots suggests that the MCP is uniform across its strips. However, the discrepancy from these curves seen in the 90 degrees plot shows that the MCP has some directional dependence with regard to its MTF. This is possibly due to an anisotropic electric field within the MCP strips. Figure 8 compares data taken with both film and the CCD with and without the fiber optic reducer. The data from the CCD camera shows substantially less contrast than that obtained with film. 


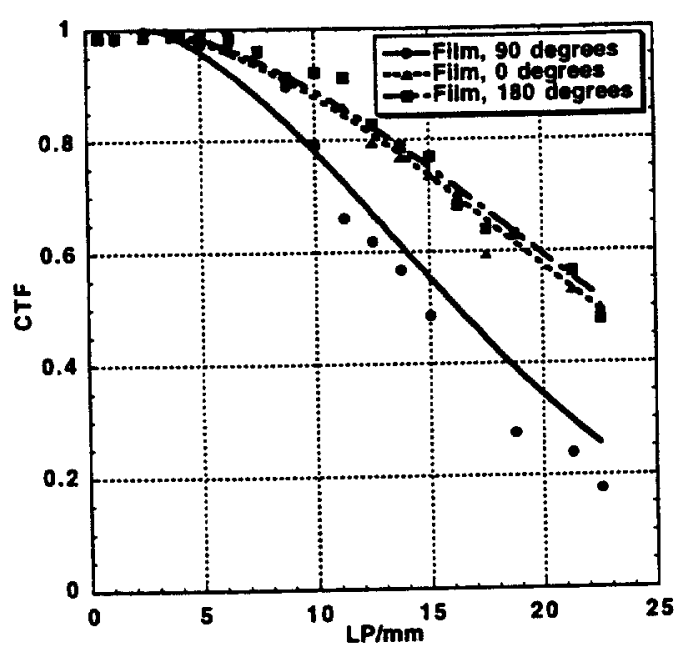

(a)

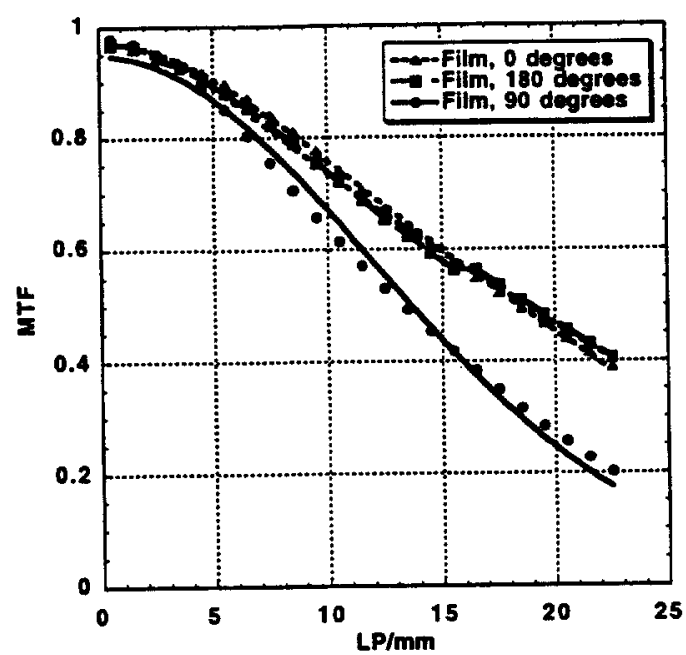

(b)

Figure 7. a) CTF plot and b) MTF plot showing data obtained with film as the recording medium and with the mask at various orientations.

The lower contrast in the $\mathrm{CCD}$ is possibly due to the large pixel size of the current $\mathrm{CCD}$ camera ( $24 \mu \mathrm{m}$ square) and the additional fiber optic bonded directly to the front of the CCD array. The fiber optic reducer degrades the MTF because of reflections at its surfaces and blurring caused by minor misalignment of the fibers.

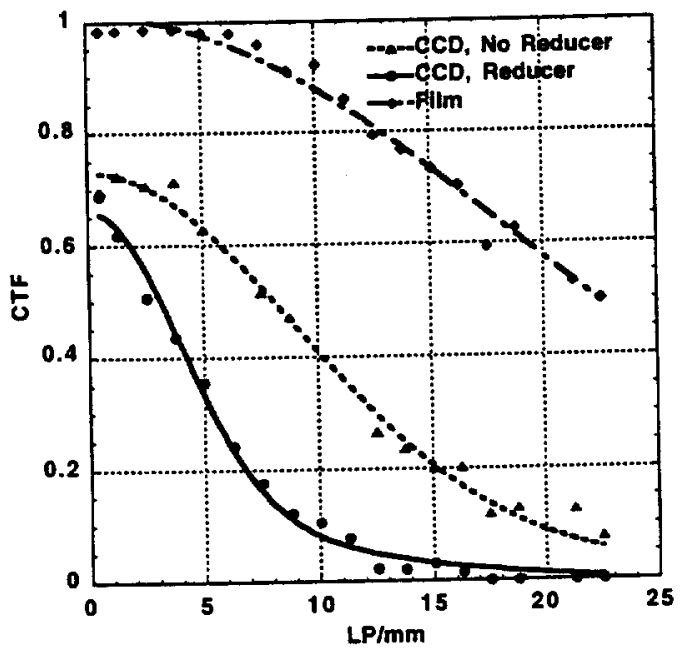

(a)

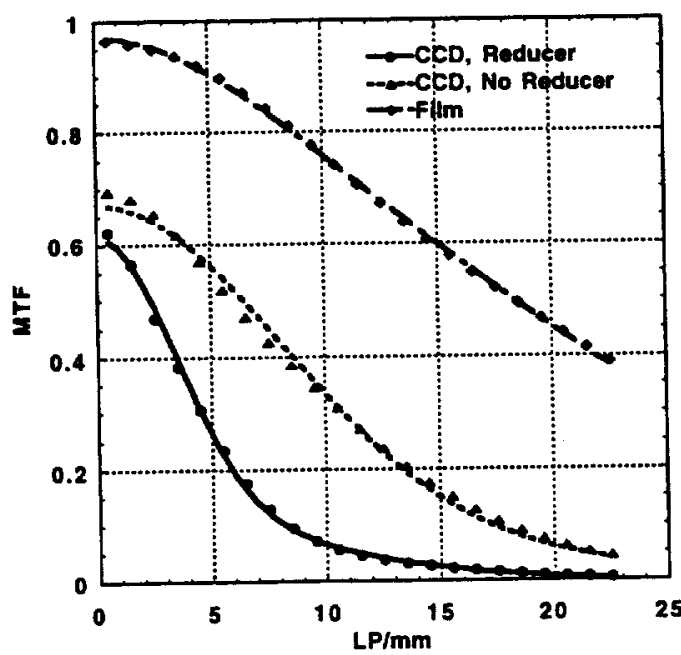

(b)

Figure 8. a) CTF plot and b) MTF plot showing data obtained with film and the CCD camera with and without the fiber optic reducer as the recording medium. 
Table 2 shows the gaussian curve fit formula and coefficients for the experimental data.

Table 2: Double Gaussian Curve Fit Formula \& Coefficients

\begin{tabular}{|c|c|c|c|}
\hline & $\mathrm{a} 0$ & al & a2 \\
\hline Film, $0^{\circ} \quad$ CTF & 18.052 & 0.05286 & 0.05306 \\
\hline Film, $90^{\circ}$ CTF & 12.863 & 0.07303 & 0.07409 \\
\hline Film, $180^{\circ} \mathrm{CTF}$ & 18.657 & 0.04698 & 0.05194 \\
\hline $\mathrm{CCD}$, reducer CTF & 2.1564 & 0.10315 & 0.25868 \\
\hline $\mathrm{CCD}$, no reducer $\mathrm{CTF}$ & 6.1690 & 0.04436 & 0.11138 \\
\hline Film, $0^{\circ} \quad$ MTF & 14.973 & 0.1462 & 0.055025 \\
\hline Film, $90^{\circ} \quad$ MTF & 10.790 & 0.033915 & 0.084621 \\
\hline Film, $180^{\circ}$ MTF & 15.374 & 0.1735 & 0.051915 \\
\hline$\overline{C C D}$, reducer MTF & 1.6683 & 0.11707 & 0.29698 \\
\hline $\mathrm{CCD}$,no reducer MTF & 5.0738 & 0.046122 & 0.12324 \\
\hline
\end{tabular}

The $\mathrm{S} / \mathrm{N}$ ratio using film as the recording medium was 3.4 and that of the CCD was 9.5 . This means that for an equivalent signal level, the film contains 2.8 times as much noise as the CCD.

\section{Conclusions \& Future Work}

In conclusion, we have developed a high quality CTF mask for use on a Nova $\mathrm{x}$ ray imager, and we have used this mask to determine the CTF and MTF using both film and a CCD camera as the recording medium. The MTF plots show that the microchannel plate has a uniform response within our measurement accuracy across its strips; however, it exhibits anisotropy. The MTF plots also indicate that the film has a superior resolution response as compared to the current $C C D$ camera configuration. The higher 
$\mathrm{S} / \mathrm{N}$ ratio measured for the $\mathrm{CCD}$ data is, however, as important as its resolution response, which could be improved by using a smaller pixel-format camera.

In future work, two additional phosphors will be tested: P-11 and an aluminized P-20. It is expected that the film will be more sensitive to the P-11 and the CCD to the P-20 since the CCD responds more sensitively to optical light of longer wavelengths such as those emitted by green-glowing P-20, while film responds more sensitively to light with smaller wavelengths such as those emitted by blue-glowing P-11. The advantage of the aluminized P-20 is to reduce blurring caused by reflections off of the MCP. However, the grain size of $\mathrm{P}-20$ is much larger than that of $\mathrm{P}-11$, resulting in a reduction in resolution. In the future, we anticipate the use of the $C C D$ camera, including its accompanying cooling lines and a newly designed mounting device for coupling the $\mathrm{CCD}$ camera to the phosphor, in Nova laser experiments as an improved replacement for film packages in Nova diagnostics.

\section{Acknowledgments}

The authors would like to acknowledge the contributions of personnel from the Nova laser and target fabrication facilities, mechanical engineering division, and A division. This work was performed under the auspices of the U.S. Department of Energy by the Lawrence Livermore National Laboratory under Contract No. W-7405-Eng-48. 


\section{Appendix A: CTF to MTF Conversion Formula Derivation}

Let $F(k)$ represent the MTF and $F(w)$ represent the CTF.

We know that $F(k)=\operatorname{int}\left(f(x) * e^{-i k x} d x,-\infty, \infty\right)$.

Considering also that $f(x)=F^{-1}(w)$, it follows that $F(k)=$ int $\left(F^{-1}(w)^{*} e^{-i k x} d x,-\infty, \infty\right)$.

$F(w)=\operatorname{int}(f(x) *$ square-wave kernel $d x, 0, \infty)$.

$F^{-1}(w)=f(x)=\operatorname{int}(F(w) *$ square-wave kernel $d w, 0, \infty)$

Like the Fourier kemel is a basis for Fourier space, the square-wave kernel is a basis for square-wave space. Sine waves form a basis for a set of functions. The Fourier transform, int $\left(\mathrm{f}(\mathrm{x})^{*} \mathrm{e}^{-\mathrm{ikx}} \mathrm{dx},-\infty, \infty\right)$, is actually an inner product. By definition, the kernel is orthogonal, such that if one multiplies two square waves of equal frequency and integrates from $-\infty$ to $\infty$, one gets $\infty$; while if the square-waves have different frequencies, one gets 0 . Thus, the square-wave basis is a sum of square-waves. More specifically, it is the Fourier series of a unit square-wave.

So, the square-wave kernel $=4 / \pi * \sum\left[\sin \left[(2 n-1)^{*} w^{*} x\right] /(2 n-1)\right], n=1 \rightarrow \infty$

$$
\begin{aligned}
F(k)= & \operatorname{int}\left(f(x) * e^{-i k x} d x,-\infty, \infty\right) \\
= & \operatorname{int}\left(e^{-i k x} * \operatorname{int}(F(w) * \text { square-wave kernel dw, } 0, \infty) d x,-\infty, \infty\right) \\
= & \operatorname{int}\left(F(w) * \operatorname{int}\left(e^{-i k x} * \text { square-wave kernel } d x,-\infty, \infty\right) d w, 0, \infty\right) \\
= & \operatorname{int}\left(F(w) * \operatorname{int}\left(e^{-i k x} * 4 / \pi * \Sigma\left[\sin \left[(2 n-1)^{*} w^{*} x\right] /(2 n-1)\right], n=1 \rightarrow \infty d x,-\infty, \infty\right)\right. \\
& \quad d w, 0, \infty) \\
F(k)= & \pi / 4 *[F(w)+F(3 w) / 3-F(5 w) / 5+F(7 w) / 7-F(9 w) / 9+\ldots]
\end{aligned}
$$




\title{
Rock Properties \\ for Ground Motion Prediction at \\ Yerba Buena Island
}

Edgar D. Hardy

Supervisor: Brian Bonner

Building 243, Rm. 1014, L-219

Lawrence Livermore National Laboratory University of California

Livermore, California 94551

\begin{abstract}
Tests were done on a rock sample from Yerba Buena Island to test the rock's behavior during earthquakes. These simulation tests were done to aid in research on the prediction of stong ground motion in the Bay Bridge area. As a result we found that the rock sample's shear modulous (stiffnes) was much higher when partially saturated with water than when completely dry in eathquake simulated conditions. We also found that the rock contained micro fractures, which were closed by application of uniaxial stress.
\end{abstract}

\section{Introduction}

Earthquakes have been one of the leading natural hazards in Califomia and particularly in the Bay Area where the disastrous Loma Prieta earthquake of 1989 occurred. This phenomenon has cost the Bay Area many human lives and millions of dollars in damage to buildings, bridges, and other structures. Scientists have researched the 
effects of previous earthquakes in order to predict the time, general area, and intensity of the next one that may occur.

\section{Background}

One of the biggest projects in the Bay area right now is the rebuilding of the east span of the Bay Bridge. Seismologists, structural and soil engineers, geophysicists, and other scientists are working with Caltrans (California Transportation) in order to build a safer, more flexible bridge that will be able to withstand the effects of a highintensity earthquake. (Richter magnitude of $\sim 7.5$ on the Hayward fault).

Our project is a part of the geophysics and seismology research contributing to the overall Bay Bridge research. In our project we took rock samples supplied by Caltrans from Yerba Buena Island (the natural anchor for the Bay Bridge) and subjected them to earthquake conditions using a torsion oscillator. We measured and calculated such properties of the rock samples as strain, stress, and shear modulous (stiffness) under these conditions.

Using measurements of the strain dependence of rock properties we can understand the behavior of the rock during an earthquake and hopefully predict how it will behave during the next earthquake. 


\section{Apparatus}

The testing apparatus we used to carry out our experiments was a torsional oscillorator (the torque-Q) designed by Brian Bonner ( $\mathrm{a}$ geophysicist at Lawrence Livermore National Laboratory). The torque$\mathrm{Q}$ uses an electromagnetic assembly powered by sinusoidal voltages which applies an abitrary torque to a cylindrical sample by driving a permanent magnet connected to the sample (for more details see Bonner and Wannamaker, 1991). The machine also includes proximity detectors that measure displacements of the arms extending from the torsion rods which twist the sample. Torque is proportional to the deformation of aluminum that can be measured by the proximity detectors located at the fixed end of the apparatus to the right of the sample (refer to figure ). Strain is proportional to the twist of the sample measured by the proximity detectors located to the left of the sample ( refer to figure ). From the ratio of the torque to the strain, we can calculate the shear modulus of the sample. We can also apply a uniaxial stress to the sample along the symmetry axis using the static end load device. This is a feature of the machine that we use to simulate conditions for the rock sample being at different depths in the earth. 


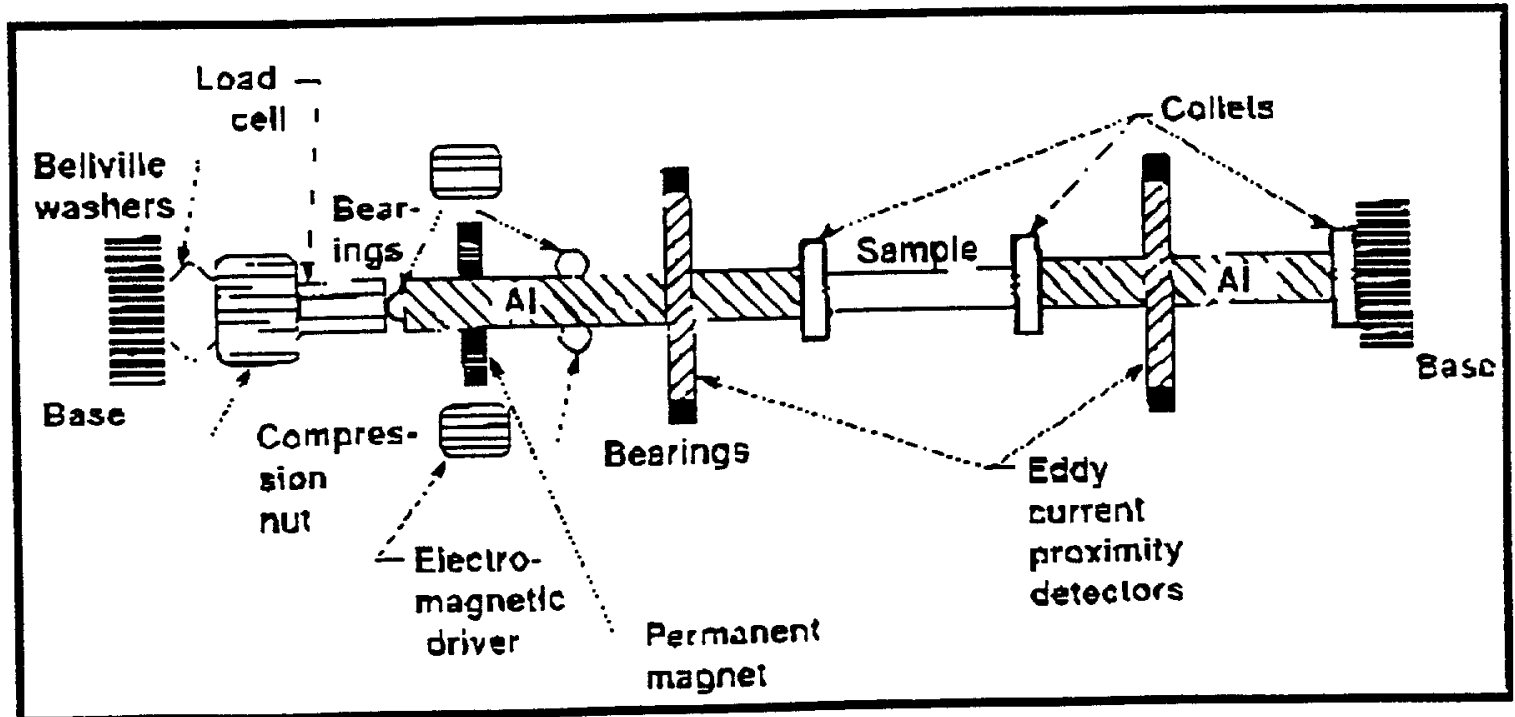

\section{Procedure}

For each experimental run we performed a series of tasks.

Samples of .351 in diameter and 2 in long were prepared and tested by applying a pre-twist by inputting a DC voltage to the magnets. Each experiment was categorized as a strain sweep run or a frequency sweep run. In the frequency sweep run, we varied the frequency (usually in the frequency range of earthquakes) while applying two constant levels of torque. In the strain sweep run, we applied various torques to the sample while maintaining a constant frequency. We performed each experimental run at room humidity, one hundred percent humidity, and also with the sample dried with nitrogen. We applied a uniaxial load during some strain sweeps as discussed above. 


\section{Data Reduction and Analysis}

After each test the results were sent via a program made in LabView to a spread sheet in Kaleidagraph. From there we used the raw data such as phase which is the measure of twist from the proximity detectors and amplitude which is the measure of torque in voltage form. (In this project we only analyzed the data from the strains

sweeps.) We then created two new simple programs in Kaliedagraph to calculate rock properties such as the shear modulus, shear strain, and attenuation of the sample and then label their respective columns on the particular spread sheet. Both programs were labor saving and time saving.

\section{Results}

After extensive testing we observed very unusual behavior from the Yerba Buena Rock. We noticed that the shear modulus of the rock during the earthquake conditions was significantly higher when the rock was partially saturated with water than when it was completely dry. The shear modulus increased from 14 to $18 \mathrm{GPa}$ at a strain of $1.0 \mathrm{X}$ $10^{-5}$ when partially saturated. We also saw that the rock sample became stiffer when we applied more end load. This suggested that there were micro fractures in the rock that caused it to have a low shear modulus. As we increased the end load, the micro fractures closed and the rock became stiffer. 


\section{Conclusion}

In conclusion, the end results showed that our experiments were successful. We made a new discovery regarding the behavior of the Yerba Buena rock during strong ground motion. The rock seems to be stiffer when wet. In previous observations of similar rocks, partial saturation caused the shear modulus to drop as the result of chemical softening.

We plan to run more tests in the near future on the same rock, but in this case including an artificial fracture, to determine how much the shear modulus is reduced. We will also test other samples from Yerba Buena Island taken from different depths to see if the same odd behavior occurs.

\section{References}

Bonner, B. P., and B. J. Wanamaker, Nonlinear attenuation effects outside the zone of macroscopic failure, Explosion Source Phenomenology, Geophysical Monograph 65, Plenum Press, New York, 1991. 
EXPRESSION OF DNA REPAIR PROTEIN XPA IN A BACTERIAL HOST

\author{
Eric D. Hempel \\ Supervisor: Rod Balhorn \\ Building 361, Room 1342, L-452 \\ Lawrence Livermore National Laboratory \\ Livermore, CA 94550
}

Nucleotide excision repair (NER) is a repair system capable of eliminating a wide variety of DNA damage. The importance of NER has been clearly demonstrated by studies of the human genetic disease xeroderma pigmentosum (XP). This disease is characterized by hypersensitivity of skin to UV-irradiation especially sunlight, a high incidence of skin cancer tumors and neurological disorders (Cleaver and Kraemer 1989).

Cells from XP patients are sensitive to death by UV-irradiation and have a defect in the NER process. It has been shown by other research groups that the protein xeroderma pigmentosum group A (XPA) binds preferentially to UV-, cisplatin-, and tetroxide- damaged DNA, showing that XPA is involved in the recognition process of NER (Jones and Wood 1993). The basis of my project was to develop protocol for the overexpression of XPA in an E. coli host and it's purification to near homogeneity for further study and analysis.

The human DNA construct for the XPA protein that I used was obtained from Lei Li at the University of Texas Medical Center, MD Anderson Cancer Center. AT7 expression system was used to produce the XPA proteins. The pET-14b/XPA plasmid that was obtained from Lei Li was transformed into the $E$. coli Strain BL21 (DE3): this strain utilizes the $\Pi 7$ expression system. Transformed bacteria were grown at $37^{\circ} \mathrm{C}$ to mid-log phase, optical density 
A600 of $0.6-1.0$, and isopropyl-1-thio- $\beta$-D-galactopyranoside (IPTG) was added to a final concentration of $1 \mathrm{mM}$. This chemical induced the bacterial T7 expression system to produce the target XPA protein over and over again. The procedure was optimized to give the largest possible amount of protein in the shortest amount of time. After 3 hours of expression with IPTG the cell culture were pelleted by centrifugation at $6000 \times \mathrm{xg}$ for $20 \mathrm{~min}$., pellets were frozen at $20^{\circ} \mathrm{C}$ overnight.

Purification was the next step in my project and this was performed by utilizing the pET-14b plasmid which adds to the end of the XPA protein a series of six histidine amino acids called the "His-Tag". This "His-Tag" sequence allowed for the easy, first step of purification of the XPA protein on a column containing immobilized nickel ions. XPA protein with the "His-Tag" has an affinity for the nickel ion and binds to it, while most of the bacterial proteins flow through the column without binding to the nickel. The protein can then be eluted off the column with a buffer containing imidazole. Fractions were collected and checked for purity on 15\% SDS-PAGE and Coomassie stained. Purity from the nickel column was qualitatively assessed to be $80-90 \%$.

Greater purity was required so the fractions from the nickel column were pooled and then concentrated with "Slide-A-Lyzer" concentrating solution (Peirce) to $\sim 5 \mathrm{mg} / \mathrm{ml}$. The concentrated XPA samples were then passed through a gel filtration column chromatography (GPC) which allowed for the separation of molecules based on size. Samples were collected from the GPC column and again were qualitatively checked on 15\% SDS-PAGE final purity was approximated to be greater than $98 \%$. The final yield of pure XPA protein after both steps of purification was $\sim 6 \mathrm{mg}$ per $500 \mathrm{ml}$ culture of cells. All purification steps were performed in denaturing conditions so purified protein 
needs to be refolded to its native confirmation. This was done by dialyzing the protein for 16 hours against a tris buffer with reducing agent.

The results were greater than expected and produced large amounts of human XPA protein that will be studied in the future. Future studies included producing crystals from the XPA protein so that it can be studied by X-ray Crystallography. This process will allow researchers to find the 3 dimensional structure of the XPA protein. Knowing the structure of such proteins will lead to a better understanding of the process of nucleotide excision repair and possibly other forms of human cancer.

Cleaver, J.E. and K.H. Kraemer, "Xeroderma Pigmentosum, in: C.R. Scriver et al. (Eds.), The Metabolic Basis of Inherited Disease, 6th Edn., Vol. 2, McGraw-Hill, New York 1989, pp. 2949 - 2971.

Jones, J.S. and R.D. Wood, "Perferential Binding of the Xeroderma Pigmentosum Group A Complementing Protein to Damaged DNA, Biochemistry, Vol. 32 , 12096-12104. 


\title{
CONSTRUCTION OF A COMPUTER MODEL FOR LINEAR ANALYSIS OF BIXBY CREEK BRIDGE
}

\author{
Matthew S. Hoehler \\ Supervisor: David McCallen \\ Building 131; Room 1171; L-126 \\ Lawrence Livermore National Laboratory \\ Livermore, California 94550
}

\begin{abstract}
The construction of a computer model of Bixby Creek Bridge generated for analysis using linear finite element methods is documented. Assumptions and simplifications used for the modeling are presented and discussed. An eigenvalue analysis is carried out for the first thirty five modes of the structure and the critical frequencies and mode shapes are presented.
\end{abstract}

\section{INTRODUCTION}

In the past few years the state of California has engaged in an all out effort to safeguard its critical infrastructure from seismic hazards (i.e. earthquakes). This report documents one component of a study to be performed by Lawrence Livermore National Laboratory (LLNL) for the California Department of Transportation (Caltrans). The study will investigate the effects of earthquakes on deck-type arch bridges with the intent to characterize the behavior of these structures during seismic events. Structure behavior characterization allows for the development of adequate seismic retrofit measures. The scope of the LLNL study includes performing linear and nonlinear earthquake analysis of 
the Bixby Creek Bridge incorporating the empirical Green's functions method [Ref 1] to generate realistic, site-specific ground motion.

The Bixby Creek Bridge is a large, concrete deck-type arch bridge located about 18 miles south of Carmel on the California coast (See Location Map). The bridge, completed in 1932 , is one of several concrete arch bridges built along the California coast during the 1930's. The Bixby Creek Bridge is located along a spectacular section of coastline and spans a very deep canyon.

This setting, and the inherent graceful-

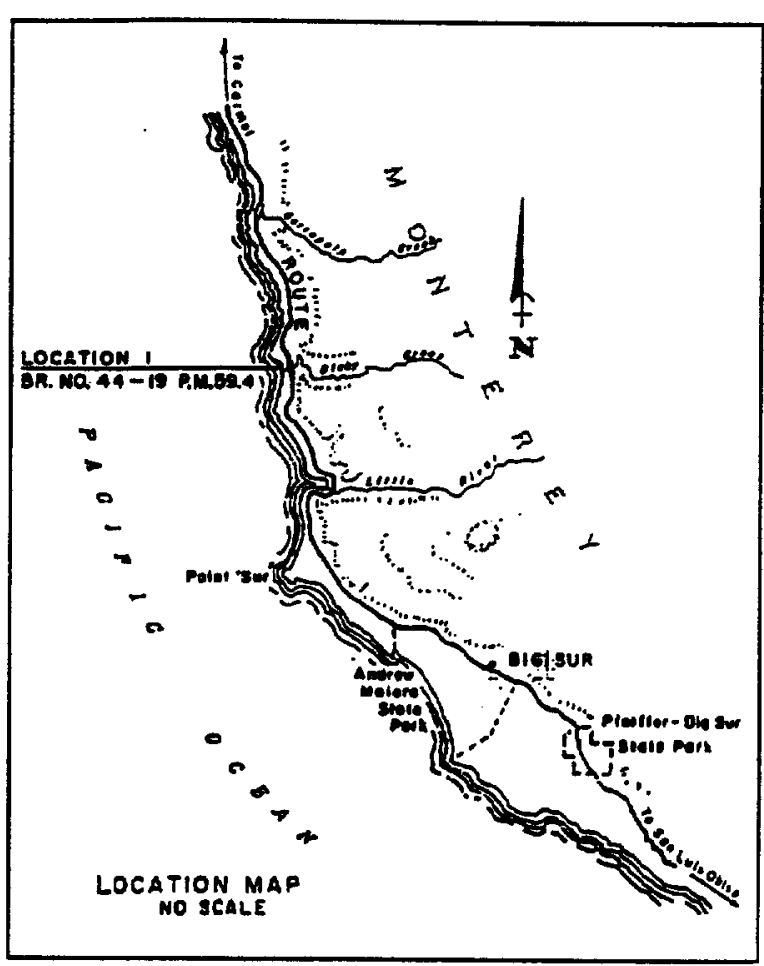

Location Map [Ref 2] ness of the structure, makes Bixby Creek Bridge one of the most picturesque bridges in the world.

The Bixby Creek Bridge was selected for this study primarily because it is representative of the entire class of deck-type arch bridges. The results obtained from this study can therefore will be applicable to other similar bridges. The large arch span and the geologic setting of the Bixby Creek Bridge provided additional motivation for the selection of this site. The total length of the bridge, measured along the centerline, is 714 feet. With the North and South approaches removed, the main arch-span is 320 feet. The arch-ribs have a vertical rise of approximately 120 feet [Ref 3]. These dimensions make the Bixby Creek Bridge one of the largest bridges of this style. The geologic setting of the bridge will pro- 
vide LLNL seismologists with the opportunity to study earthquake wave propagation across deep V-shaped canyons. One of the key components of this study will be the application of the ground motions obtained from the seismologists' investigations and assessing the effects of differential ground motion on deck-type arch bridges.

This report focuses on the generation of the linear model used in the LLNL study. The results presented herein will be used to determine the characteristic natural frequencies of the structure and to perform a linear analysis of the structure. The linear analysis will allow for comparison with the fully nonlinear model once the nonlinear model has been constructed. The results of these models will be verified in subsequent reports once the actual structure has been instrumented with acceleration measuring devices.

The modeling section of this report presents a condensed version of the modeling assumptions and simplifications used for the construction of the model. Additional details and diagrams can be found in a companion report [Ref 4]. The generation of the finite element mesh, some assumptions made to account for nonlinearites, and the boundary conditions of the structure are presented in the modeling section.

The results obtained from an eigenvalue analysis of the first 35 modes of the structure are presented in the results section. The analysis shows that for these modes the structure has natural periods of vibration varying from 1.082 to 0.165 seconds; these periods are for the 1st and 35th modes respectively. Mode shapes for the modes with the largest modal mass participation in the global $\mathrm{X}, \mathrm{Y}$, and $\mathrm{Z}$ directions are shown.

Finally, the implications of the results are discussed in the context of future analysis to be performed on the structure. 


\section{Modeling}

The computer model of the Bixby Creek Bridge was created using the SLIC [Ref 5] mesh generator. SLIC is an interactive, graphic mesh generator for finite-element and finite-difference application programs. SLIC was created at LLNL for use with various finite elements analysis programs. The SLIC output files produced for this model were formatted for the GEMINI [Ref 6] finite element code. All of the dimensions used to construct the model were obtained from either the original plans for the bridge (1931) [Ref 3] or from the proposed retrofit plans (1996) [Ref 7].

Bixby Creek Bridge is composed of three structural sections, namely the South Approach Span (Spans 1, 2, 3), the Main Arch Span (Span 4), and the North Approach Span (Spans 5, 6, 7, 8, 9, 10) (See FIGURE 1.). A global coordinate system for the model was selected such that the origin is located at the south-east corner of the bridge, at an elevation coincident with the lowest point of the south abutment (See FIGURE 2.).

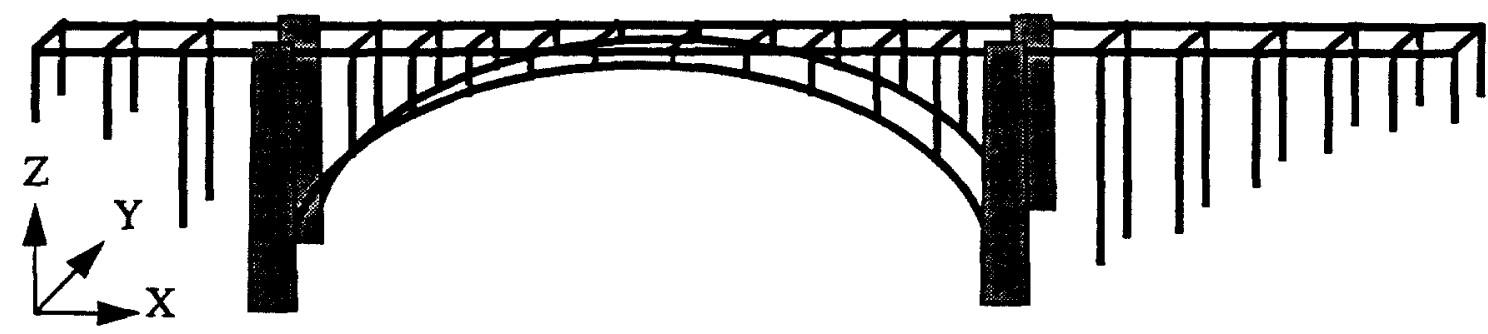

FIGURE 2. Sketch of Global Coordinate System

SLIC requires that all critical geometry points be denoted by both a physical and a logic space coordinate. A record of these points and their numbering systems can be found in Reference 4. Some elevations were not legible in the original bridge plans. In these cases, a linear interpolation between the two bounding elevations was used to determine the unknown elevations. 


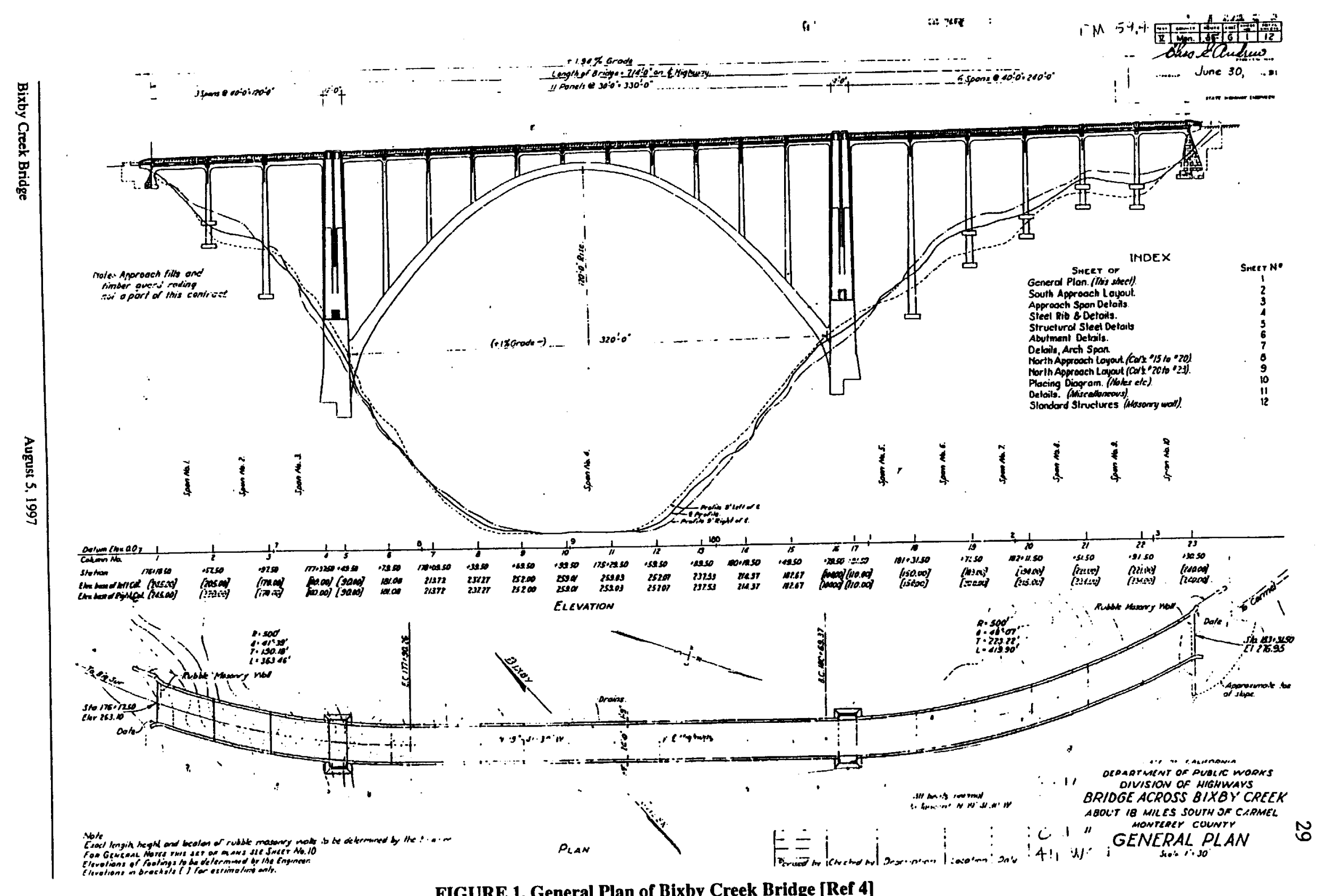

FIGURE 1. General Plan of Bixby Creek Bridge [Ref 4] 
The retrofit bridge plans give elevations for the bases of the columns of the north and south abutments. If one looks at the plans for the bridge, however, one will see that most of these footings are located between 5 and 25 feet below the ground surface (See FIGURE 1.). It was assumed for the model that the length of column located below the ground surface was surrounded by fill and/or incompetent rock (See FIGURE 3.). The elevations given in the plans were therefore assumed to be the fixed point at the base of the approach columns. The thickness of the footing ( 2.5 feet) was subtracted from the total length of the column.

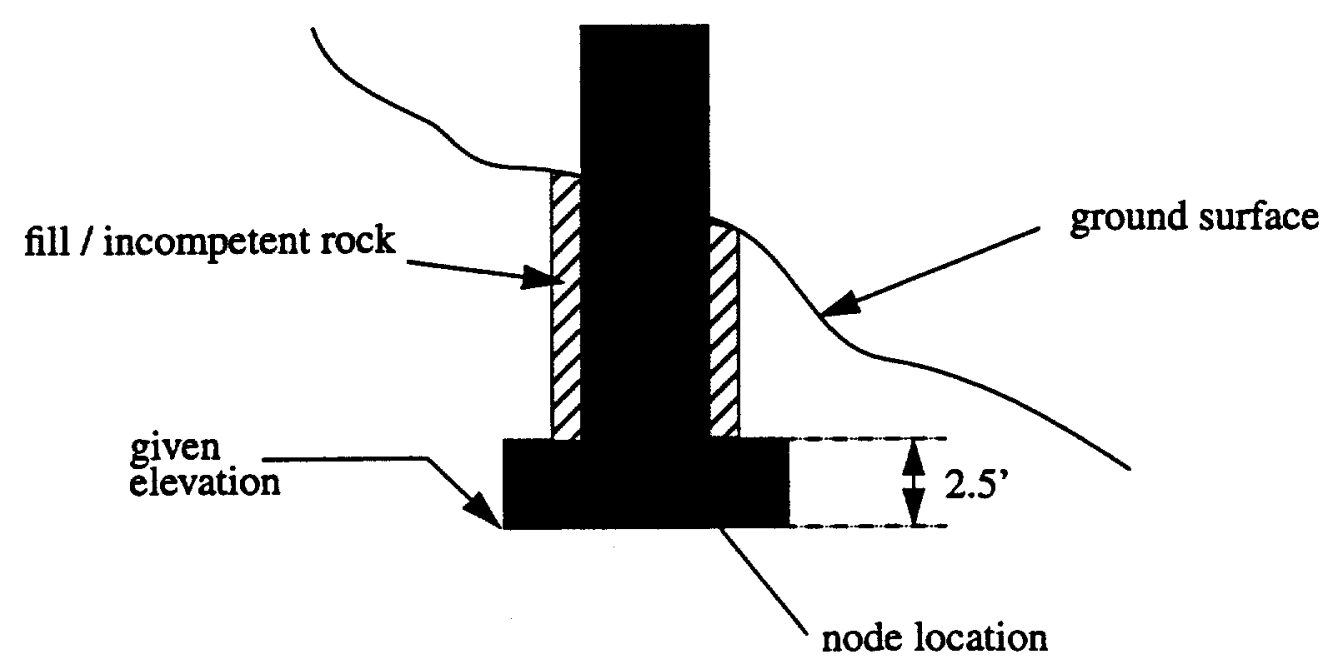

FIGURE 3. Approach Column Footing

A number of assumptions were made to model the north and south towers of the bridge. A record of all of these assumptions can be found in reference 4 . The two most significant points are mentioned here. First, it was necessary to develop a scheme to couple rotations from the shell and beam elements (these comprise the majority of the tower) to the solid elements (comprises lower 1/5th of tower) (See FIGURE 4.). Coupling was required because the nodes for solid elements only track translational motion. The coupling was achieved by extending the beam and shell elements into the solid elements at locations where there were interfaces. 


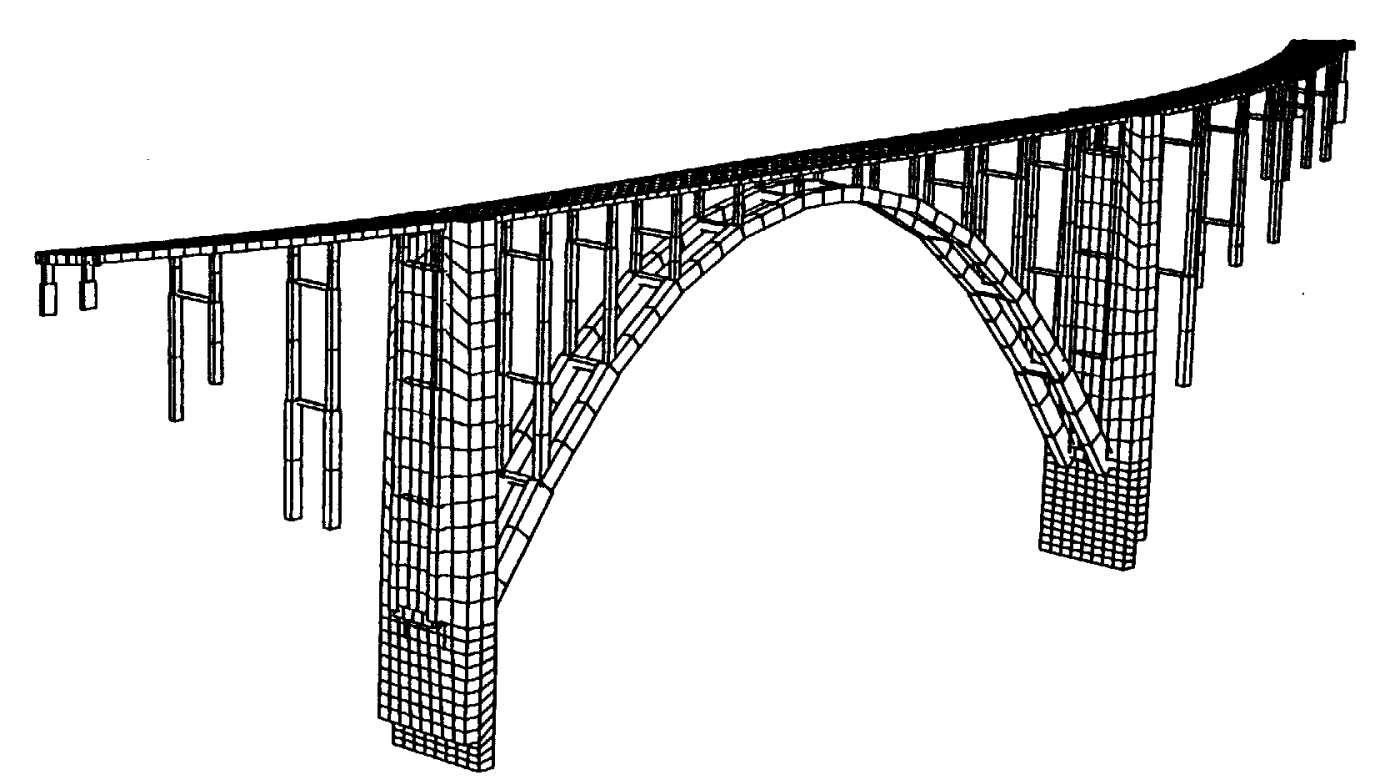

FIGURE 4. Finite Element Model of Bixby Creek Bridge 
The second significant modeling assumption made for the towers, and probably the most critical assumption made for the model, was the load transfer path from the deck beams to the tower. It appeared from the plans that the only connectivity between the deck and the tower is provided by the connection of the longitudinal deck beams to the tower columns (i.e. there are no connections between the deck and the tower shells) (See FIGURE 5.). Unfortunately, there were no clear and explicit details of the connections at tops of the towers. For this model it was assumed that all load transfer from the deck to the tower occurs through the tower columns.

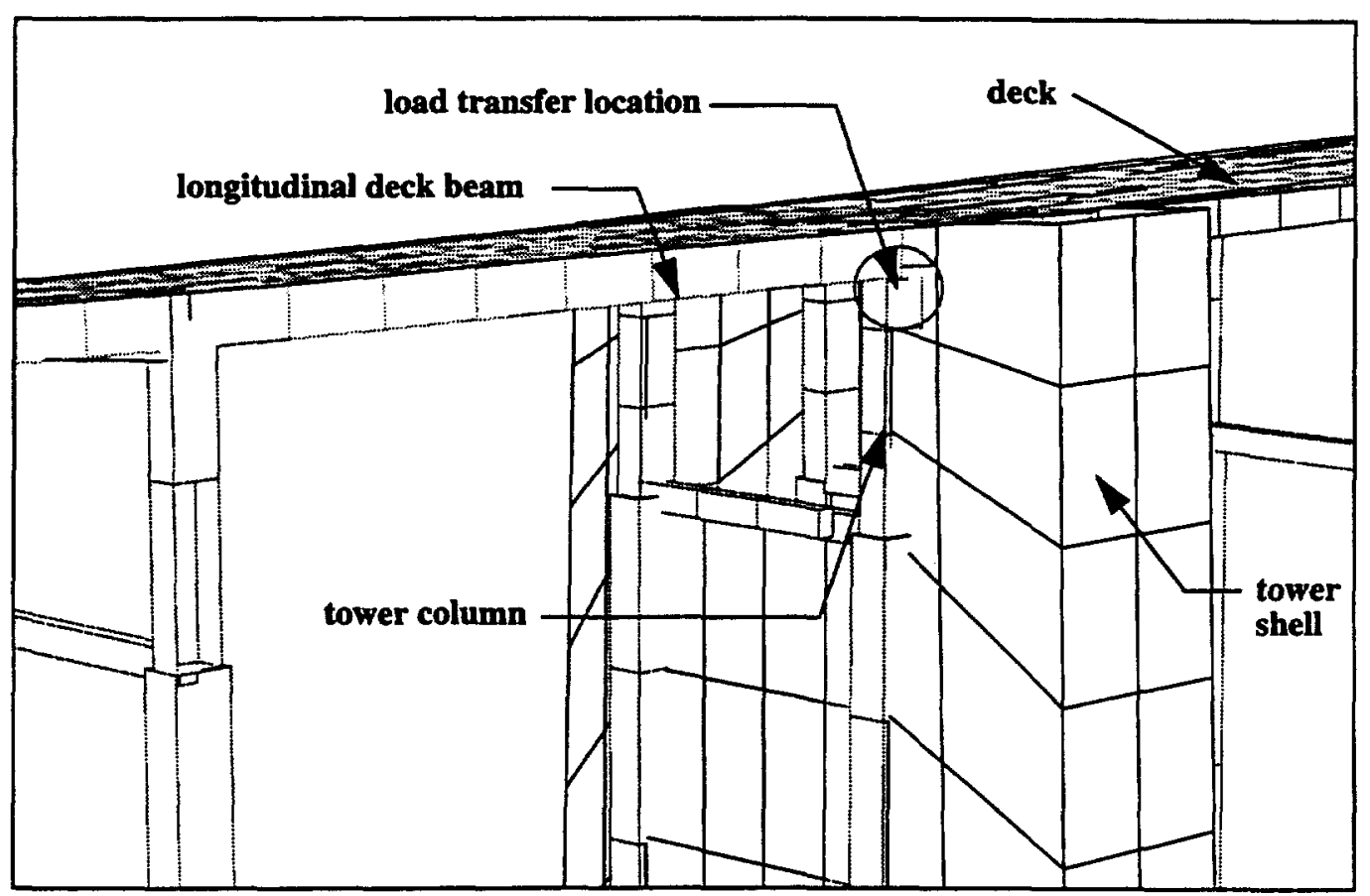

FIGURE 5. Column / Tower Connections

The entire load transfer during dynamic, and static, loading is therefore occurring across four 1.5 foot by 2 foot columns. If this is indeed the correct connection detail, it would be a critical location for earthquake retrofit measures. These connections will be reassessed after the actual bridge has been inspected in the field.

For this model all expansion joints were ignored. This was judged reasonable because of a retrofit of the bridge in which the expansion joints were restrained by steel 
bands [Ref 2] (See FIGURE 6.). As Figure 6 shows, these bands will only restrict expansion of the joint, compression is still possible. When a nonlinear model is constructed, it will be necessary to take a closer look at these expansion joints and refine this assumption.

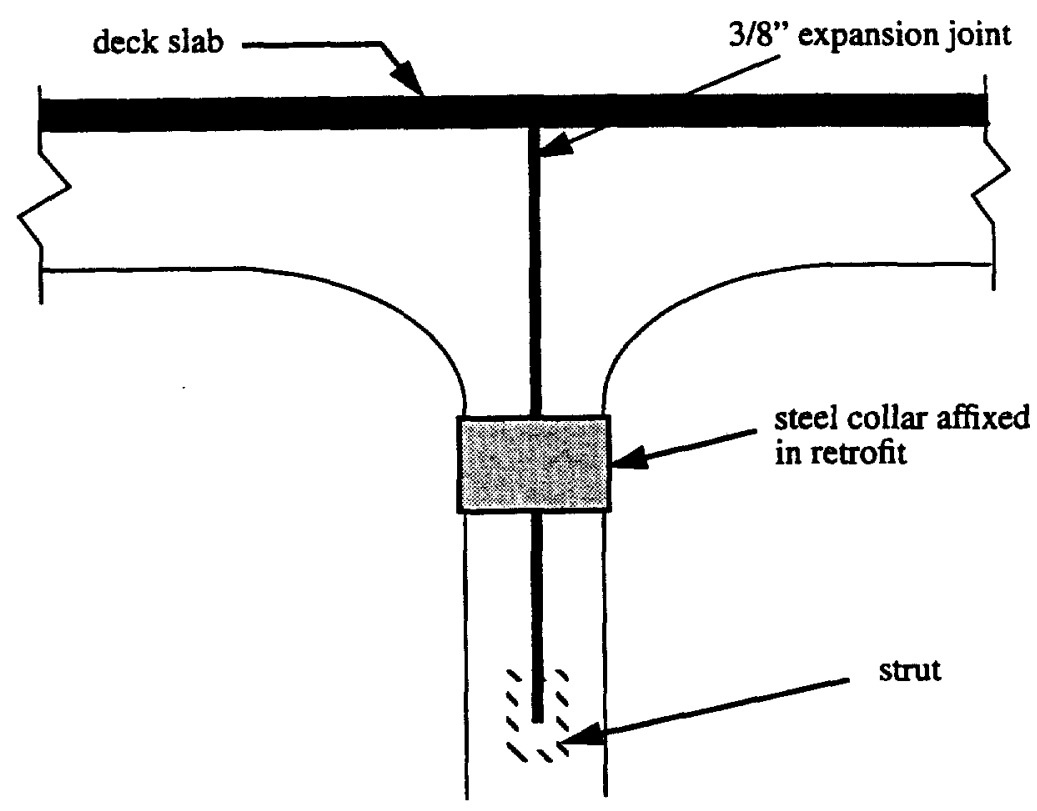

FIGURE 6. Expansion Joint Detail

The final modeling note of significance is the calculation of the torsional moment of inertia $(J)$ for the hollow arch columns. There are three columns that are rectangular beam elements with hollow centers (geometric property \#'s 10, 11,43) [Ref 4]. The sections are located in the two longest pairs of columns in the main arch (See FIGURE 7.).

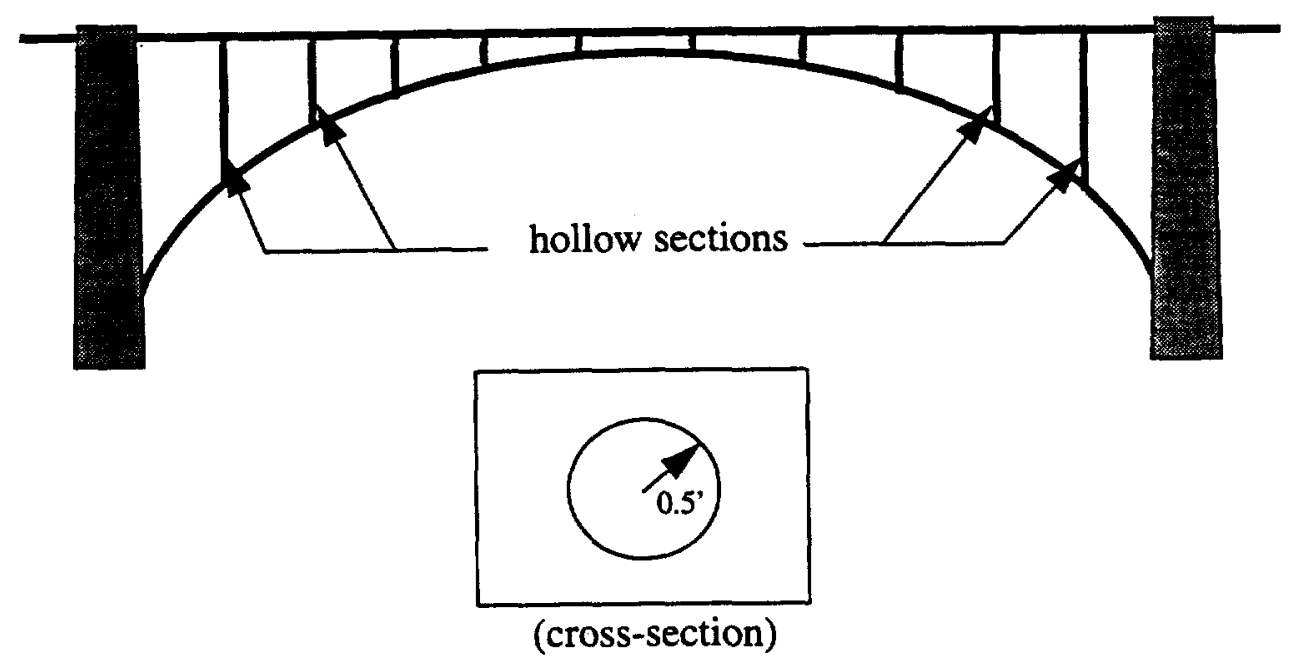

FIGURE 7. Hollow Column Location 
Rather than trying to calculate an analytic solution for the torsional inertia, which would be quite difficult, numerical solutions were found by modeling the sections with solid elements and performing finite element analysis using the GEMINI code.

The first step in finding an empirical solution was to create the solid model using SLIC. The mesh and corresponding logic coordinates are shown (See FIGURE 8.). After

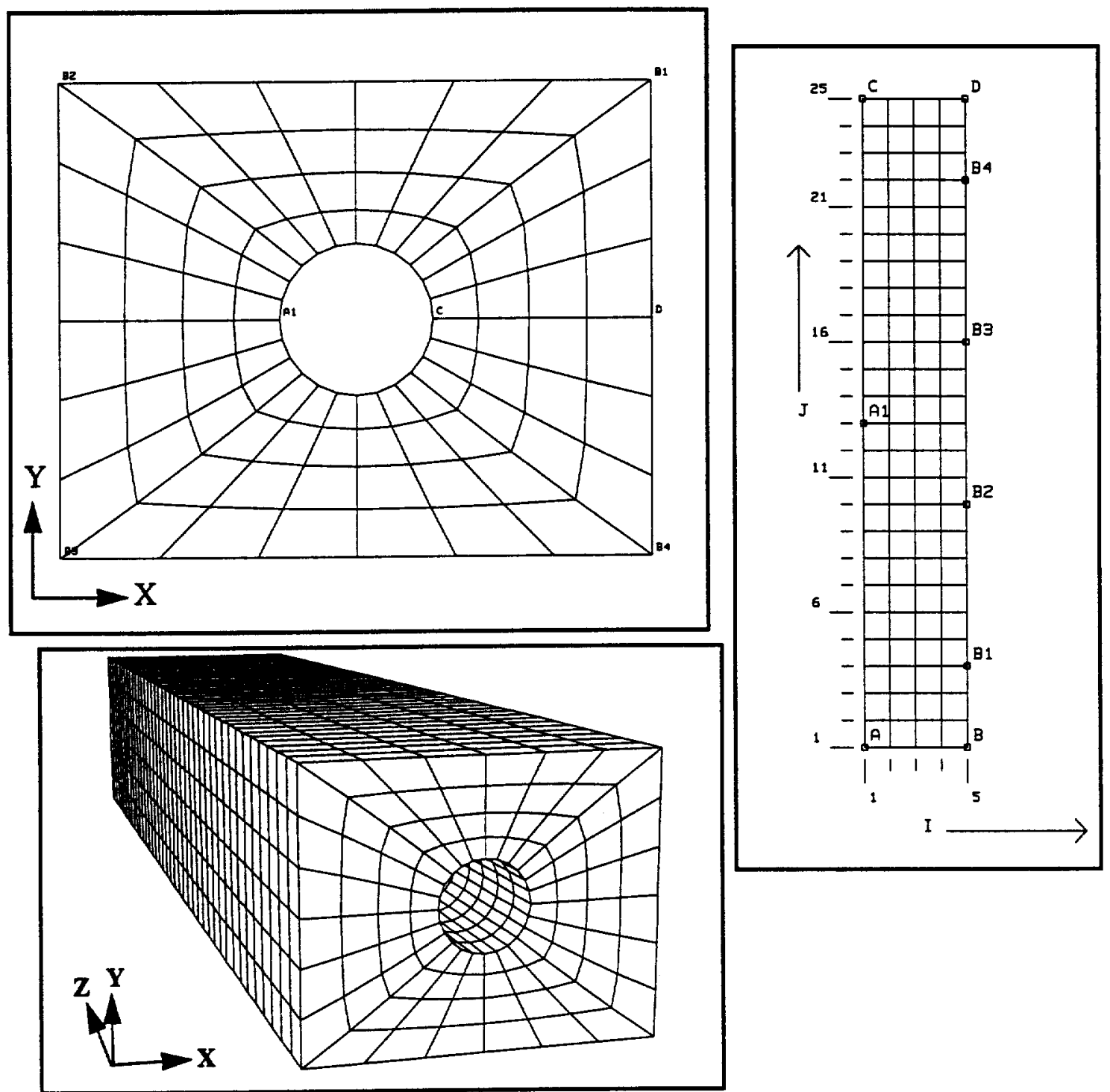

FIGURE 8. Finite Element Mesh \& Logic Coordinates

the model was created, one end was fixed and at the other end four equal point loads were 
applied (See FIGURE 9.). Using the material properties of actual beams on the bridge, a rotational displacement was numerically calculated for the sections (See FIGURE 10.).

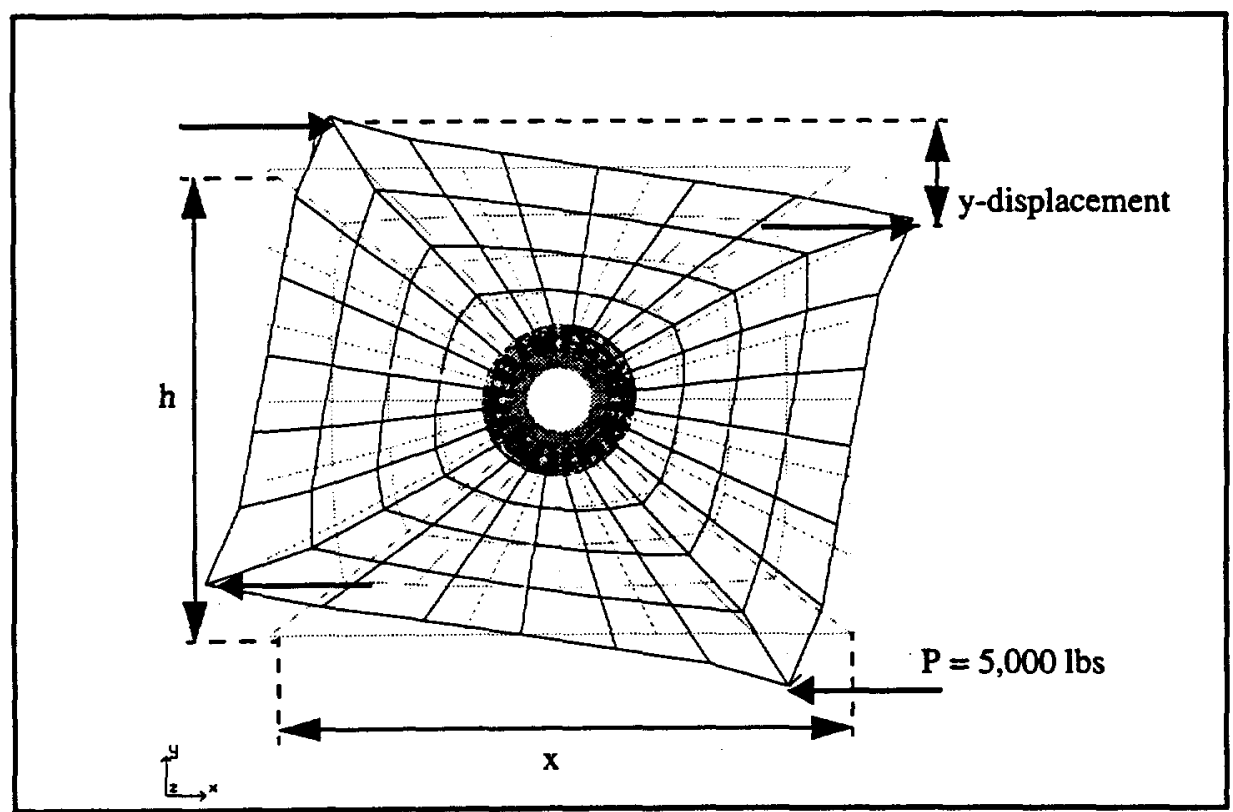

FIGURE 9. Beam Torsion

$$
\theta=\frac{T L}{G J} \Rightarrow J=\frac{T L}{G \theta}
$$

where:

$$
\begin{array}{ll}
\theta=\frac{(y-\text { displacement })}{x} & \begin{array}{l}
E=6.355 \times 10^{8} p s f \\
v=0.175
\end{array} \\
G=\frac{E}{2(1+v)} & \text { Notes- }
\end{array}
$$

Notes-
length used is $1 / 2$ of the total length of the beam.
y-displacement is found numerically.
the small changes which occur in $x \& \mathrm{~h}$ after defor-
mation are ignored.
E \& $v$ are material properties

FIGURE 10. Equations used to Determine Torsional Inertia

The required discritization for the mesh was determined by successive solutions with finer and finer mesh sizes until the difference between solutions for $\mathrm{J}$ converged to 
within $5 \%$ of the total value of J. This was done for a solid (i.e. no hole) section with outside dimensions that were the same as those of geometric property number 10 (GPS 10). Any cross-section could have been used to determine the required mesh discritization, however, by using a section similar to GPS 10 , one could compare the results of the numerical solution with an approximate analytic solution for a solid section. Adequate convergence occurred at a mesh density of $6 \times 6$. This mesh density was then applied to the hollow sections.

Table 1 shows the $\mathrm{J}$ values obtained during the iterations. One test was performed to check the adequacy of the length of the test member. Changing the member length from 20 feet to 30 feet only slightly decreased the value of J. One should note that all of the numerically calculated solutions are slightly larger than the approximate analytic solution.

TABLE 1. Iterative Solutions for $J$ for a Solid Beam

\begin{tabular}{|c|c|c|c|c|}
\hline mesh density & $\begin{array}{c}\text { y-disp } \\
(\mathbf{f t})\end{array}$ & $\begin{array}{c}\mathbf{J} \\
\left(\mathbf{f t}^{4}\right)\end{array}$ & change & $\begin{array}{c}\text { convergence } \\
(\%)\end{array}$ \\
\hline \hline $4 \mathrm{X} 4\left(20^{\prime}\right)$ & $1.047 \mathrm{E}-4$ & 21.31 & - & - \\
\hline $4 \mathrm{X}\left(30^{\prime}\right)$ & $1.575 \mathrm{E}-4$ & 21.25 & 0.06 & 0.3 \\
\hline $6 \mathrm{X6}\left(20^{\prime}\right)$ & $1.075 \mathrm{E}-4$ & 20.75 & 0.56 & 2.6 \\
\hline $8 \times 8\left(20^{\prime}\right)$ & $1.086 \mathrm{E}-4$ & 20.54 & 0.21 & 1.0 \\
\hline $\begin{array}{c}\text { approximate } \\
\text { analytical solution }\end{array}$ & & 19.98 & & \\
\hline
\end{tabular}

One should note that the $y$-displacements were taken at a location half-way along the length of the beam, well away from where the point loads were applied. There are likely to be some exaggerated displacements near the point loads. By checking displacements half-way along the length of the beam, the point effects should be negligible. 
Table 2 shows the section properties and constants used for the three hollow-core columns.

TABLE 2. Hollow-Core Column Section Properties

\begin{tabular}{|c|c|c|c|c|c|c|c|}
\hline $\begin{array}{c}\text { Geometric } \\
\text { Property } \\
\#\end{array}$ & $\begin{array}{c}\text { section } \\
\text { length } \\
(\mathbf{f t})\end{array}$ & $\begin{array}{c}\text { 2-dim } \\
\text { ave. } \\
(\mathbf{f t})\end{array}$ & $\begin{array}{c}\text { 3-dim } \\
\text { ave. } \\
(\mathbf{f t})\end{array}$ & $\begin{array}{c}\text { Axial } \\
\text { area } \\
\left(\mathbf{f t}^{2}\right)\end{array}$ & $\begin{array}{c}\mathbf{J} \\
\left(\mathbf{f t}^{4}\right)\end{array}$ & $\begin{array}{c}\mathbf{I}_{2} \\
\left(\mathbf{f t}^{4}\right)\end{array}$ & $\begin{array}{c}\mathbf{I}_{3} \\
\left(\mathbf{f t}^{4}\right)\end{array}$ \\
\hline \hline 10 & 33.53 & 3.12 & 3.87 & 12.07 & 20.44 & 15.02 & 9.75 \\
\hline 11 & 39.94 & 2.35 & 2.94 & 6.91 & 6.59 & 4.93 & 3.13 \\
\hline 43 & 40.00 & 2.35 & 3.10 & 7.29 & 7.25 & 5.79 & 3.30 \\
\hline
\end{tabular}

\section{RESULTS}

The primary goal of this phase of the study was to generate a finite element model of the Bixby Creek Bridge to be used for linear analysis. For this reason, the bulk of this report is contained in the modeling section. In the future, when earthquake motion is applied to the structure, a plethora of data will be obtained which will aid in the determination of adequate seismic retrofit measures. Even without inputting earthquake motion, however, the natural frequencies of the structure can be determined by eigenvalue analysis. The most significant results from an eigenvalue analysis of the first 35 modes of the structure are presented below.

The structure was found to have a natural period of 1.082 seconds in the first mode. In the 35 th mode, the period was 0.165 seconds. The rest of the natural frequencies can be found in Reference 4.

The five most significant modes in the global $\mathrm{X}, \mathrm{Y}$, and $\mathrm{Z}$ directions are given in Tables 3,4 , and 5 . The importance of a given mode was determined by calculating the fraction of the total mass of the structure participating in displacing the structure in a given direction for a given mode (i.e. the more mass that is moving, the more important the 
mode). Pictures of the most important mode shape for movement in a given direction are shown (See Figures 11., 12., 13.).

TABLE 3. Importance of mode in global X-direction

\begin{tabular}{|c|c|}
\hline Importance & $\begin{array}{c}\text { Mode } \\
\text { number }\end{array}$ \\
\hline \hline 1 & 4 \\
\hline 2 & 11 \\
\hline 3 & 8 \\
\hline 4 & 18 \\
\hline 5 & 24 \\
\hline
\end{tabular}

TABLE 4. Importance of mode in global Y-direction

\begin{tabular}{|c|c|}
\hline Importance & $\begin{array}{c}\text { Mode } \\
\text { number }\end{array}$ \\
\hline \hline 1 & 1 \\
\hline 2 & 12 \\
\hline 3 & 7 \\
\hline 4 & 9 \\
\hline 5 & 16 \\
\hline
\end{tabular}

TABLE 5. Importance of mode in global Z- direction

\begin{tabular}{|c|c|}
\hline Importance & $\begin{array}{c}\text { Mode } \\
\text { number }\end{array}$ \\
\hline \hline 1 & 23 \\
\hline 2 & 5 \\
\hline 3 & 28 \\
\hline 4 & 16 \\
\hline 5 & 30 \\
\hline
\end{tabular}

\section{DISCUSSION}

The results presented above will applied to future portions of the study in primarily three ways.

In order to verify the results of both the linear and nonlinear (not yet created) models, the Bixby Creek Bridge will be outfitted with arrays of accelerometers and then vibrated (using a vibration generator) by a harmonic input motion. The response of the 


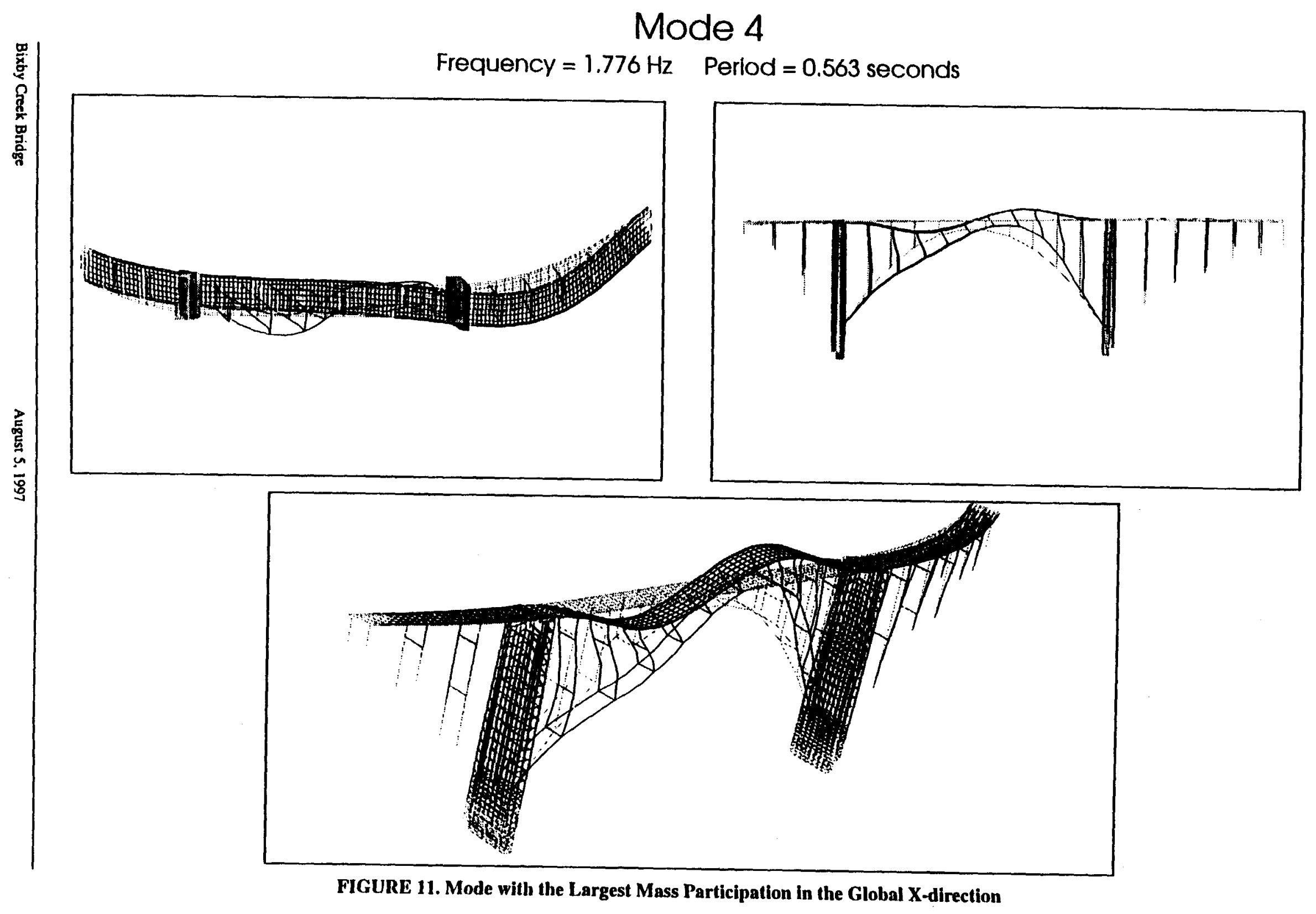




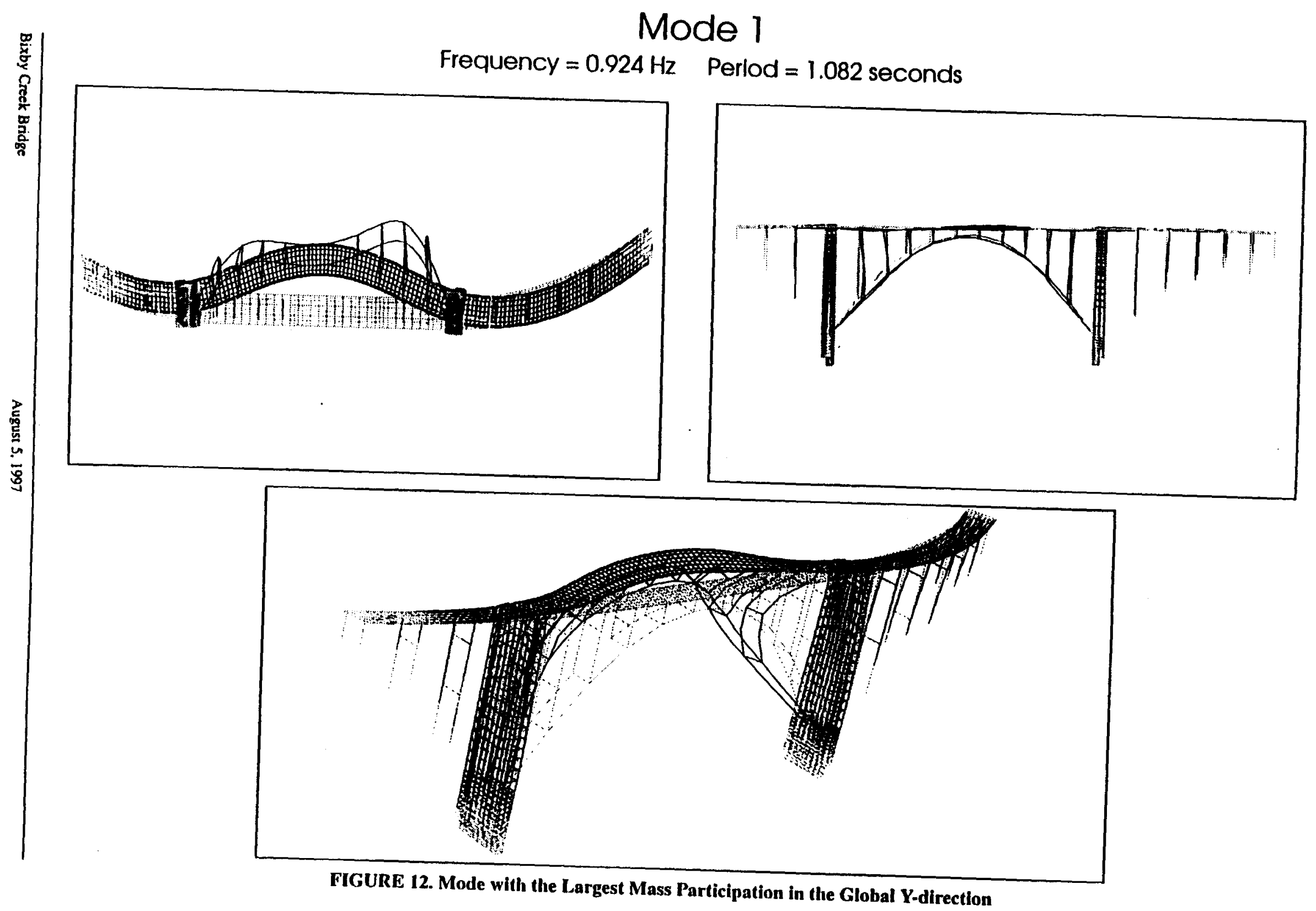




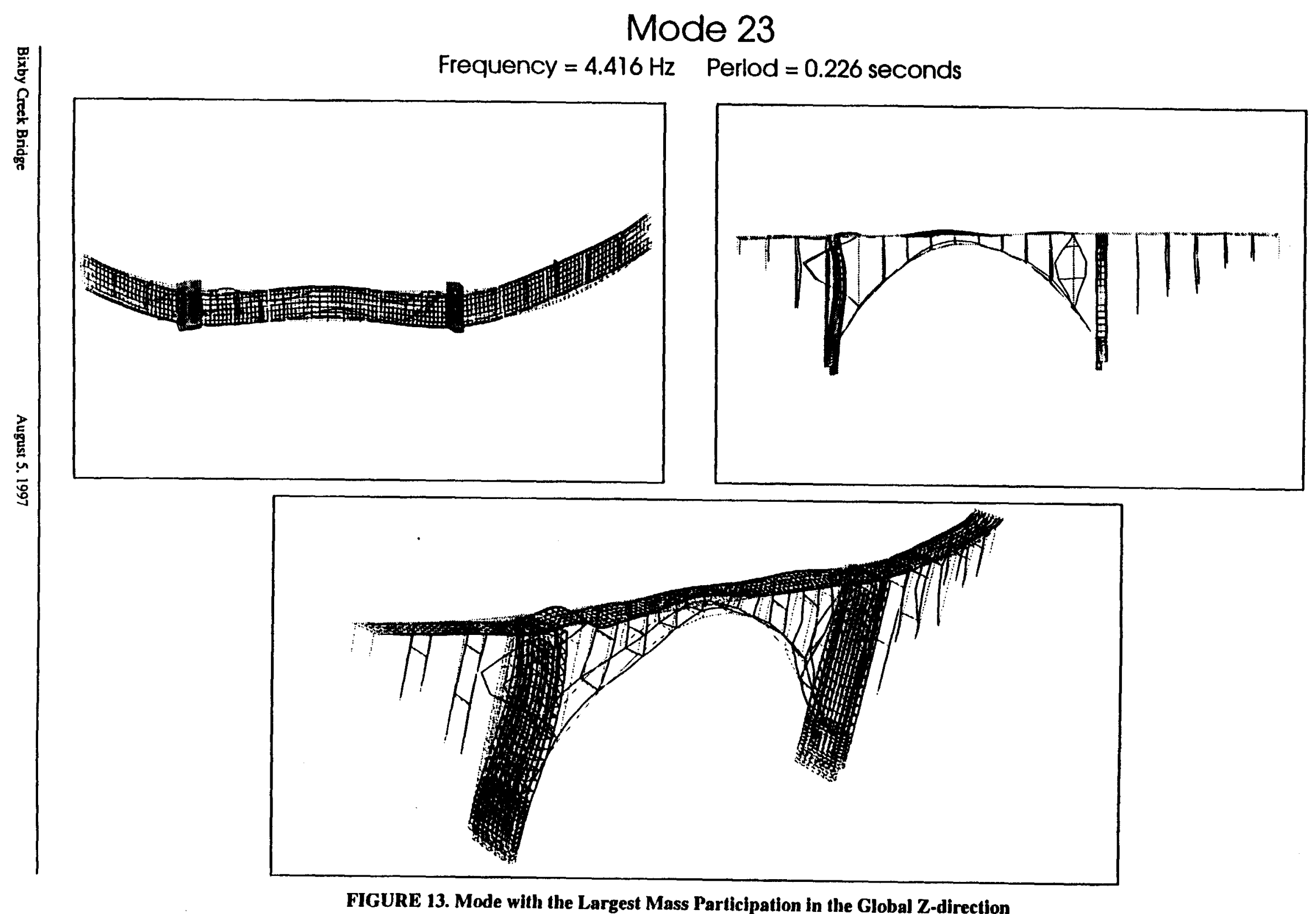


bridge will be recorded by the accelerometers and can be compared to the mode shapes predicted by the computer model. It is necessary to determine an appropriate range of input frequencies with which to vibrate the bridge. Even if this model contains some oversimplifications or modeling flaws, an input motion with a period ranging from 1.082 to 0.165 seconds is in the ball park.

In addition to being used to determined a range of frequencies for model verification tests, the natural frequencies obtained from this model will be used to determine a frequency range for recording input ground motion to be used with the empirical Green's function method. The empirical Green's function method uses ground motion response from microearthquakes $(M<3.0)$ to generate "scaled-up" versions of the microearthquake which accurately simulate site-specific, large magnitude $(M>3.0)$ earthquakes [Ref 1$]$. Similarly to the case for the model verification tests, microearthquakes with a period of around 1 second will be most important for the Bixby Creek Bridge.

Finally, the mode shapes of the structure, three of which are given above, will be used to start to formulate an idea about how the bridge will behave in an earthquake and where the sections of the bridge most likely to fail are located. It is apparent from the mode shapes that the main arch span will undergo the largest deformations when there is dynamic motion of the bridge. There is relatively very little movement in either of the approaches in the first 35 modes. Only in a few of the higher modes do the two longest approach columns begin to undergo displacement.

\section{SUMMARY}

This report documents the generation of the linear model of the Bixby Creek Bridge for the study that LLNL will conduct for Caltrans. Many of the modeling assump- 
tions presented in this report are discussed in more detail in a companion report [Ref 4]. The natural frequencies for the first 35 modes of the structure were determined and they were found to range from 1.082 second to 0.165 seconds. Pictures for critical modes of the structure are shown. 


\section{References}

1) Hutchings, L.J., Modeling Near-Source Earthquake Ground Motion With Empirical Green's Functions, Ph. D. Thesis, SUNY Binghamton, Binghamton, New York (1987).

2) State of California Department of Transportation, "Project plans for construction on State Highway," California, (1980).

3) State of California Department of Public Works Division of Highways, "Bixby Creek Bridge Plans," California, (1931).

4) Hoehler, M.S., "Bixby Creek Bridge: Notes and Simplifying Assumptions," report in preparation, Lawrence Livermore National Laboratory, Livermore, CA (1997).

5) Gerhard, M.A., "SLIC--The Interactive, Graphic Mesh generator of Finite-Element and Finite-Difference Application Programs," revised from UCRL-52823 for Version 4.0, Lawrence Livermore National Laboratory, (1991).

6) Murray, R.C. and Bumpas, S., "GEMINI--A Computer Program for Elastic Two- and Three-Dimensional Static and Seismic Structural Analysis," Lawrence Livermore NAtional Laboratory, (1993).

7) State of California Department of Transportation, "Earthquake Retrofit Project 309, Bixby Creek Bridge," California, (1996). 


\title{
Reservoir Induced Seismicity at Lake Del Valle, California
}

\author{
Jennifer Hollfelder \\ Supervisor: Lawrence Hutchings \\ Building 1405, Room 1111, L-208 \\ Lawrence Livermore National Laboratory, University of California \\ Livermore, California 94550
}

\section{Introduction}

Because a correlation between seismic activity and reservoir volume was found as early as 1945, with the impoundment of Hoover Dam, seismologists have been interested in the seismic behavior resulting from fluctuations of water volume from other reservoirs (Simpson, Leith, and Scholz, 1988). Studies were done on large as well as small reservoirs to assess their potential for reservoir induced seismicity (RIS). The best documented cases of RIS are from the dams in Egypt, India, Greece and Zambia. These dams are all very large in size which adds to our description of a possible RIS site.

However, although we know that large reservoirs are likely candidates for RIS, we are still unsure about the effects of water fluctuations from smaller size reservoirs.

This article explores the possibility of RIS at Lake Del Valle, Califormia. This particular reservoir is small but could be a site for RIS because it rests on top of several faults. The impoundment of Lake Del Valle began in January of 1969 using an earthfill dam $72 \mathrm{~m}$ high to seal the reservoir off. It has a maximum capacity of $9.5 \times 10^{7} \mathrm{~m}^{3}$ and a maximum depth of 63 $m$ making it a small reservoir in comparison to those other parts of the world. This study was done to investigate the possibility of RIS at Lake Del Valle and if RIS was found to be a problem, evaluate the potential hazards. 


\section{Background of Lake Del Valle}

Lake Del Valle is a small reservoir about 10 miles southwest of Lawrence Livermore National Laboratory (LLNL) where the study took place. There has not been any significant seismic events recorded within 1-2 km of the lakes borders, although there were several large magnitude earthquakes occurring on the Greenville fault approximately $9 \mathrm{~km}$ away. Lake Del Valle began impoundment in January of 1969 and a seismometer was installed at the reservoir in 1979 which enabled us to record magnitude 0.5 earthquakes and greater.

Figure 1 is a map that I created using Generic Mapping Tools (GMT), Datathief and Kaliedagraph. The dam is located at the northwestem corner of the lake and the Lawrence Livermore Seismic Network (LLSN) seismometer is marked by a star. The box surrounding the lake is $1.5-2.0 \mathrm{~km}$ away from the border of the lake. This box will be explained later. Events for the last 18 years were plotted as small crosses on the map.

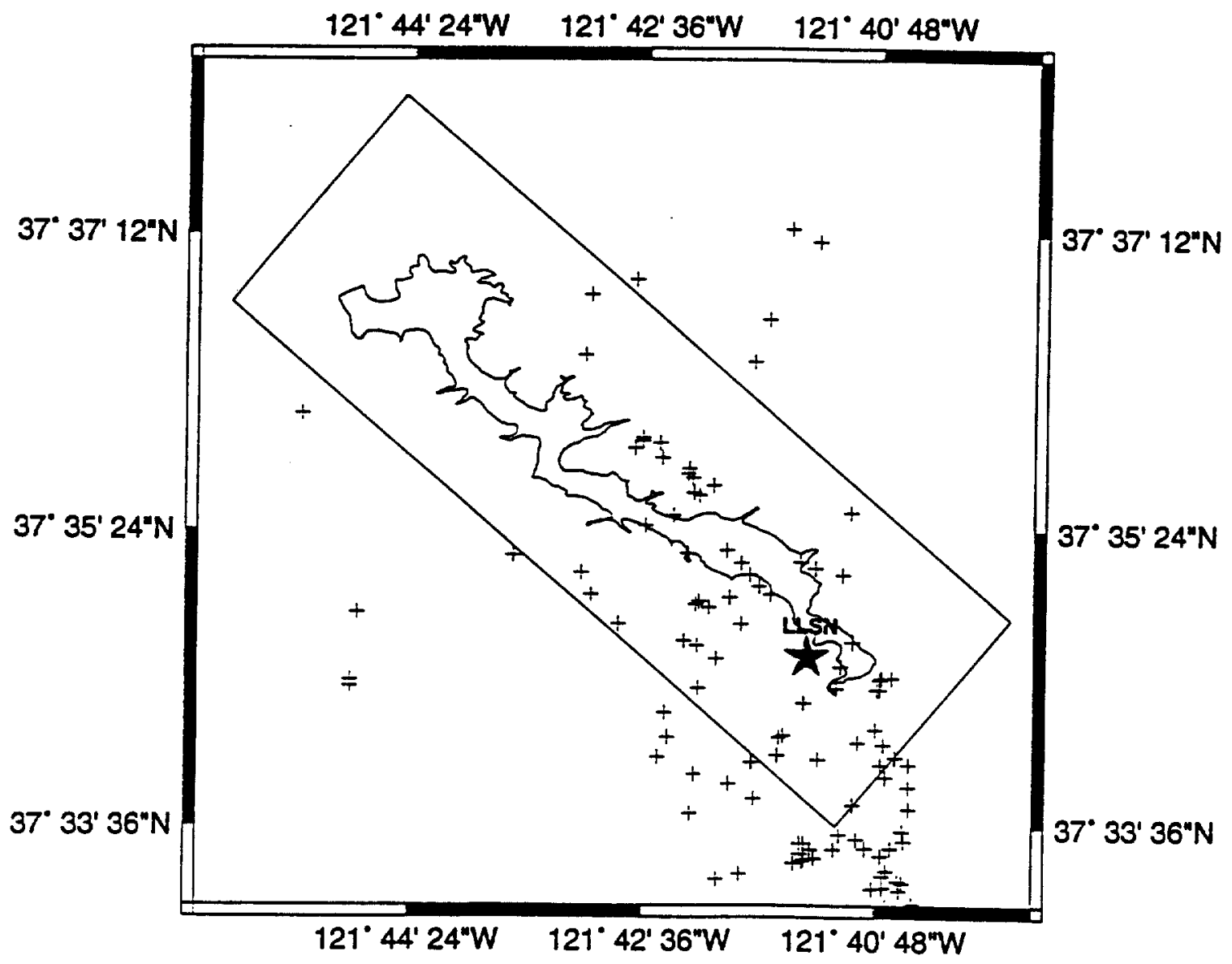

Figure 1. Lake Del Valle and every seismic event originating in the area since 1979. 


\section{A pproach}

In order to evaluate Lake Del Valle's potential to be a site for RIS, I needed to collect water volume and earthquake data for comparison. Because there was not a seismometer stationed at Lake Del Valle prior to 1979, I collected both sets of data between the years 1979 and 1996. If I had taken data since 1969 when the dam was impounded, my results would vary because only earthquakes magnitude 1.0 and greater could be detected up until 1979 . After 1979, an increase in seismicity would seem apparent because of the newly lowered detection level.

I began by creating a map of Lake Del Valle with the program GMT. After deciding on the coordinates of my map, I began searching for earthquake data on the Internet. I used the on-line UC Berkeley earthquake catalog to search for all the earthquakes originating within the coordinates of my map. I also collected monthly water volume data on-line from the Department of Water Resources (DWR). I then added a box around Lake Del Valle on my map that was $1-2 \mathrm{~km}$ away from the borders of the lake to narrow down the area for added accuracy. With a lake as small as Del Valle, the effects of the reservoir will probably not be noticeable from more than $2 \mathrm{~km}$ away.

Because I only wanted to compare events originating within the box, I wrote a program in $C$ that would pick out those events and print out how many earthquakes occurred each month. By doing this, I was easily able to compare both sets of data by graphing the date on the $\mathrm{x}$-axis and number of events and water volume on the $\mathrm{y}$-axis. I plotted all 18 years on three graphs consisting of six years each so that the data was easier to read. At this point, everything was in order to be analyzed and so the process began of making sense of it all.

\section{Finding a Correlation Between Water Volume and Seismic}

\section{Activity}

Figure 2 is a graph of the first six years of water volume and number of events vs. time data. During the first three years, there appears to be a correlation between the high water volume periods and the number of events during that month. This could be considered an indication of RIS at Lake Del Valle. However, as the years go on, it appears as if there are peaks in seismicity when the water level is at its lowest.

In figure 3 , seismic activity seems to be randomly distributed between both peaks and valleys. A clear correlation cannot be seen from this graph. In figure 4 , seismic activity experiences a serious drop off from the previous years. A high point in seismic activity corresponds with a low point in the graph. Seismic activity stays low for these final six years. 


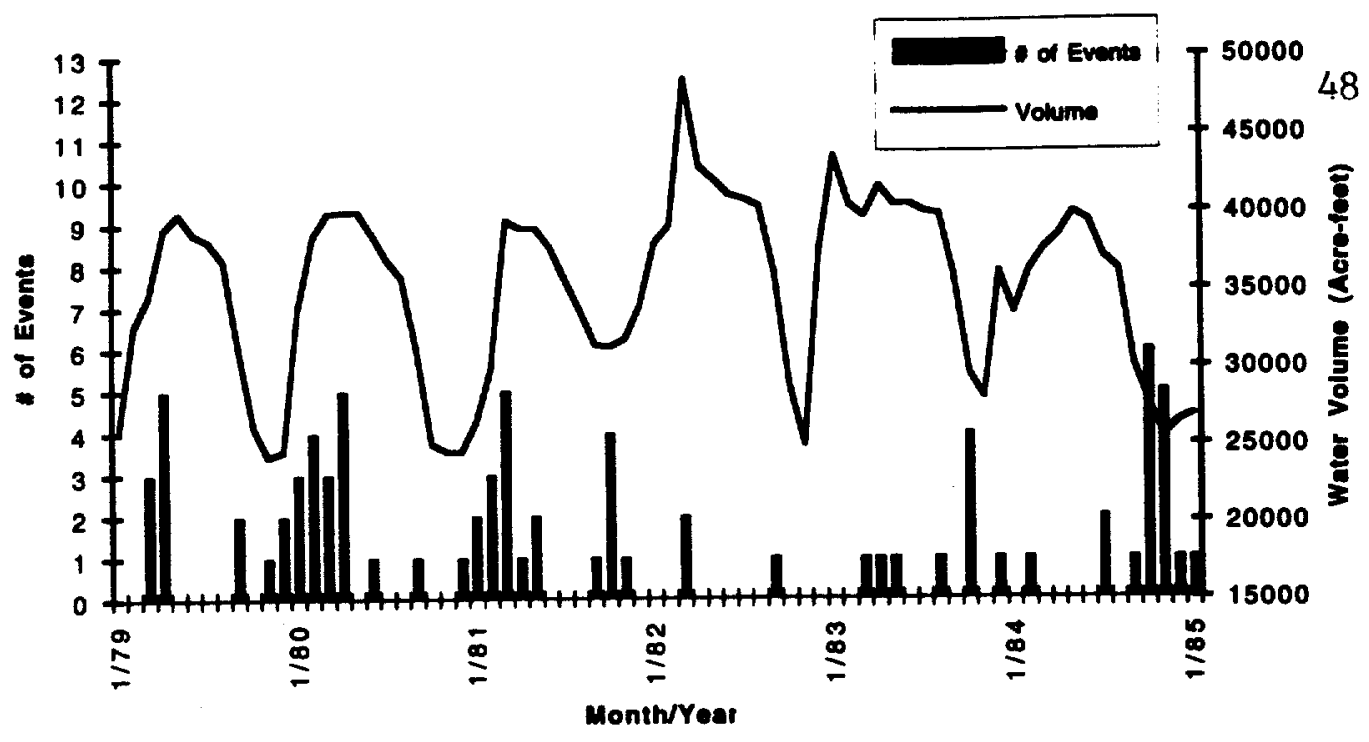

Figure 2. Graph of earthquake and water volume data between the years 1979 and 1985.

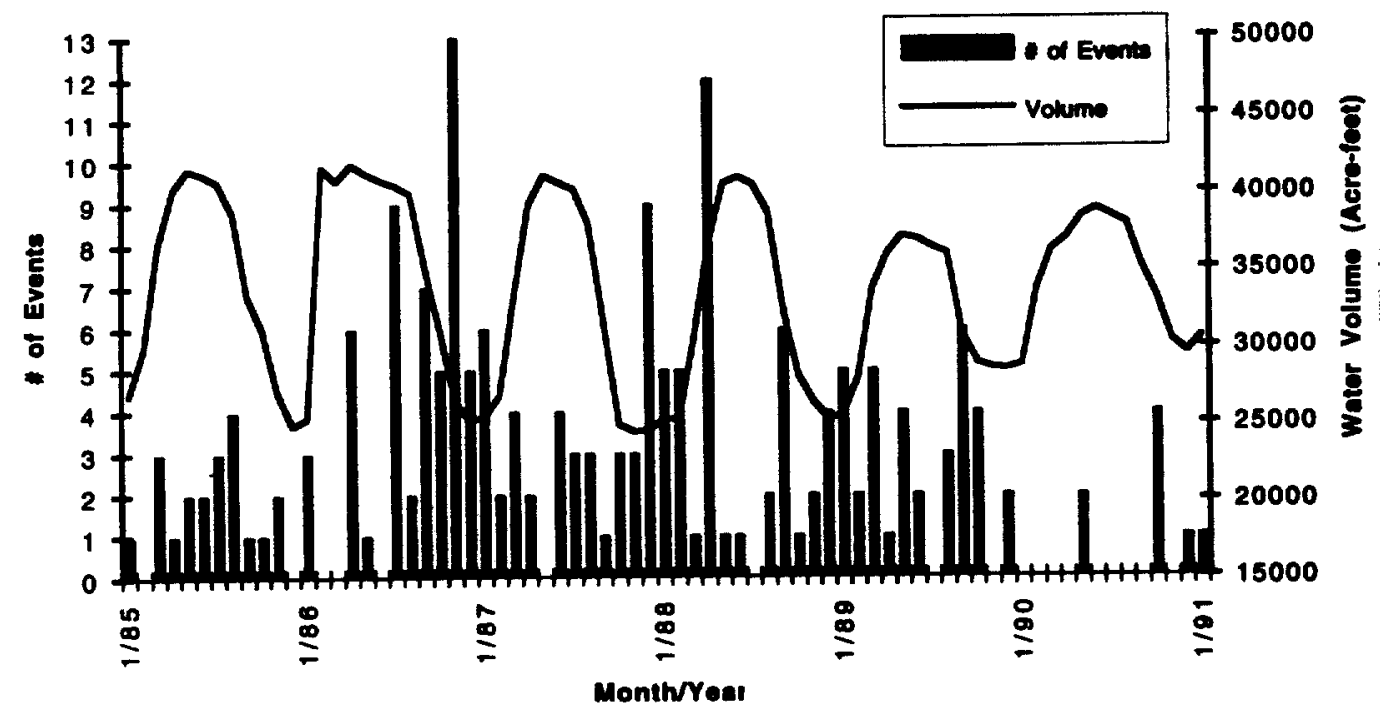

Figure 3. Graph of earthquake and water volume data between the years 1985 and 1991.

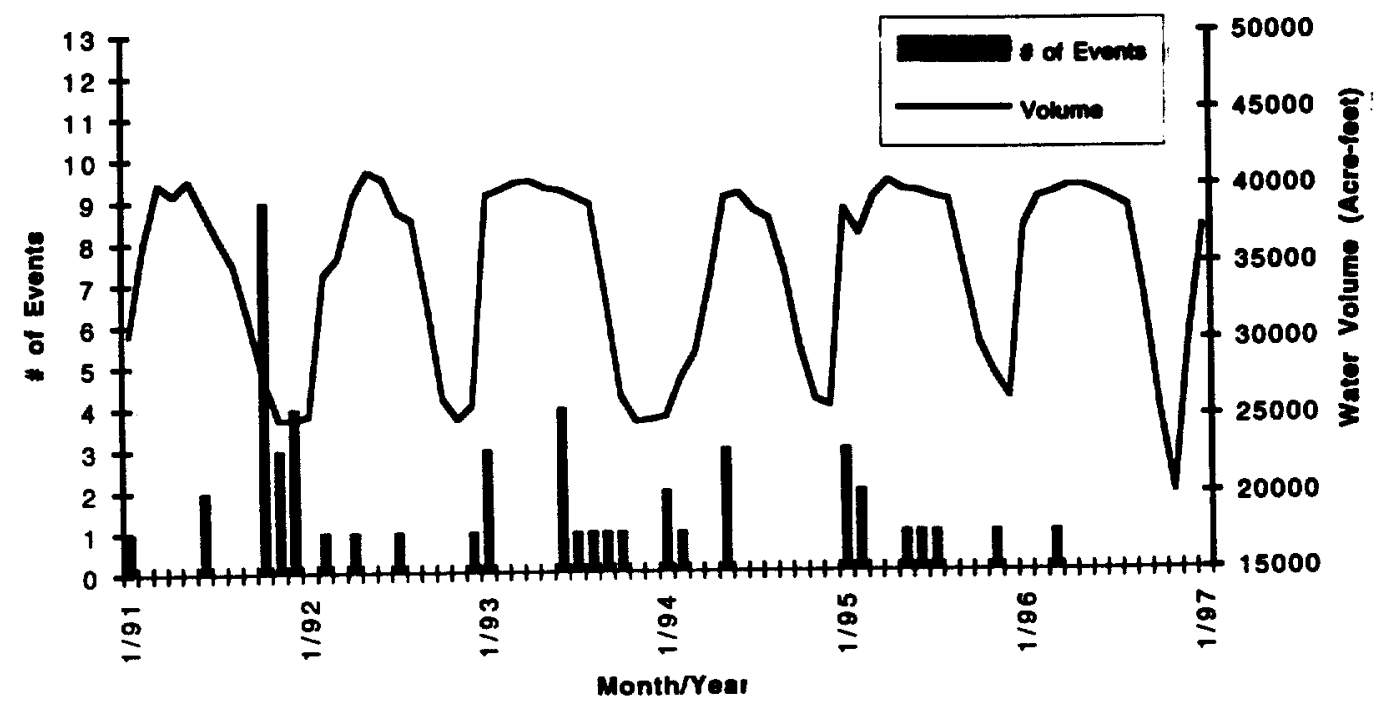

Figure 4. Graph of earthquake and water volume data between the years 1991 and 1997. 


\section{Fault Orientation}

The orientation of a fault affects its reaction to stress. If the pressure exerted on the earth is perpendicular to the fault plane, the fault is less likely to slip and will only be free to move when the pressure is alleviated. However, if the fault plane is not perpendicular to the orientation of the pressure, the fault will slip, inducing and earthquake.

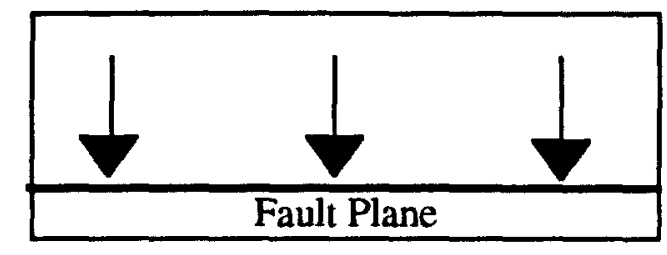

Figure 5. A fault perpendicular to the orientation of the applied pressure will stick.

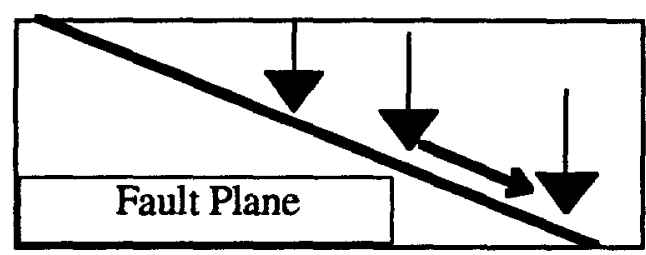

Figure 6. A fault at a diagonal to the direction of the applied pressure will slip.

The faults near Lake Del Valle could have different orientations resulting in an increase in seismicity when the reservoir experiences highs and lows in water volume.

\section{Results}

Fault orientation is the key to understanding RIS at Lake Del Valle. From looking at the graphs, I saw a correlation between water volume and number of events. When the water volume was at its highest and at its lowest, there was an increase in seismicity. This might be explained be the idea that the different orientations of a fault will cause it to react differently, therefore, inducing earthquakes during reservoir volume highs and lows. Lake Del Valle exhibits these characteristics and is a possible site for RIS. These results are not conclusive on their own though. More research and tests should be done to determine whether or not Lake Del Valle is a site for RIS. 
RIS can also be a result of the diffusion of water to hypocentral depths causing the fault to slip. This type of RIS will result in a delayed response. Both types of RIS can be in effect at any given reservoir and this would make it especially difficult to distinguish between RIS and naturally occurring events without water table and stress level data.

If I had more time to research this project, I would have looked at water table data and soil saturation levels to get an idea of how stable the ground is when the reservoir is filled. I would have also liked to have been able to create an experiment to test stress levels at the fault. These tests would have enhanced the accuracy of my paper.

Besides learning about RIS and how it is identified, I learned how to program in $\mathrm{C}$ and became very familiar with using the Internet. The most valuable thing I got out of working on this project was the ability to research a topic and be able to present it to others. Those are skills that I will be able to apply to college and work in the future.

\section{REFERENCES}

Department of Water Resources, http://cdec.water.ca.gov/misc/reservoirs.html

Northern California Earthquake Data Center, http:/quake.geo.berkeley.edu/ncedc/catalogsearch.html

Simpson, D.W., Leith, W.S., and Scholz, C.H., 1988, Two Types of Reservoir-Induced Seismicity, Bulletin of the Seismological Society of America, v. 78, p. 2025-2040.

U.S.G.S. Review of Seismic Hazard Issues Associated With the Auburn Dam Project, Sierra Nevada Foothills, California, http://water.wr.usgs.gov/auburn/auburn7.html.

Wong, Ivan G., Strandberg, James F., Assessing the Potential for Triggered Seismicity at the Los Vaqueros Reservoir, California, p. 217-231. 


\title{
QUANTUM MONTE CARLO SIMULATIONS OF THE TWO-BAND 3D HUBBARD MODEL
}

\author{
Carey Huscroft ${ }^{1}$ \\ Supervisor: Dr. Andrew K. McMahan \\ Building 121, Room 3051, L-045 \\ Lawrence Livermore National Laboratory, University of California \\ Livermore, California 94551
}

We study a remarkable structural "volume collapse" phase transition in certain rareearth materials. Combining Quantum Monte Carlo (QMC) techniques with Electronic Structure Calculations, we perform the first Q.IC study of this phenomenon using the 3$\mathrm{d}$, two-band Hubbard Model. We find the characteristic signatures of the volume collapse transition.

Certain lanthanide and actinide materials undergo "a pressure-induced crystallographic phase transition accompanied by an unusually large (up to $20 \%$ ) volume change." [1] Although this phenomenon has long been known, e.g. the $\alpha-\gamma$ transition in cerium, it remains an active area of research for which a definitive microscopic understanding has yet to be found. A leading candidate to explain this phenomenon is the Kondo volume collapse (KVC) model.[2] In this explanation, a characteristic signature of the collapse is the formation of singlet states between the material's ' $\mathrm{d}$-band' conduction electrons and its 'f-band' electrons.

The materials exhibiting this transition have such ' $\mathrm{d}$-band' and 'f-band' electrons on each atom or 'site' in the 3-d lattice comprising the material. A typical model Hamiltonian used in theoretical studies of the KVC theory is the Anderson model, which has a single impurity or ' $\mathrm{f}$-band' electron interacting with a 3-d lattice of ' $\mathrm{d}$-band' conduction electrons. While this model has the essential features required for a Kondo singlet to form between an $f$ electron and the conduction band, its simplified nature and specifically the absence of an $f$ electron on every atom mean that the effects of correlations

\footnotetext{
${ }^{1}$ University of California, Davis and Lawrence Livermore National Laboratory
} 
between 'f-band' electrons will not be present in the model and also make connection to experimental results problematic.

We overcome this problem in the Anderson model by employing a model which has a lattice of both 'f-band' and 'd-band' electrons on every site, the 3-d, two-band Hubbard model. We use Electronic Structure Calculations to calculate the relevant parameters which connect the model to real systems and then use Quantum Monte Carlo (QMC) methods to look for the characteristic signals of the KVC. QMC is a method which is exact within errorbars which can be systematically reduced by expending more and more computer resources.

In the past, such a systematic QMC study of the 3-d Hubbard Model was infeasible because of its great computational expense. However, by using the massively parallel supercomputers of the Advanced Strategic Computing Initiative along with carefully optimized simulation codes we can now perform the necessary calculations for this detailed study.

We have mapped out the phase diagram of this model via QMC for a $4 \times 4 \times 4$ system with no hybridization in the 'f-band.' We find the characteristic signatures of the KVC, a paramagnetic-anti-ferromagnetic phase transition and the formation of singlet states as hybridization between the $f$ and $d$ bands is increased.

\section{References}

[1] A.K. McMahan, Jour. Less-Comm. Metals 1491 (1988).

[2] J.W. Allen and L.Z. Liu, $\alpha-\gamma$ transition in Ce. II. A detailed analysis of the Kondo volume-collapse model, Phys. Rev. B46 5047 (1991). 


\title{
CHANGES IN PORE WATER CHEMISTRY AS A FUNCTION OF MICROBIAL GROWTH IN YUCCA MOUNTAIN TUFF
}

\author{
Anabel Miranda \\ Supervisor: Joanne Horn \\ Building 1456, Room 1035, L-206 \\ Lawrence Livermore National Laboratory \\ Livermore, California 94550
}

The US Department of Energy is currently conducting feasibility studies to asses the long term performance of a potential geological repository at Yucca Mountain, Nevada, below the ground surface in welded volcanic ash tuff. This repository must safely contain these wastes over a period of 10,000 years.

Microbial growth of both native and introduced bacteria in the potential repository site, may jeopardize the integrity geologic nuclear waste repository components and waste package materials by degrading repository materials, including cement and metal, either directly or indirectly through the alteration of pore water chemistry. Therefore, it is important to asses the impact of temperature, and nutrient conditions on microbial growth and the consequent modification of aqueous chemistry that may affect the integrity of the repository.

This study is aimed at describing and assessing the effect of bacterial activity under a range of nutrients on water chemistry and the stability of Ordinary Portland Cement (OPC) based concrete. To prepare this study we will use YM volcanic welded tuff aseptically crushed to a specified size $(2.36 \mathrm{~mm}$ $1.68 \mathrm{~mm}$ ). To determine the effects of microbes on pore water and cement, a microcosm , will be constructed and alterations in microcosm efflux chemistry as a result of bacteria growth will be determined. The impact of temperature, nutrient conditions on microbial growth and the consequent modification of aqueous chemistry will be assessed. A series of 20 separate conditions will be tested. Each experimental condition will include a sterile control whereby the ground tuff alone or with cement is exposed to identical conditions absent bacteria to account for abiotic chemical alterations. Therefore, prior to constructing microcosms, our purpose in the present work has been determine to appropriate sterilization methods. 
The sterilization technique should destroy microbial cells while, at the same time, not significantly altering the chemical and physical characteristics of the crushed rock and water system.

In order to find out the best way to sterilize tuff we used the following treatments : sodium azide, antibiotics, heat, sonication, combination of sodium azide + antibiotics, dry heat and, mercuric bromide. A negative control was used to assure no contamination from handling, and a positive control was used to assure bacteria growth when no treatment is applied. From each treatment, including the negative and the positive control, we took water samples for spectrophotometric readings $(600 \mathrm{~nm})$, and chemical analysis [Inductively couperd plasma mass spectrometry (ICPMS), ion chromatography(IC), total organic carbon (TOC)] in order to determine the presence of bacteria, if any, after each treatment and changes in rock chemistry respectively. The tuff from each treatment was washed with deionized water and again, samples for absorbency readings and chemical analysis were taken. After washing, the tuff was combined with $10 \mathrm{ml}$ of bacterial M9 growth media (M9). Three days later, samples from each culture were inoculated onto M9 plates. Also, after another three days of incubation on $\mathrm{M} 9$ plates, the plated samples were re-inoculated to another set of $\mathrm{M} 9$ plates to differentiate between light lawn ${ }^{1}$ and very fine rock powder produced by crushing.

The microbial studies show that some methods of sterilization (sodium azide, antibiotics, antibiotics + azide) will achieve the desired results (i.e. no growth of bacteria from treated tuff). However, inorganic analyses of these treatments compare to the positive and negative controls suggest that the chemical signature of these sterilization methods will overlap the chemical species of interest, which is unacceptable. Mercuric Bromide, dry heat (using an oven), and sonication do not achieve complete sterilization. Apparently, injured microbial cells or spores, treated using the methods, are able to escape killing and subsequently form colonies. The wet heat treatment (using autoclave) results are not complete. However, it is expected that the heat will alter some mineral constituents of the rock to make them more soluble. 
Clearly, even if a particular sterilization method is successful in killing all microorganisms, the chosen method will depend on what result is required since all sterilization methods produce alterations in the chemistry of the medium (in this case, YM tuff), the method of choice will depend on what specific chemical species one wishes to analyze.

Joanne Horn, Annemarie Meike (1995) Microbial Activity at Yucca Mountain. Yucca Mountain Site Characterization Project. Report UCRL-ID-122256.

Angel Rivera, (1996), Testing Candidate Alloys for Resistance to Microbial Induced Corrosion. Lawrence Livermore National Laboratory Paper, Science and Engineering Semester Program. 


\title{
NO SIGNIFICANT EVIDENCE FOUND FOR A DNA FINGERPRINT FROM DENSELY IONIZING RADIATION BASED ON F RATIOS CALCULATED FROM STABLE CHROMOSOME ABERRATIONS
}

\author{
S. W. Oram, F. S. Hill, A. M. Chen, and J. N. Lucas \\ Supervisor: Joe Lucas \\ B361, R1950, L-452 \\ Lawrence Livermore National Laboratory \\ Livermore, CA 94550
}

\section{INTRODUCTION}

The purpose of this study is to determine if there is a DNA "fingerprint" for densely ionizing radiation by measuring the ratio of interchromosomal to intrachromosomal exchange-type aberrations (F ratio). Chromosome aberrations produced by ionizing radiation are assumed to develop from DNA double-strand breaks (DSBs) which interact pairwise (Sachs et al . 1993). Dicentrics and translocations are interchromosomal aberrations, caused by breaks in different chromosomes (Figure 1); while centric rings and pericentric inversions are intrachromosomal aberrations, caused by multiple breaks in the same chromosome (Figure 2). Centric rings and dicentrics are unstable aberrations, which are almost always chronologically fatal. Translocations and pericentric inversions, on the other hand, are stable aberrations that persist in lymphocytes for long periods of time (Lucas et al. 1992, Lucas et al. 1996). For this study we measured translocations and pericentric inversions because: 1) They are stable over time and 2) The F ratio comparing high- and low- LET radiation, using translocations and pericentric inversions, has not been established. In a recent commentary, Brenner suggested that densely ionizing radiation leaves a distinct chromosomal marker that may be detected and measured long after radiation exposure 
because it produces an anomalously low $\mathrm{F}$ ratio in comparison with $\mathrm{X}$ rays or chemical carcinogens (Brenner 1994). If DSBs are produced randomly in a human cell, and assuming all the DSBs are equally likely to interact with each other, the $\mathrm{F}$ ratio would be approximately 82, based on human chromosome arm lengths (Hlatky et al. 1992). However it has been demonstrated that the actual F ratio for low-LET radiaition is believed to be around10-15 (Schmid and Bauchinger 1996; Brenner 1996). This can be explained by the fact that individual chromosomes are localized within subdomains that are smaller than the cell nucleus, which will produce a bias toward intrachromosomal aberrations (centric rings and pericentric inversions) relative to interchromosomal aberrations (dicentric and translocations). However, densely ionizing radiation (high-LET radiation) produces energy depositions that are much closer together than those produced by gamma or X rays (low-LET radiation). Hence, it may be more likely for high-LET radiation to cause multiple breaks in the same chromosome than it is for low-LET radiation. Leading to an even further reduced $\mathrm{F}$ ratio for high-LET radiation and therefore facilitating a "fingerprint" for densely ionizing radiation. With this idea in mind Brenner suggested that measurements of peripheral lymphocytes of survivors at Hiroshima provides "direct biological evidence that the neutron component at Hiroshima not only is significant, but dominates the total effective dose at the ground distances of most relevance for risk estimation" (Brenner 1996).

Later, Schmid and Bauchinger rebuked these ideas by examining the ratio of dicentrics to centric rings for different levels of $\mathrm{X}$ rays, gamma rays, neutrons and alpha particles. They concluded that $F$ ratios could not provide reliable evidence for a DNA "fingerprint" for densely ionizing radiation (Schmid and Bauchinger 1996). Brenner however believes that Schmid and Bauchinger did not examine a low enough dose of highLET radiation. He suggests that as the dose of high-LET radiation goes up, so does the number of high-LET tracks within the nucleus. Causing the spatial distribution of chromosome breaks to look more and more like low-LET radiation. Therefore for a DNA 
"fingerprint" to be present, one must examine low dose high-LET radiation, where few nuclei would be exposed to multiple high-LET tracks (Brenner and Sachs 1996).

In this study we consider Brenner and Sach's suggestion and examine the $\mathrm{F}$ ratios (ratio of translocations to pericentric inversions) for low-LET, low dose high-LET and high dose high-LET radiation.

\section{METHODS AND MATERIALS}

FISH ANALYSIS: A rapid and accurate method for measuring pericentric inversions using FISH is described (Lucas et al. 1996). The method to measure pericentric inversions employs fluorescent probes generated by degenerate oligonucleotide-primedpolymerase chain reaction (DOP-PCR). We used DNA probes specific for the heterochromatin at $1 \mathrm{q} 12$ (pUC 1.77) labeled in green, a p arm telomere region (1p36.3) on chromosome number 1 also labeled in green, in combination with a pan-centromere probe labeled in red (Figure 3). The pan-centromere probe was amplified and directly labeled with Tetramethylrhodamine-6-dUTP (Boehringer Mannheim) by DOP-PCR. The pUC 1.77 DNA for the heterochromatin and the $\mathrm{p}$ arm telomere DNA were also amplified by DOP-PCR. The telomere and pUC 1.77 probes were then labeled with FluoroGreen (Amersham) in a second round of amplification. Details of the hybridization procedures are as described in Pinkel et al. (1986). Briefly, the target metaphase spreads were denatured in $70 \%$ formamide $/ 2 \mathrm{XSSC}$ at $72^{\circ} \mathrm{C}$, dehydrated and air-dried. The directly labeled probes in hybridization buffer ( $50 \%$ formamide/2XSSC, $10 \%$ dextran sulfate) were thermally denatured at $72^{\circ} \mathrm{C}$ and added to the slides. The cover slips were sealed, and the slides were incubated overnight at room temperature. After washing, the slides were counterstained with 2,6-diamidino-2-phenylindole (DAPI). 
CELLCULTURE: Procedures are as described in Lucas et al. (1989). Heparinized whole blood from a healthy male donor was irradiated at ambient room temperature with different levels of high- and low- LET radiation. Absorbed doses to the blood in Gy were obtained from procedure described in Lucas et al. (1989). Lymphocytes were seperated from whole blood of a healthy male donor by centrifugation through a continuous density gradient formed by mixing whole blood with a commercially availabe seperation medium (Sepracell-MN, Sepratech Co., Oklahoma City, OK). The lymphocytes were stimulated to proliferate with phytohaemagglutinin $(0.15 \mathrm{mg} / \mathrm{ml})$. The cells were maintained in suspension cultures in upright T-75 flasks in $50 \mathrm{ml}$ RPMI 1640 medium containing $20 \%$ fetal bovine serum, $1 \%$ pen/strep/glu and 1.5\% PHA. After 48 hours, colcemid was added to the cultures for 4 hours, and metaphase spreads were prepared by the method of Evans et al. (1971).

SCORING: For pericentric inversion identification a metaphase spread was scored as normal if green fluorescent domains positioned on opposite sides of a red fluorescent centromere were present on each of the two number 1 chromosomes. Two kinds of chromosome rearrangements involving chromosome 1p were scored: (1) Pericentric inversions with one breakpoint in $1 \mathrm{p}$ and the other in $1 \mathrm{q}$; these produced a derivative chromosome with a visible change in the color pattern (Figure 3). (2) Centric rings with one breahpoint in $1 \mathrm{p}$ and the other in 1q; these produced centric rings with the ring carrying the paracentromeric locus of chromosome 1 and an accentric fragment containing the telomeric locus. All metaphase spreads containing structural aberrations involving $1 \mathrm{p}$ were photographed and the scoring was reviewed in conference.

Identification and scoring of translocations using FISH employed the methods of Lucas et al. (1992). Only reciprical translocations were used in determing the translocation frequency. 


\section{GENOMIC TRANSLOCATION AND PERICENTRIC INVERSION}

CALCULATIONS: Identification and scoring of chromosome exchange aberrations using FISH employed the methods of Lucas et al. (1992). The scoring criteria are equivalent to those described by Simpson and Savage (1995) as apparently simple exchange-type painting pattern. Only apparently simple translocations (ASTs) were used to calculate F-ratios (Lucas et al. 1996). The translocation frequency measured by FISH was scaled to full genome equivalents using the Lucas et al. formula (Lucas et al. 1992). In brief, the formula relates the translocation frequency measured by FISH, $\mathrm{F}_{\mathrm{p}}$, to the genomic translocation frequencies, FG, through the fraction of the genome covered by the probes, $f_{p}$, as follows:

$$
F_{G}=F_{p} /\left(2.05 f_{p}\left(1-f_{p}\right)\right)
$$

where the genomic conversion factor for chromosome 1 is 0.156 .

The FISH measured pericentric inversions, $\mathrm{p}_{\mathrm{i}}$, involving the ith chromosome is proportional to the product of the long $\left(\mathrm{L}_{\mathrm{i}}\right)$ and short $\left(\mathrm{S}_{\mathrm{i}}\right)$ arms of the ith chromosome. The total genomic number of pericentric inversion, $\mathrm{P}$, is obtained similarly (Hlatky $e t$ al. 1992) from the ratio of the product of the long and short arms of the ith chromosome to the sum of products of the long and short arms for the total genome to the product of the long and short arms of the ith chromosome, times the FISH measured pericentric inversions, $\mathrm{p}_{\mathrm{i}}$, involving the ith chromosome: where $\mathrm{L}_{\mathrm{i}}$ and $\mathrm{S}_{\mathrm{i}}$ are the long and short arms of the ith chromosome.

$$
\mathrm{P}=\mathrm{p}_{i} \sum_{j=1}^{46}\left(\mathrm{~L}_{j} \mathrm{~S}_{j}\right) / \mathrm{L}_{i} \mathrm{~S}_{i}
$$




\section{RESULTS}

Cultures of human lymphocytes, which were stimulated to grow as described previously (Hill et al. 1994), produced excellent metaphase spreads with 100 to 500 metaphase spreads per slide. The use of the heterochromatin (pUC 1.77) probe labeled in green, the pan-centromere probe labeled in red and the $1 \mathrm{p}$ telomere $(1 \mathrm{p} 36.3)$ probe labeled in green allowed for easy and quick identification of pericentric inversions. A normal chromosome 1 consisted of a green-red-green pattern, while a inverted chromosome 1 contained a green-green-red pattern. The use of human whole chromosome paint probes allowed for easy identification of reciprical translocations by using FISH methods employed by Lucas et. al (1992).

Table 1 lists the dose, number of translocations and pericentric inversions, translocation frequencies, pericentric inversion frequencies and $\mathrm{F}$ ratios for both high- and low-LET radiation. For the 2.89 Gy Co-60 gamma irradiation (low-LET radiation), 1966 metaphase lymphocytes were scored. There were 24 pericentric inversions and 140 reciprocal translocations. Resulting in a translocation frequency of 0.53 and a pericentric inversion frequency of 0.083 . Indicating a $F$ ratio (translocation frequency/ pericentric inversion frequency) of 6.39 .

The high-LET radiation Iron (Fe-56) study consisted of two different doses $(0.25$ Gy and $1.25 \mathrm{~Gy}$ ). Data for the $0.25 \mathrm{~Gy}$ dose consisted of 8682 metaphase lymphocytes, with 16 pericentric inversions and 38 translocations. Resulting in a translocation frequency of 0.065 and a pericentric inversion frequency of 0.015 . Thus giving a $F$ ratio of 4.33. The other group consisting of a dose of $1.25 \mathrm{~Gy}$ had 4922 metaphase lymphocytes with 21 pericentric inversions and 32 translocations. Resulting in a translocation frequency of 0.160 and a pericentric inversion frequency of 0.036 . Indicating a $\mathrm{F}$ ratio of 4.44 . 
The high-LET radiation Carbon (C-14) study contained 1070 metaphase lymphocytes, resulting in 16 pericentric inversions and 31 translocations. Thus giving a translocation frequency of 0.820 and a pericentric inversion frequency of 0.123 . Resulting in a F ratio of 6.67 .

Table 2 shows the results of statistical analysis of the $\mathrm{F}$ ratios produced from this study. In comparing the $\mathrm{F}$ ratios for low-LET ( $2.89 \mathrm{~Gy}$ Co-60) radiation to the different levels of high-LET ( 0.25 and $1.25 \mathrm{~Gy} \mathrm{Fe-56}$ and $3.00 \mathrm{~Gy} \mathrm{C}-14)$ radiation, the $P$ values are $0.968,0.958$ and 0.993 respectively. Indicating that there is no significant difference between the $\mathrm{F}$ ratios for low- and high-LET radiation.

TABLE 1: Data for Inversion Paper.

Cells

High-LET Radiation

Iron (Fe-56)

Lymphocytes

Dose Cells Trans Trans. Freq Inv Inv. Freq F-ratio

$\begin{array}{lllllll}0.25 & 8682 & 38 & 0.065 & 16 & 0.015 & 4.33 \\ 1.25 & 4922 & 32 & 0.160 & 21 & 0.036 & 4.44\end{array}$

High-LETRadiation

Carbon (C-12)

Lymphocytes

3.00

1070

$34 \quad 0.820$

16

0.123

6.67

Low-LET Radiation

Gamma Rays (Co-60)

Lymphocytes

2.89

1996

140

0.530

$24 \quad 0.083$

6.39 
Table 2: P-Values for inversion paper

$\begin{array}{llll}\text { Fe-56 } & \text { Fe-56 } & \text { C-12 } & \text { Co-60 } \\ 0.25 \text { Gy } & 1.25 \text { Gy } & 3.00 \text { Gy } & 2.89 \text { Gy }\end{array}$

\section{$\underline{\mathrm{Fe}-56}$}

$0.25 \mathrm{~Gy}$

$\ldots \quad 0.998$

0.832

0.968

$1.25 \mathrm{~Gy}$

0.998

$\cdots$

0.951

0.958

C-12

$3.00 \mathrm{~Gy}$

0.832

0.951

$\cdots$

0.993

Co-60

$2.89 \mathrm{~Gy}$

0.968

0.958

0.993

\section{DISCUSSION}

Using three common DNA probes: a 1p telomere probe(green), a heterochromatin(pUC 1.77) probe(green) and a centromeric probe(red) we measured the pericentric inversions for low- and high-LET radiation. We then compared the translocation frequency, that was measured by using whole chromosome paint probes, to the pericentric inversion frequency. This allowed us to compare the interchromosomal exchange-type aberrations to the intrachromosomal exchange-type aberrations (F ratio) for low- and high- LET radiation to determine if there was a "fingerprint" for high-LET radiation. There has been conflicting opinions about this subject in the past. Brenner(1994 and 1996) has predicted that the $F$ ratio for high-LET radiation would be lower than the $\mathrm{F}$ ratio for low-LET radiation. Schmid and Bauchinger (1996) disagree with this idea and have published data denying this claim based on data featuring the ratio 
of dicentrics to centric rings. However, they did not examine low dose high-LET radiation, where Brenner believes the "fingerprint" exists.

In this study we examine the $\mathrm{F}$ ratios for low dose high-LET radiation, high-dose high-LET radiation and high dose low-LET radiation. From our data there is no "fingerprint" for high-LET radiation by comparing F ratios. Low-LET (Co-60 2.89 Gy) radiaiton produced a F ratio of 6.39; while high-LET radiation, $0.25 \mathrm{~Gy} F E-56,1.25 \mathrm{~Gy}$ Fe-56 and 3.00 Gy C-12, produced F ratios of 4.33, 4.44 and 6.67 respectively. Statistical analysis of the F ratios for low-LET radiation(Co-60 2.89 Gy) and the three doses of high-LET radiation(Fe-56 0.25 Gy, Fe-56 $1.25 \mathrm{~Gy}$ and C-12 3.00 Gy) produced $P$ values of $0.968,0.958$ and 0.993 respectively. Demonstrating that the $F$ ratio for lowLET is not significantly different from the values for high-LET. The F ratios for highLET (Fe-56 0.25 and 1.25 Gy) are slightly lower than the F ratios for low-LET (Co-60). This slight decrease can be explained by a large number of non-reciprical translocations for the Fe-56 groups. Which were not used to calculate translocation frequencies, only reciprical translocations were used to determine the translocation frequencies.

In summary, we conducted research to determine if a "fingerprint" for densely ionizing radiation existed by comparing $F$ ratios for high and low dose high-LET radiation with low-LET radiation. We examined a 3 doses of high-LET radiation: 0.25 Gy (Fe-56), where we believe few nuclei would be exposed to multiple high-LET tracks, 1.25 Gy (Fe56) and $3.00 \mathrm{~Gy}(\mathrm{C}-12)$. We found no "fingerprint" at any level. During the course of the study we also realized that scoring requirements for low dose radiation (high- or lowLET) are massive. Leading us to believe that at any dose lower then $0.25 \mathrm{~Gy}$ the scoring requirements would be to immense for a "fingerprint" to be useful. We do not believe that a "fingerprint" exists at lower doses than $0.25 \mathrm{~Gy}$, but if it did the large amounts of scoring to identify the "fingerprint" would render the process useless for laboratory biodosimetry. 


\title{
The Yucca Mountain Project Small Block Tests or \\ Nuclear Waste: It's not just for breakfast anymore!
}

\author{
Adam L. Rosenberg \\ Supervisor: Dr. Stephen Blair \\ Building 1402, Rm 1016, L-201 \\ Lawrence Livermore National Laboratory \\ Livermore, CA 94550
}

\section{Introduction}

I have no statistical evidence to back this up, but I believe experts and laymen agree that radioactive waste is not something they want popping up in their drinking water. If we could wish it all away, we probably would. Unfortunately, that's simply not possible, and over the last fifty years, over 380,000 cubic meters of the stuff has accumulated in the US alone (Whipple, 1996). And with the number of operating domestic nuclear reactors up to 109 today, that amount is only going to increase at an even greater rate in the years to come (United States Energy INFOcard, 1995). We have to find a safe way to get rid of it on a reasonable timescale, and that's where the Yucca Mountain Project comes in.

At Yucca Mountain, in the middle of the desert of southern Nevada, the Department of Energy is considering a plan to bury thousands of tons of radioactive waste several hundred meters below the earth's surface. Yucca Mountain's dry climate, below-average groundwater table, and isolation from civilization make it a promising location for a nuclear waste repository, but our primary concern is that over the next few thousand years these conditions may change. If water reaches the radioactive containers, it may cause them to corrode, and then could act as a carrier for the radionuclides within. We want to find out if this water could ever reach civilization, and to do that the Yucca Mountain Project has 
several tests on various scales and under various conditions to study groundwater flow inside the rock.

Thermal, mechanical, and hydrological processes are all coupled together and play a role in this investigation. Temperatures over $200^{\circ} \mathrm{C}$ will be found in the rock due to heat generated by the containers. Near the containers this should cause the water to vaporize. However, the thermal expansion in the rock may cause the fractures allowing water flow to open, and high temperatures may also make the rock weaker, allowing the water to erode it more easily. Stress changes are caused by excavation in the rock for container placement. Higher stresses may close the fractures, inhibiting water flow. As evidenced by the proliferation of the word "may", though, further investigation is clearly required.

This paper will mainly focus on the second of the small block series of experiments. The first of the series provided new deformation and elastic wave velocity data which permit approximation of total rock mass properties and behavior for Yucca Mountain rock that contains fractures and vugs. (Vugs are similar to large bumpy holes inside and on the walls of the block.) It also provided guidance for input values used in equivalent continuum models of a repository (Blair and Costantino, 1997). The current block, also cut from the Yucca Mountain site, is about $26.2 \mathrm{~cm} \mathrm{x} 24.5 \mathrm{~cm} \mathrm{x} 49 \mathrm{~cm}$ and has a large through-going artificial fracture in the middle of the z-direction, parallel to the ground. We want to test water flow through this fracture under various stresses and temperatures. For guidance in our experimental procedure, we have a theory.

\section{Theory}

We are setting up this experiment with a particular theory in mind. The idea is that the mass flow rate of water out of the block is proportional to the driving pressure of water into the block multiplied by the width of the fracture aperture cubed, or:

$$
\mathrm{dm} / \mathrm{dt}=\mathbf{k} \mathbf{P}_{\mathrm{in}} \mathbf{h}^{3}
$$


Figure 1.

\section{Theoretical Flow Rate vs. Fracture Width Plot}

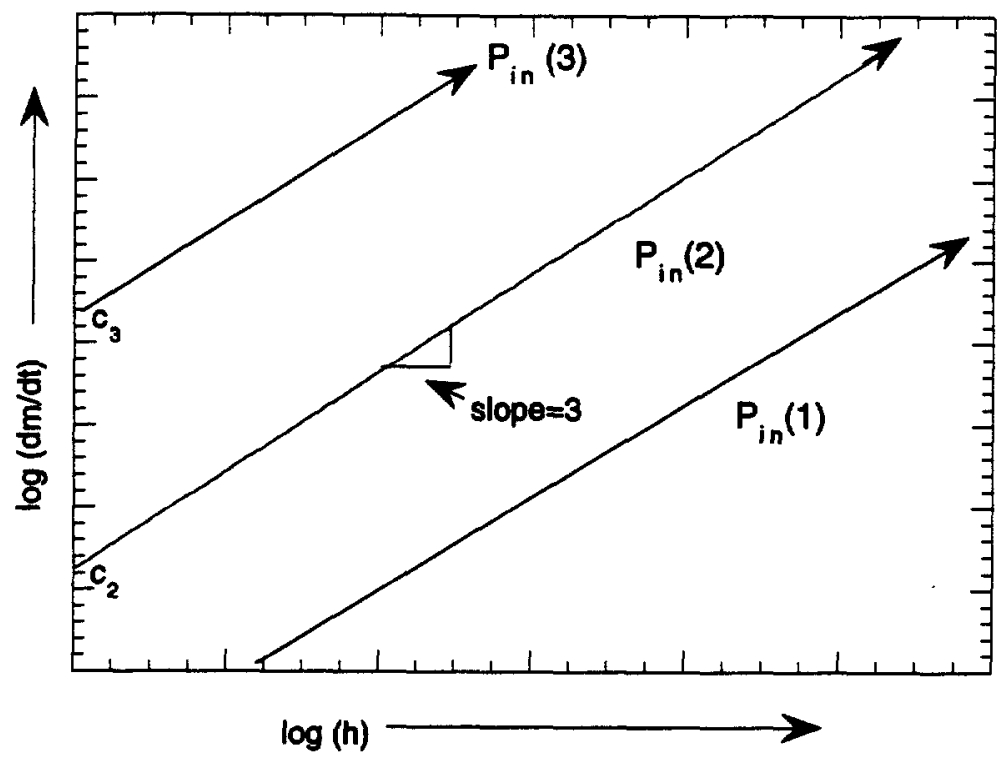

where $\mathrm{k}$ is a currently unknown multiplication constant depending on the geometry of the block and inversely proportional to the viscosity of the fluid (Anderson and Woessner, 1992). Rewriting this equation, we get:

$$
\begin{gathered}
\log (\mathrm{dm} / \mathrm{dt})=\log \left(k \mathrm{P}_{\mathrm{in}}\right)+3 \log (\mathrm{h}), \text { or: } \\
\log (\mathrm{dm} / \mathrm{dt})=\mathrm{c}_{1}+3 \log (\mathrm{h})
\end{gathered}
$$

Graphs of this equation should look something like Figure 1, with each line representing a different $P_{\text {in }}$. To explain the relation of the above equation to thermal and mechanical processes, aperture width is hypothesized to be proportional to vertical stress (thus stress changes are applied perpendicular to the fracture plane). Also, temperature may change $c_{1}$ by changing the fluid viscosity, and it changes the aperture width due to thermal expansion by replacing $h$ to first order with $h(1+\alpha \Delta t)$, where $\alpha$ is the coefficient of thermal expansion of the block, and $\Delta t$ is its change in temperature. (Temperatures over the boiling point of water will change the above equation significantly, but that event will not be explored in this report.) So most of the major processes investigated can be seen in this one graph. Of 
course, this is only a theory, with simplistic boundary conditions of completely parallel rock faces around a fracture, and we are currently testing the theory's validity.

\section{Methodology}

Many different instruments and systems are used to carry out and analyze the small block test. The block sits in a large loading frame which can apply up to 300 tons of force on the block over the fracture plane if we so desire. A 10,000 psi pump provides that pressure to the loading frame.

16 LVDTs (Linear Voltage Displacement Transducers) are placed all around the block. (See Figure 2 for layout.) All on the north and south face of the block are vertical, most measuring displacement across the fracture plane. Those on the east face surround a large vug, while those on the west are used as a general horizontal and vertical diagnostic. Extensions of .125 " diameter quartz rods were used when the LVDT device was not long enough to span the desired distance.

Twelve 0.062" diameter Type J thermocouples were mounted in 0.125 " diameter holes on a grid, as shown in Figure 3. The thermocouple junctions lie in a vertical plane parallel to the south face and at the center of the sample. Two more thermocouples lie above the block, and two below (Blair and Costantino, 1997).

Information from the LVDTs, thermocouples, and loading cell sensor is sent through a data acquisition system, consisting of two National Instruments (NI) Mdl SCXI 1303 Low Thermal terminal blocks connected to two NI Mdl SCXI 1100 multiplexors, which send information to a NI NB MIO-16L, 12-bit A/D card in a MacIntosh MacIlfx computer that reads, displays, and stores the data using NI LabView software (Blair and Costantino, 1997).

The fluid flow system, shown in Figure 4, is comprised of a pressurized reservoir, a point source at the center of the fracture, and a collection manifold along the fracture perimeter. Fluid pressure of up to $80 \mathrm{psi}$ is controlled to within .1 psi using a regulator and 


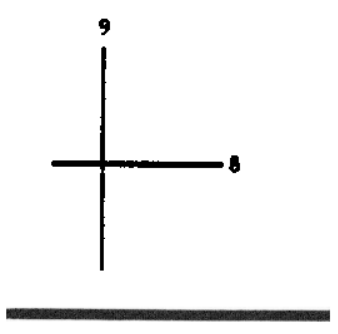

Enat Face

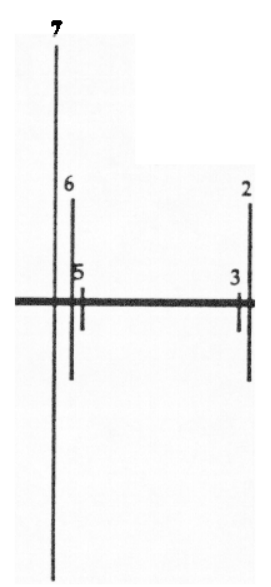

North Face
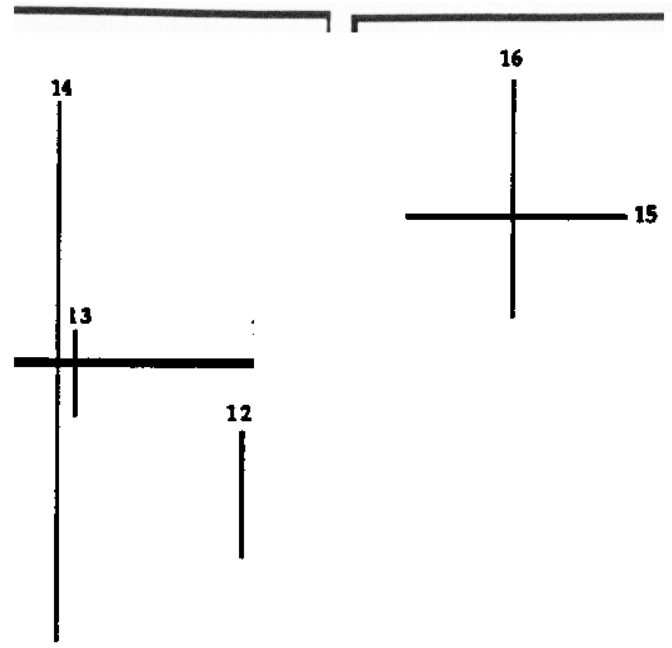

Figure 2. SB3 LVDT Layout

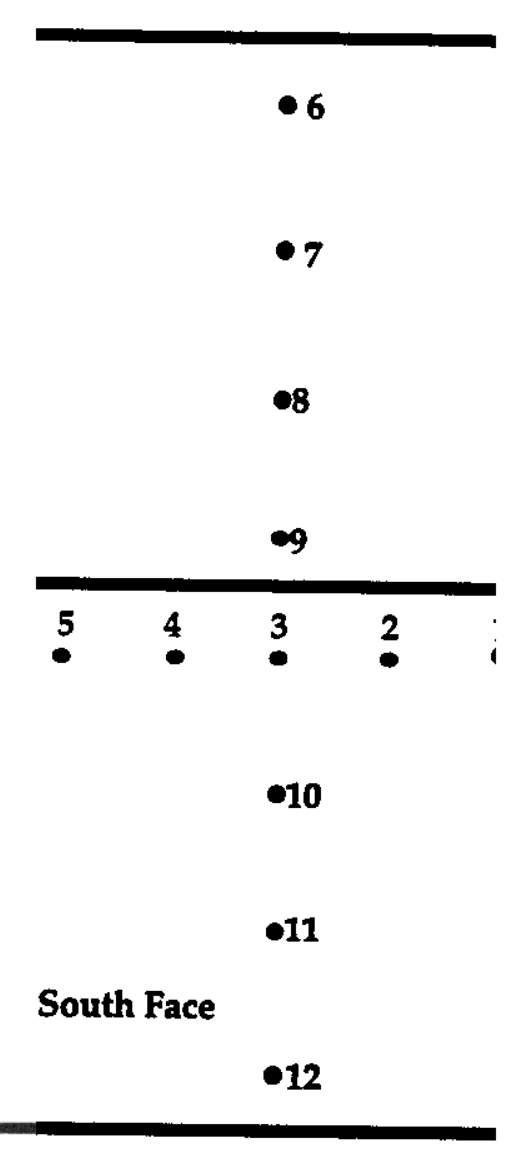

Figure 3. SB3 thermocouple layout
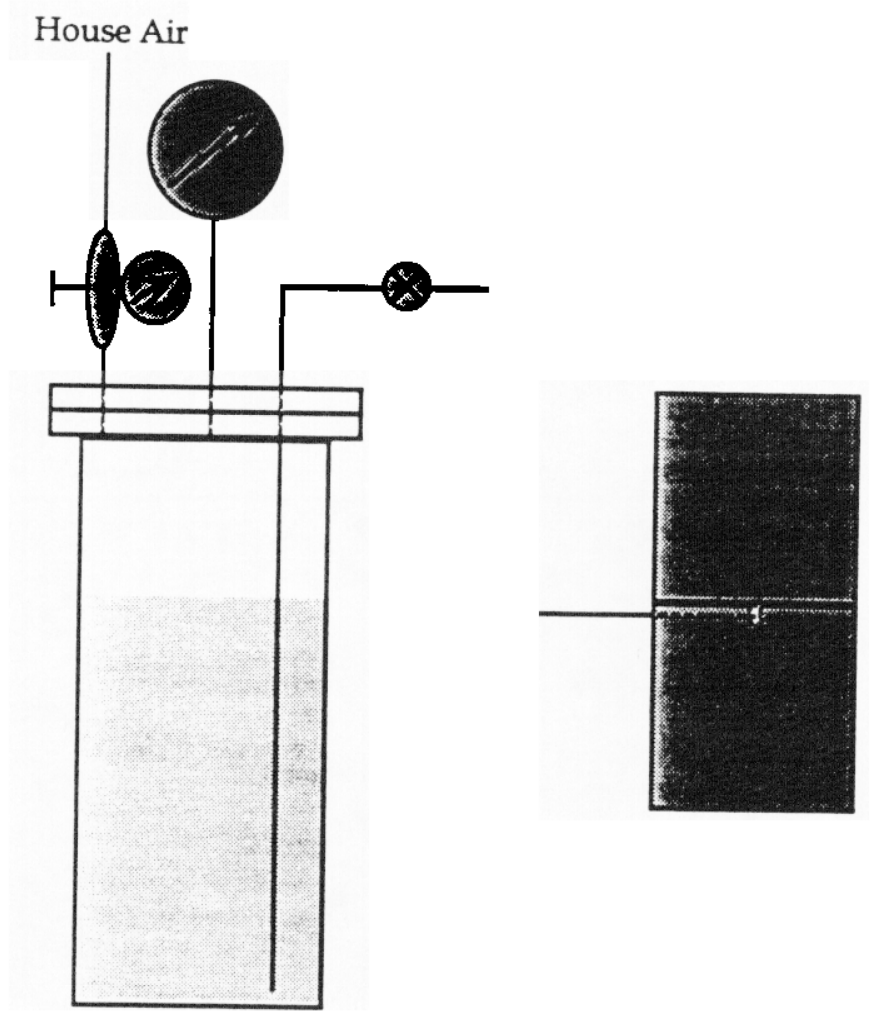

Figure 4. SB3 Fluid Flow System. The source cavity is about $2 \times 6 \times 10 \mathrm{~mm}^{3}$, centered in the upper face of the bottom block. 


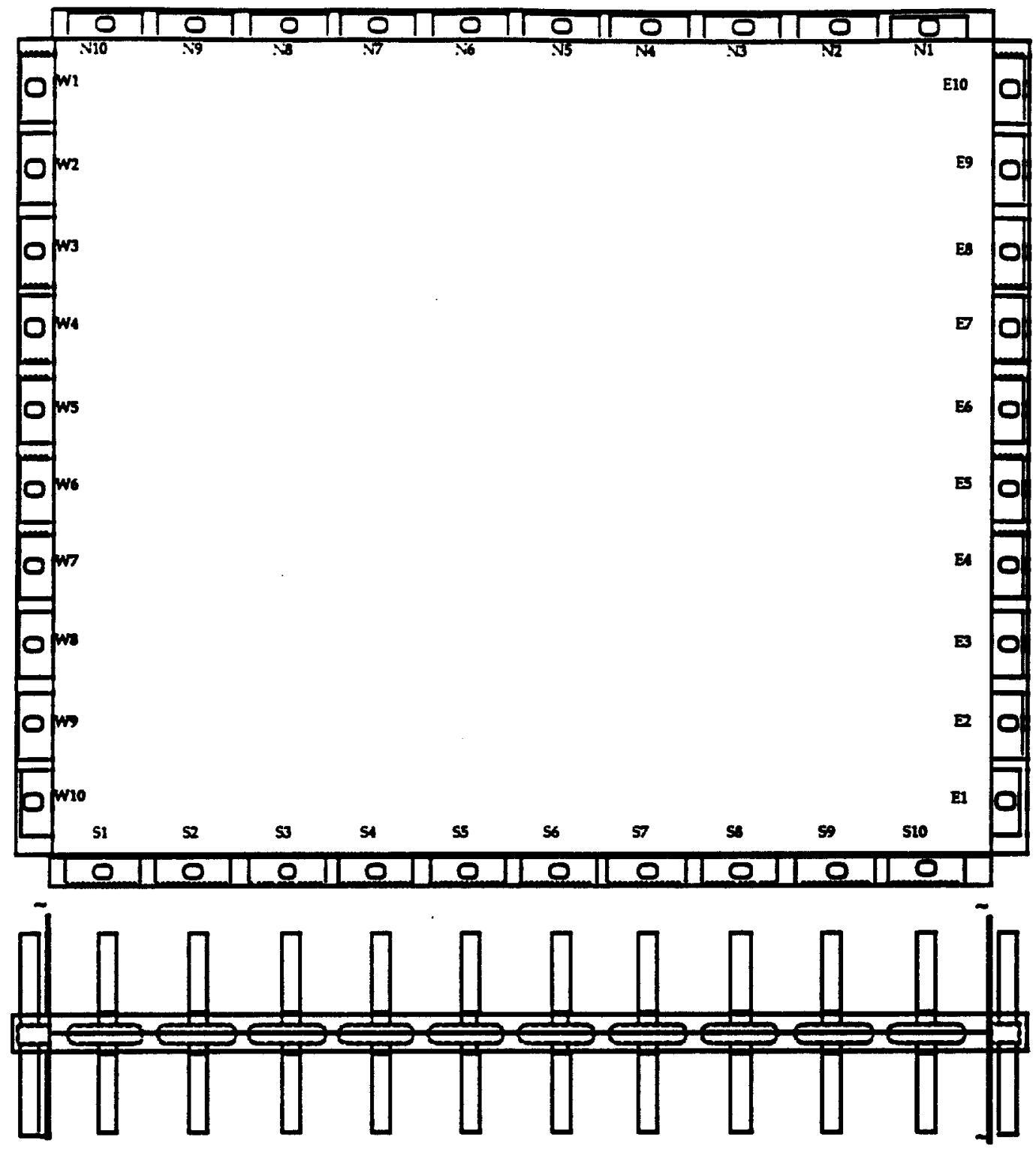

Figure 5. SB3 fluid collection manifold 
laboratory compressed air. The fluid is introduced as a point source by means of a .062" outer diameter x .044" inner diameter stainless steel tubing grouted into a .125" hole in the bottom block parallel to and about $1 \mathrm{~cm}$ below the fracture (Blair and Costantino, 1997). The fluid is collected along the perimeter of the crack using a simple manifold (see Figure 5) made of brass with copper and PVC tubing. Flow through the fracture at each face is collected into ten equally spaced ports about $20 \mathrm{~mm}$ long, separated by about $4 \mathrm{~mm}$. (The exceptions are N3 and N8, as they are covered by LVDTs 3 \& 5 and so were blocked off.) The manifold is mounted to the blocks using Dow Coming 739 RTV Sealant, which also ensures there is no flow between the ports. A length of PVC tubing carries the fluid to a collection container for that port, and the container is weighed to determine the amount of fluid exiting the fracture at the port (Blair and Costantino, 1997). This information is then entered into a Microsoft Excel spreadsheet program which can subsequently graph the fluid flow with the help of KaleidaGraph by Synergy Software. The amount of fluid into the block is determined by a glass tube connected parallel and down the length of the reservoir, lined by a tape measure. Displacement of water level noted on the tape measure, multiplied by a known constant for the cylindrical reservoir, immediately gives us the amount of flow into the block.

Standard procedure so far is to start at $0 \mathrm{MPa}$ vertical (or axial) stress, then test fluid flow into and out of the block at a driving pressure of 2 psi until graphs of both lines for in- and out-flow appear linear and parallel over time, then increase driving pressure to 3 psi, take data until linear and parallel again, increase to 5 psi, and finally go to 6 psi. Then increase axial stress to $.5 \mathrm{MPa}$ and start at 2 psi again, repeat the above process, go to 1 $\mathrm{MPa}, 2 \mathrm{MPa}, 4 \mathrm{MPa}$, and finally $8 \mathrm{MPa}$. Then go down to 4, 2, 1, .5, and $0 \mathrm{MPa}$ again, still starting with 2 psi, and see how things have changed.

My personal contributions to this test have included (but are not limited to!) clearing out the RTV coating from the holes in the fracture of the block, helping to mount the 
Figure 6.

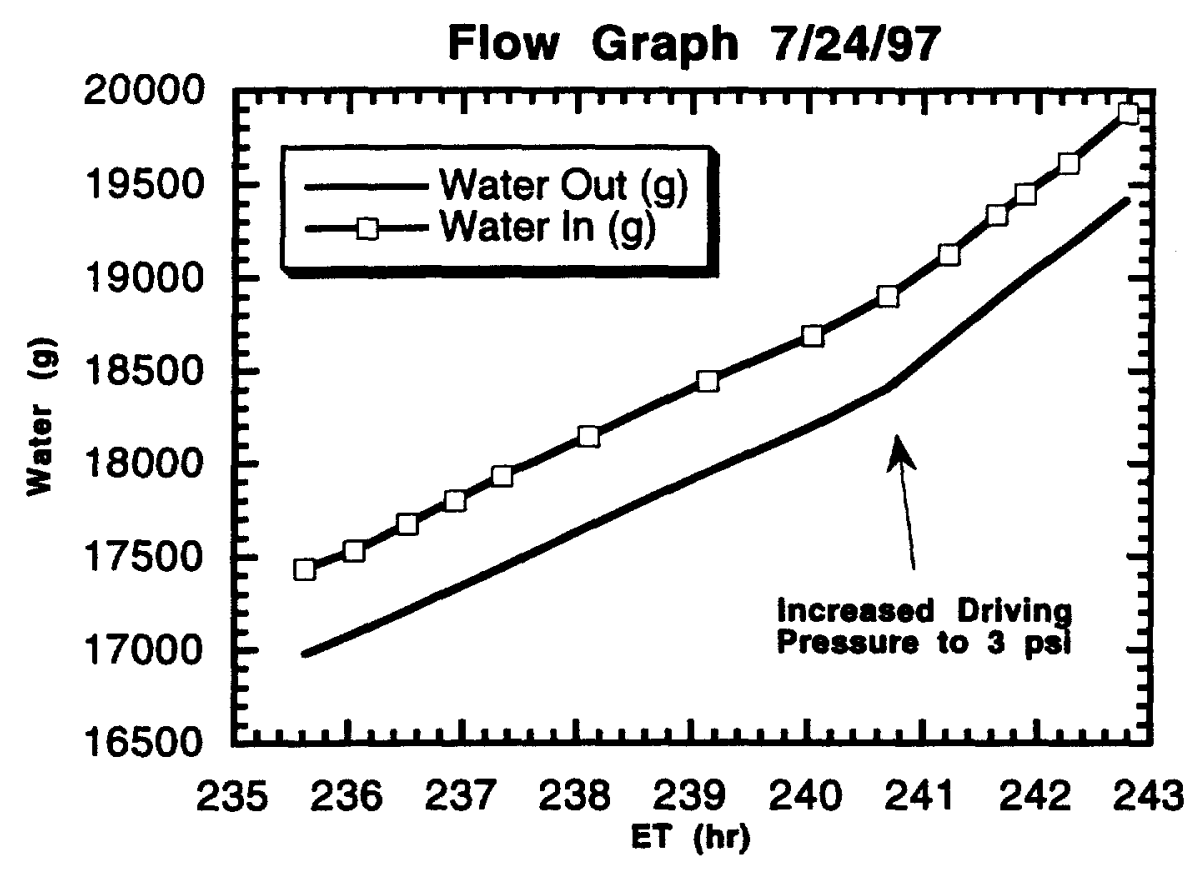

manifold as well as the LVDTs onto the block, helping to calibrate the type J thermocouples by dipping them in ice water, developing an Excel/KaleidaGraph spreadsheet program to input and graph flow data, monitoring the running experiment, and driving supervisor Marc Costantino slowly but surely insane.

\section{Results and Discussion}

As of the writing of this report, the experiment has been running for about three weeks, and will most likely continue to run for at least another few weeks. So far, the curves for water into and out of the block seem consistently parallel. (See Figure 6.) Changing the driving pressure on the reservoir just changes the Water In and Water Out curves almost instantaneously, but they still remain parallel. This indicates that not much water has been absorbed into the block, with most of the displacement between the in- and out-flow curves occurring early in the experiment. 
As the experiment proceeded, when most of the water was flowing through one or two particular tubes, we closed them off to see where the flow would go next. This allowed us to learn something about flow mapping across the fracture plane. No matter the driving pressure or axial stress, S5 consistently received the most water, behind which W6 and S3 usually followed. After these tubes were closed off E5, E7, and S4 usually followed. N7 rarely had a lot of flow, but it almost always had at least a slow, steady stream. Water has entered most tubes so far at one point or another, with notable exceptions being S6 through S9, and S10 until recently.

\section{Conclusion and Future Work}

Yucca Mountain could be the solution to the ever-growing problem in America of nuclear waste, but it must be properly tested first. The small block tests have already, and will hopefully continue to increase our understanding of the coupled processes involved in waste burial. In the latest test, we have so far noted that at room temperature the rock does not saturate very quickly, if it ever will around a flat, horizontal fracture. We've also seen that direction of fluid flow out of this type of fracture cannot be predicted by expecting moderate flow near the location of a large flow. It is completely determined by the maze of tiny rock formations inside the fracture, along with the coupled processes mentioned above.

In the coming weeks, we plan on heating the block from heaters sitting on its top surface and seeing how water flow reacts to temperatures just under $100^{\circ} \mathrm{C}$. We are also interested in making stress-strain curves when we receive more data, and finally graphs similar to what is seen in Figure 1 after the experiment is finished running.

In conclusion, I would like to state that I've thoroughly enjoyed my time at LLNL, and believe that the internship program here is extremely valuable for anyone wanting to learn what a career in the sciences actually entails. Thanks to all who have made this experience possible. 
References:

Anderson, M.P. and W.W. Woessner. Applied Groundwater Modeling. Academic Press, 1992. p.331.

Blair, S.C. and M.S. Costantino. Preliminary Results of a Coupled Fracture-Flow Test at the 0.5m scale. Lawrence Livermore National Laboratory, May 1997. pp. 6, 15-17, Figures 3-6.

United States Energy INFOcard (1995). Energy Information Administration, U.S. Department of Energy. Last modified: May 30, 1996.

http://www.eia.doe.gov/neic/infocard.html

Whipple, C.G. Can nuclear waste be stored safely at Yucca Mountain? Scientific American, June 1996. pp. 72-79. 
JOINT INVERSION

OF GEOPHYSICAL DATA FOR

SITE CHARACTERIZATION AND

RESTORATION MONITORING

\author{
Christen D. Rowe \\ Supervisor: Patricia Berge \\ T1477; R1136; L-219 \\ Lawrence Livermore National Laboratory \\ Livermore, California 94550
}

There are several ways of investigating the properties of a

contaminated ground site. Electrical resistivity measurements can be translated into information about the fluids present in the ground:

their composition, position, and quantity. Measurements of the ultrasonic, or seismic velocities in the ground can tell a lot about its structure on small and large scales.

Traditional methods of characterizing contaminated ground sites involve drilling cores or wells into the ground, a prohibitively expensive process which can actually increase the possibility of contaminant spread. The goal of the Joint Inversion project is to investigate the relationships between non-intrusive methods of data collection, electrical resistivity and seismic velocity. We hope to construct a computer code capable of predicting properties of a site 
from seismic and electrical data that previously could only be found using drilling methods.

Nearing the end of the first year of the project, we have begun our investigation along a few paths which we believe will eventually converge on a solution. This summer, I began to compile a comprehensive data base of properties of sediment mixtures from earlier published research. It includes such variables as pressure, compressional wave velocity, saturation, porosity, etc. for comparison and will be analyzed for relationships between properties. We are doing experiments in the laboratory to confirm the data and augment the database. We have devised apparatus for testing the seismic and electrical properties of sediment samples, collected and constructed a variety of samples, and begun to investigate relationships between their characteristics. In two years we will do field investigations, testing our hypotheses on polluted sites. The data we compile will be analyzed for relationships between properties: electrical conductivity and fluid saturation, compressional wave velocity and porosity. 
Compressional Wave Velocity vs. Porosity of Various Unconsolidated Sediment Mixtures

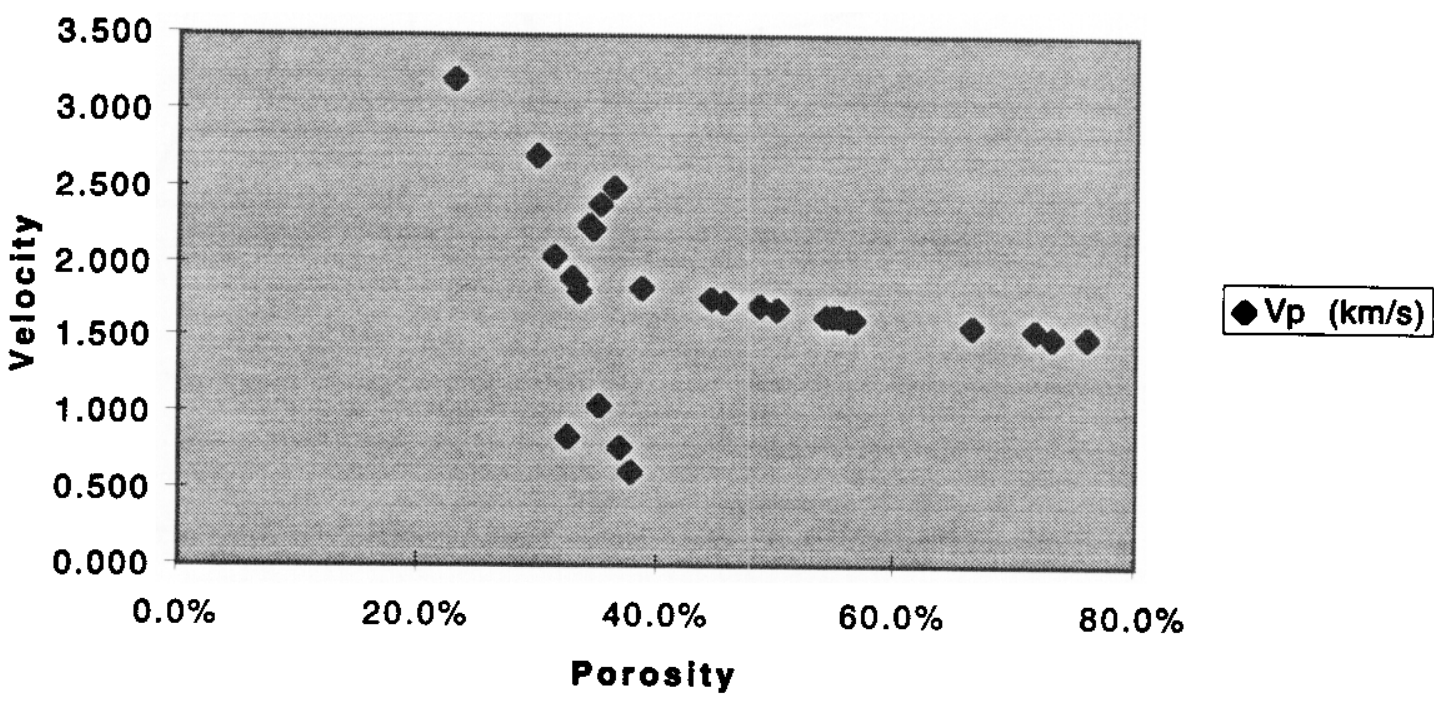

Fig. 1: The graph above shows a small sampling of the data we've collected from previous research, illustrating a possible relationship between two properties of soils we are investigating: porosity and compressional wave velocity. It also illustrates the difficulty in collecting data in this matter: stray points present a dilemma.

We may not have precise records of how experiments were performed, which makes it difficult to decide whether to discount this data as extraneous or investigate it as an important exception to the rule.

When the data collection stage of the project is completed, we will analyze the data to identify any existing relationships, such as the one implied by the graph above. When we have quantified the relationships, they will be programmed into a computer code.

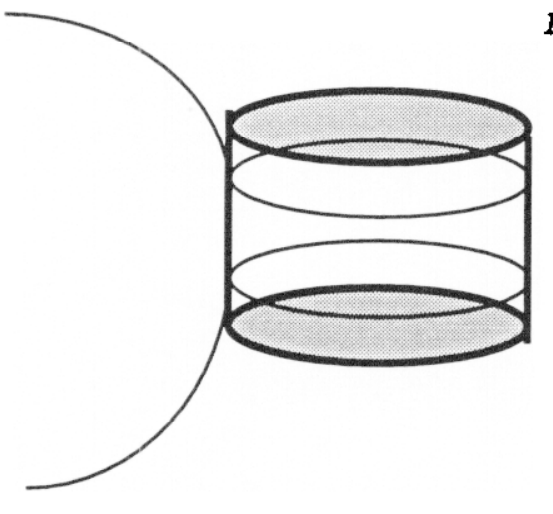

Fig. 2: Line drawing showing basic sample design. Gray ends of cylinder are metallic frits used for signal input for the four-electrode injection system. The inner circles on the cylinder are platinum-rhodium wire loops which act as the other set of electrodes to take measurements. 


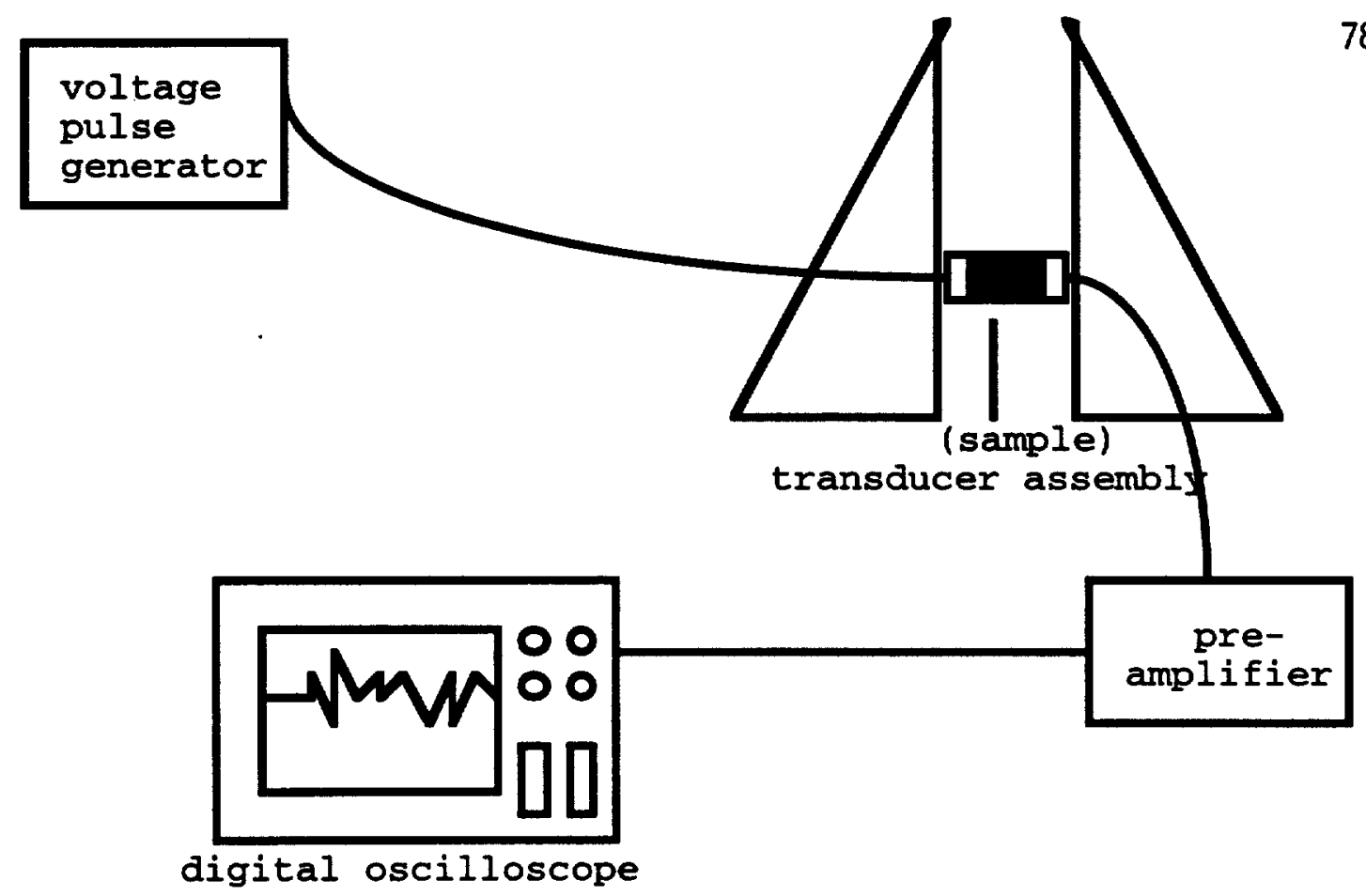

Fig. 3: Seismic testing apparatus. The voltage pulse generator releases a pulse of electricity, which is converted into an ultrasonic (compressional) wave by the transducers. It travels through the sample and is converted back to an electric signal on the other side. The travel time can be measured and velocity calculated very precisely by the oscilloscope. This assembly is very flexible in that it allows us to substitute a range of different transducers, and different types of sample designs.

Information on the progress and results of the Joint Inversion project will be available on the world wide web. As soon as it is posted, you can find it in a subdirectory under the LLNL Geosciences homepage at "http://www-ep.es.1lnl.gov/www-ep/esd.html". 


\title{
Radiocarbon Levels in Central and South San Francisco Bay
}

\author{
Maureen Ryan \\ University of South Carolina \\ Center for Accelerator Mass Spectrometry
}

The radioactive isotope ${ }^{14} \mathrm{C}$ occurs in nature, but over time anthropogenic events such as the industrial revolution and atomic testing have altered these natural levels. Measurements of ${ }^{14} \mathrm{C}$ levels in organisms can be used for a variety of applications in the natural sciences, including the monitoring of the bioavailability of petroleum-based pollutants. In order for such a study to be conducted, the naturally occurring levels of radiocarbon must be known.

By utilizing Accelerator Mass Spectrometry technology, a survey of the ${ }^{14} \mathrm{C}$ levels marine organisms of San Francisco Bay was conducted at 5 sites around the Bay. Organic and calcareous samples were collected, pretreated, converted to graphite targets, and the $\Delta^{14} \mathrm{C}$ levels determined through AMS.

Higher levels of $\Delta^{14} \mathrm{C}$ were observed for South Bay sites in comparison to Central Bay sites. These differences can most likely be attributed to decreased freshwater input, increased exposure to atmospheric $\mathrm{CO}_{2}$, and increased municipal waste input into the South Bay waters. The data produced correlates well with a previous study on $\Delta^{14} \mathrm{C}$ levels in water from the Bay.

$7 / 29 / 97$ 


\title{
Radiocarbon Levels in Central and South San Francisco Bay
}

\author{
Supervisor: Dr. Michaele Kashgarian \\ T2925; 112; L-397 \\ Lawrence Livermore National Laboratory \\ Livermore, California 94550
}

Introduction:

Radiocarbon $\left({ }^{14} \mathrm{C}\right)$ is produced naturally in the environment when cosmic rays interact with atoms in the atmosphere. Anthropogenic activities such as the industrial revolution and atomic testing have altered these natural levels. This atmospheric radiocarbon is then distributed into the oceans through gas exchange across the atmosphere-ocean interface. ${ }^{14} \mathrm{C}$ accumulates naturally in all living organisms through their metabolic pathways, and is usually reported as $\Delta^{14} \mathrm{C}$, which is the calculation in parts per thousand (\%o) of the ratio of ${ }^{14} \mathrm{C} /{ }^{12} \mathrm{C}$ found in the sample compared to the ${ }^{14} \mathrm{C} /{ }^{12} \mathrm{C}$ ratio of a standard.

A variety of factors such as the uptake of municipal waste or petroleum based pollution can influence these levels. Petroleumbased pollutants are "dead carbon," that is, they contain no radiocarbon and would therefore depress $\Delta^{14} \mathrm{C}$ levels in an organism. 
Municipal waste on the other hand has higher levels of $\Delta^{14} \mathrm{C}$ than normally found in water due to its increased exposure to atmospheric ${ }^{14} \mathrm{C}$, so the uptake of waste would raise $\Delta^{14} \mathrm{C}$ levels. Monitoring of these ${ }^{14} \mathrm{C}$ levels can be used in various applications in many of the natural sciences. One such application is the monitoring of the bioavailability of petroleum-based pollutants to organisms. In order for such a study to be successful, the naturally occurring levels of $\Delta^{14} \mathrm{C}$ in the organisms to be studied must be known.

The purpose of this study was to conduct a comprehensive study of the existing levels of $\Delta^{14} \mathrm{C}$ in marine organisms in located in Central and South San Francisco Bay by utilizing Accelerator Mass Spectrometry technology. Sites that were considered relatively pollution-free were chosen for study so that they could serve as a benchmark for future studies of polluted sites.

Materials and Methods:

Samples for analysis were collected from 5 separate sites around San Francisco Bay. One of the sites was accessed from the USGS $R V$ Polaris. Three of the sites were local beaches, Crown Beach, Point San Bruno, and Candlestick Point. The fifth site was Berkeley Pier. For the 
beach and pier sites, visible organisms were removed from solid substrates such as rocks, drainage pipes and pier pilings. Also, benthic organisms were sampled from sediments using a shovel and sieved through a series of USA Standard Sieve Series of mesh sizes: 10, 16, and 35. For the site sampled on the USGS cruise, a grab sampler was dropped from a winch over the side of the RV and the resulting sediment was sieved through box sieves. Two separate grabs were taken in this manner. All organisms were placed in plastic bags and put into a cooler containing dry ice immediately after collection.

All shells were pretreated by leaching with $0.1 \mathrm{~N} \mathrm{HCl}$. The organic samples were pretreated with $0.1 \mathrm{~N} \mathrm{HCl}$ acid baths until clean, followed by 2 washes with DI water. All samples were dried overnight on heating blocks. Approximately $10 \mathrm{mg}$ of each of the shells was placed in blood vials and converted to $\mathrm{CO}_{2}$ by placing the vials under vacuum and dissolving the shells with phosphoric acid. For organic samples, 3-10 mg of each of the samples was placed in $6 \mathrm{~mm}$ quartz combustion tubes. The equivalent of approximately 20 times the sample weight of $\mathrm{CuO}$ was added to the tube. The tubes were heat sealed under vacuum and combusted at $900^{\circ} \mathrm{C}$ for 3.5 hours. The resulting $\mathrm{CO}_{2}$ gas was converted to graphite using a cobalt catalyst reduction line where the $\mathrm{CO}_{2}$ gas is heated at $570^{\circ} \mathrm{C}$ with 2.1 times the $\mathrm{CO}_{2}$ volume of $\mathrm{H}_{2}$. Graphite is produced on the cobalt catalyst with 
water as a reaction by-product. The water is frozen out the sample using cold traps and the graphite is pounded into aluminum targets for AMS analysis (1).

Using AMS, the ratio of ${ }^{14} \mathrm{C} /{ }^{12} \mathrm{C}$ is calculated in relation to an NIST Oxalic Acid 1 standard. The NIST Ox-1 standard's $\Delta^{14} \mathrm{C}$ activity is equivalent to wood grown before the industrial revolution (1890), therefore serving as a zero mark for comparison since it shows only the natural levels of ${ }^{14} \mathrm{C}$ occurring in the atmosphere. $\Delta^{14} \mathrm{C}(\%)$ is determined using the definition:

$\Delta^{14} \mathrm{C}(\%)=\left[\left({ }^{14} \mathrm{C} / \mathrm{C}_{\text {sample }}{ }^{14} \mathrm{C} / \mathrm{C}_{\text {standard }}\right) /\left({ }^{14} \mathrm{C} / \mathrm{C}_{\text {standard }}\right)\right] 1000$ and a correction is made to compensate for fractionation due to different ${ }^{13} \mathrm{C}$ contents of masses of separate samples. 
Results:

Table 1: $\Delta^{14} \mathrm{C}(\%$ ) levels Sample ID

\section{Grab 1}

Tube Worm (G1TW)

$\Delta^{14 C} \quad+/-$

(\%o)

Worm 1 (G1W1)

57.4

5.2

$75.9 \quad 6.0$

Worm 2 (G1W2)

53.6

5.0

Mini Clams (G1MC)

66.9

5.4

\section{Grab 2}

Worm (G2W)

Mini Clams (G2MC)

60.2

5.2

69.8

6.2

Candlestick Point

Seaweed (CPSW)

48.4

5.3

Invertebrates (CPIV)

67.3

5.4

Point San Bruno

Barnacle Tissue (PSBBG)

42.9

6.0

Barnacle Shell (PSBBS)

47.2

4.9

Snail Shell (PSBS)

Seaweed (PSBSW)

37.8

5.5

53.9

5.9

Mussel Tissue (PSBMT)

45.6

5.3

Mussel Shell (PSBMS)

38.0

5.9

Crown Beach

Barnacle Shell (CBBS)

29.5

6.9

Barnacle Tissue (CBBT)

39.9

6.8

68.7

43.1

Mussel Shell (CBMS)

Invertebrate (CB2IV)

57.7

7.2

7.4

39.4

4.8

Invertebrates (CB2IVS)

Seaweed 1 (CB2SW1)

Seaweed 2 (CB2SW2)

29.3

4.7

33.8

4.7

Berkeley Pier

4.7

Seaweed (BPSW)

Invertebrate (BPIV)

Invertebrates (BPIVS)

Mussel Shell (BPMS)

Barnacle Shell (BPBS)

Barnacle Tissue (BPBT)

29.8

6.0

21.4

6.7

8.4

5.7

31.8

5.3

30.6

7.6

9.1

Worm Tissue (BPWT)

12.2

5.7

5.8 


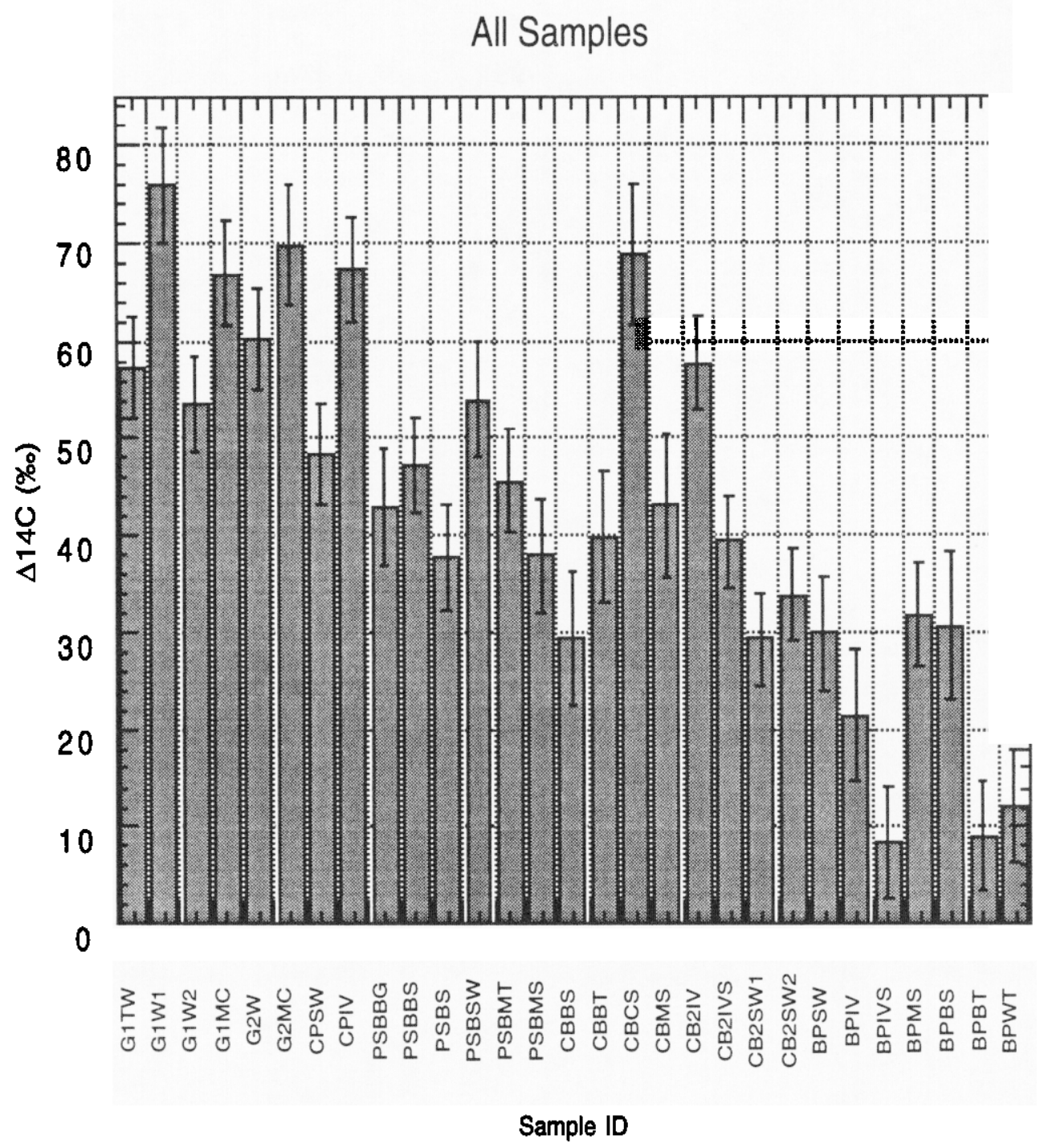

Figure 1: Sample ID vs. $\Delta^{14} \mathrm{C}(\%)$ for all samples collected. 


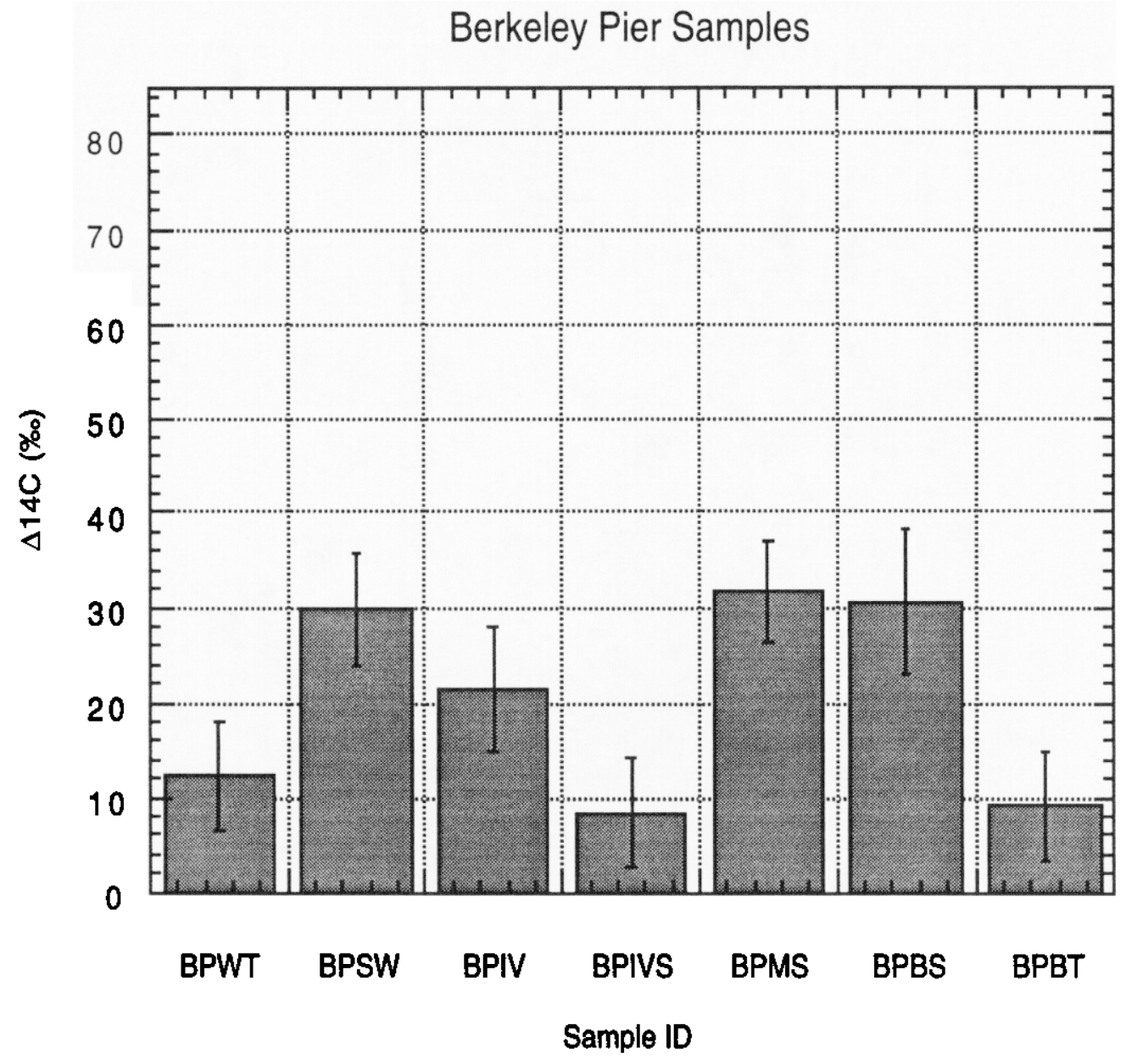

Figure 2: Sample ID vs. $\Delta^{14} \mathrm{C}(\% \circ)$ for Berkeley Pier Samples. 


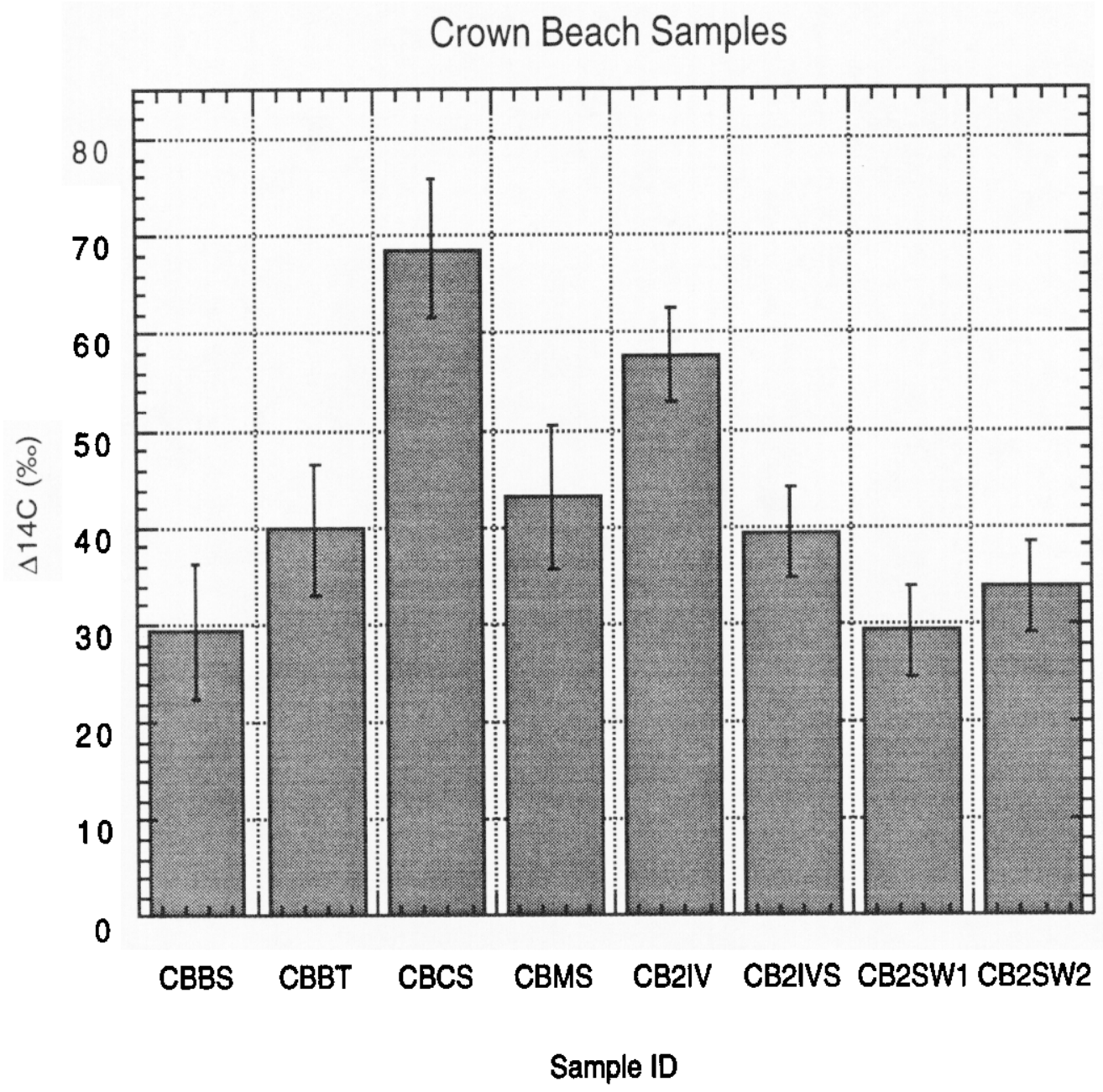

Figure 3: Sample ID vs. $\Delta^{14} \mathrm{C}(\%)$ for Crown Beach Samples. 
Candlestick Point Samples

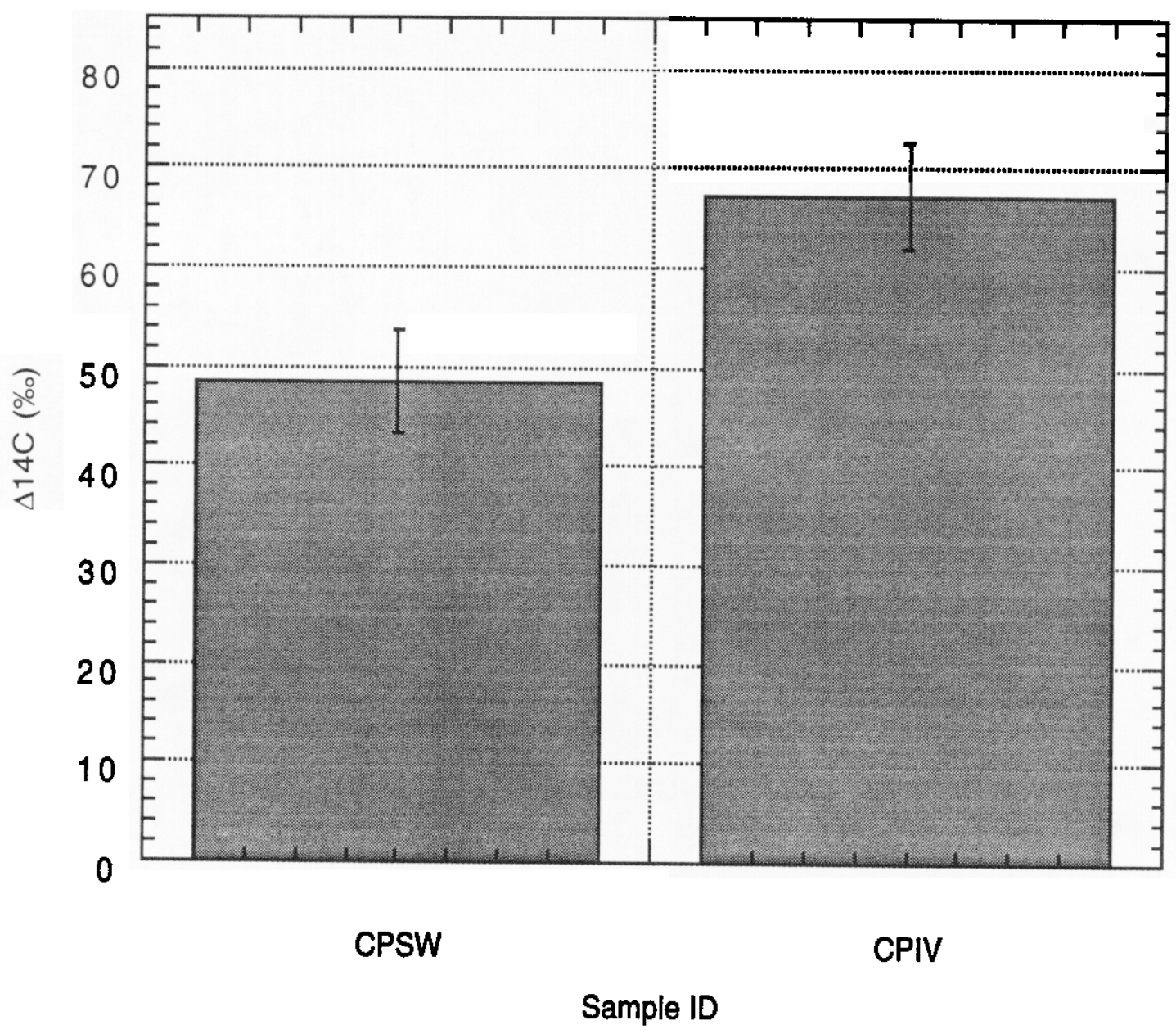

Figure 4: Sample ID vs. $\Delta^{14} \mathrm{C}(\% \circ)$ for Candlestick Point Samples. 


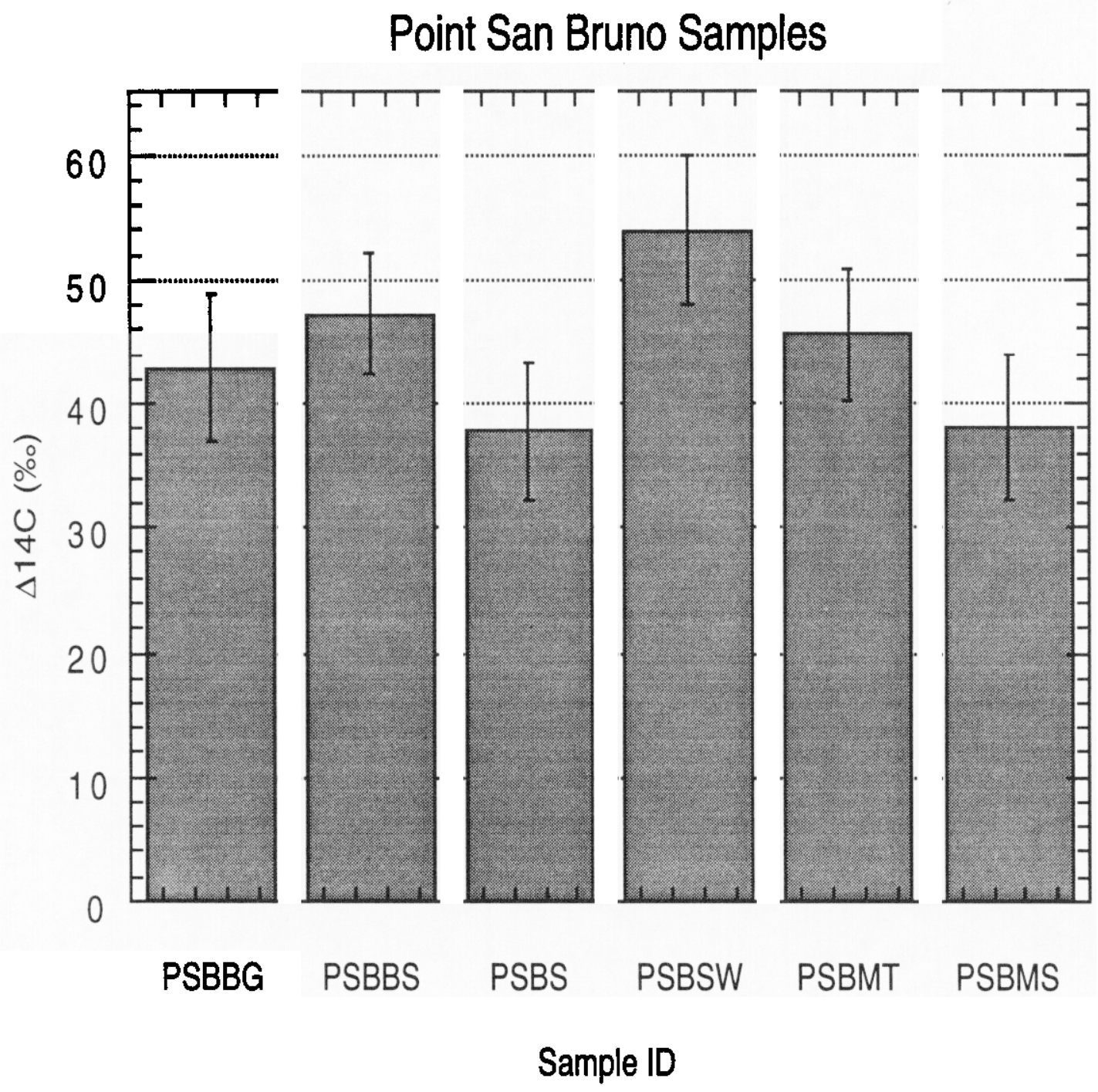

Figure 5: Sample ID vs. $\Delta^{14} \mathrm{C}(\%)$ for Point San Bruno Samples. 


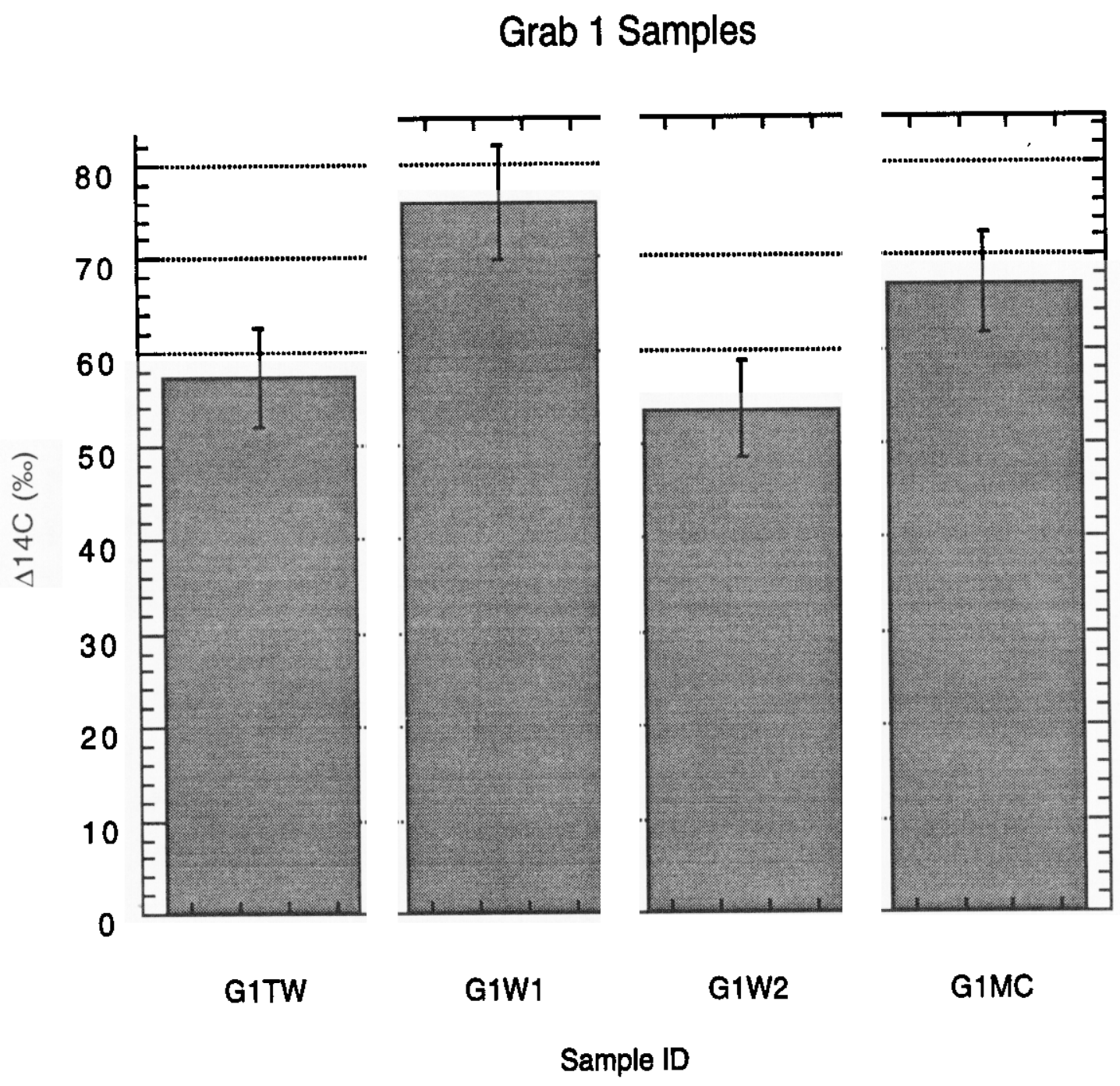

Figure 6: Sample ID vs. $\Delta^{14} \mathrm{C}(\% \circ)$ for Samples taken in Grab 1. 
Grab 2 Samples

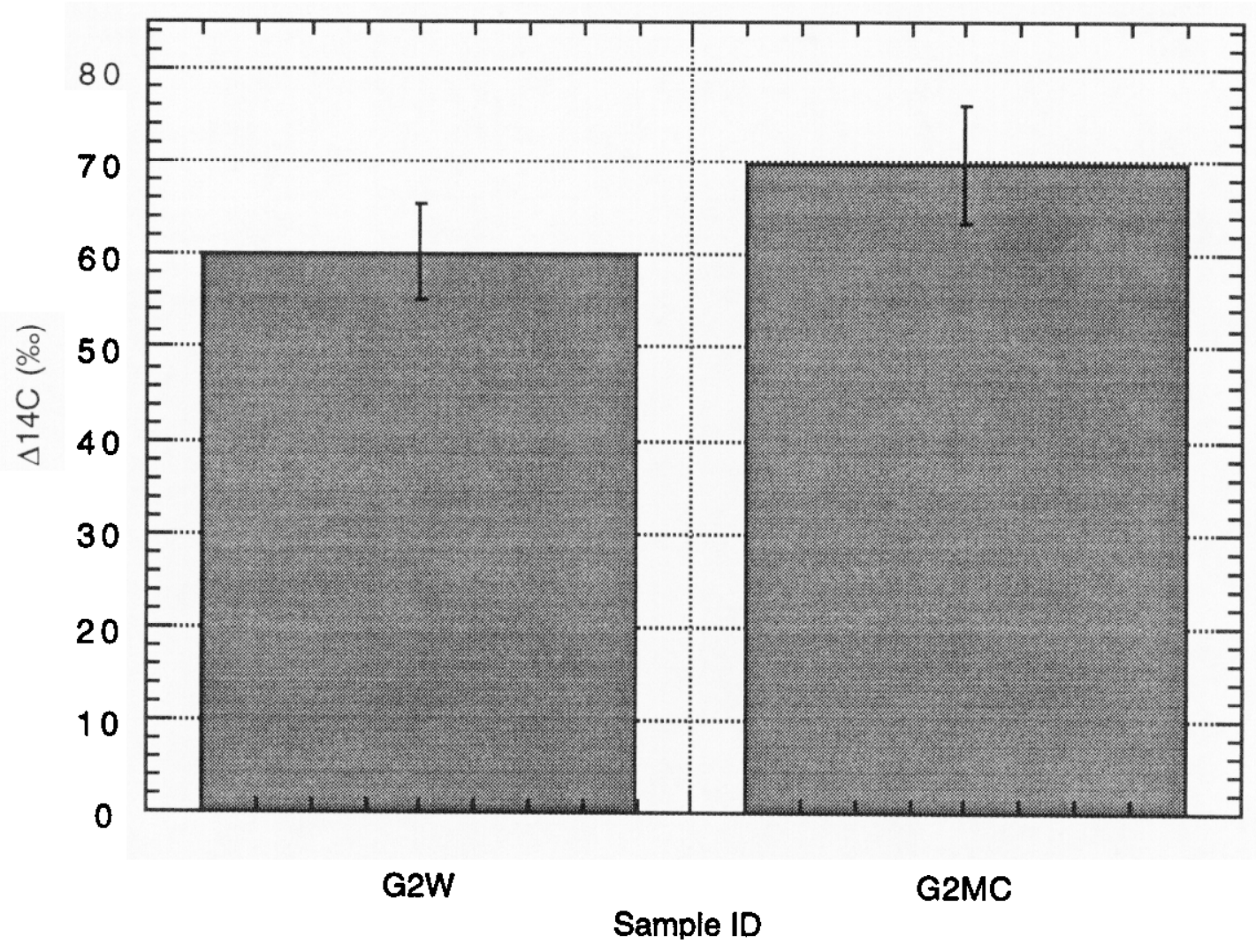

Figure 7: Sample ID vs. $\Delta^{14} \mathrm{C}(\%)$ for Samples take in Grab 2. 
Shells

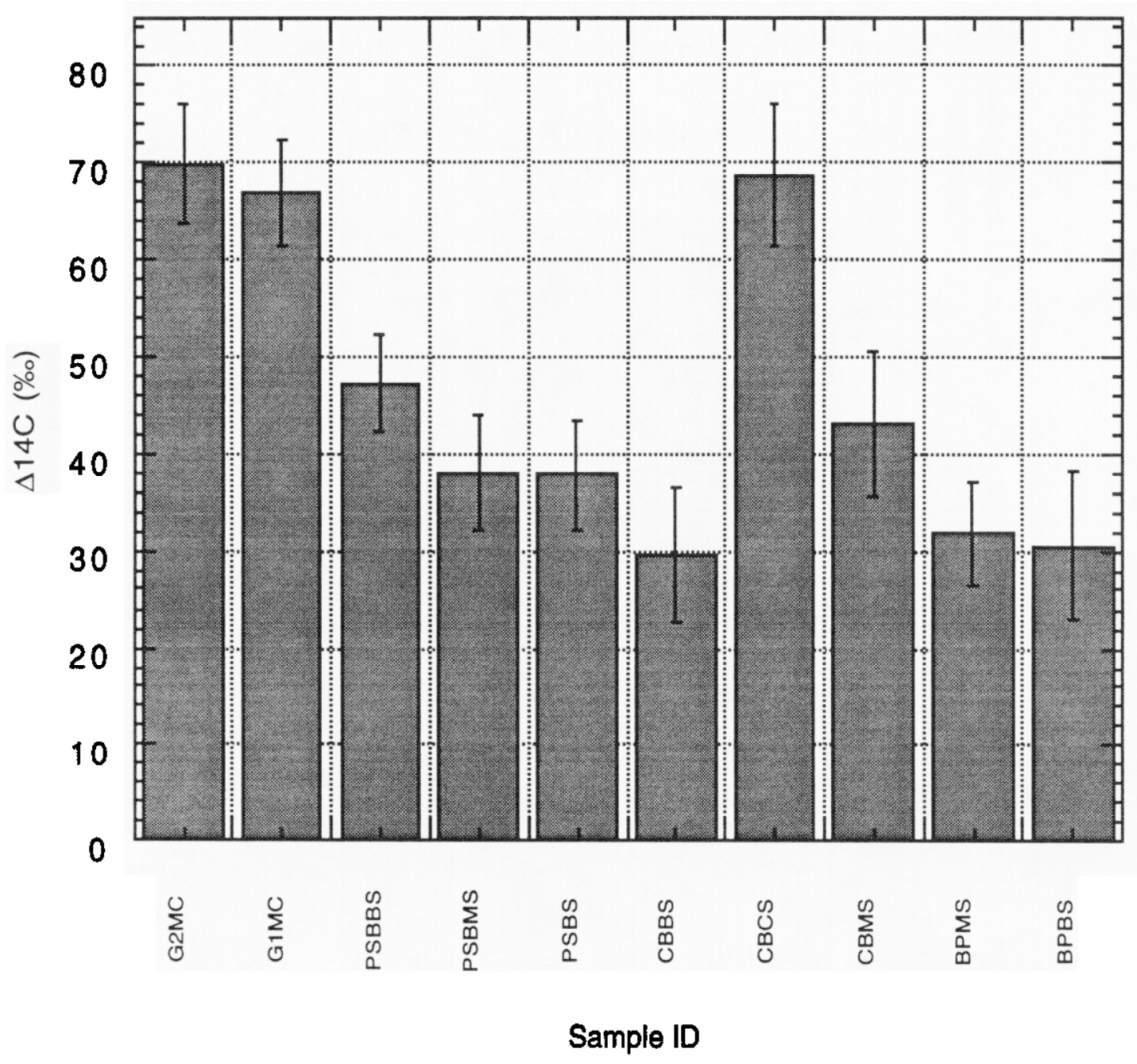

Figure 8: Sample ID vs. $\Delta^{14} \mathrm{C}(\%)$ for Shell Samples. 


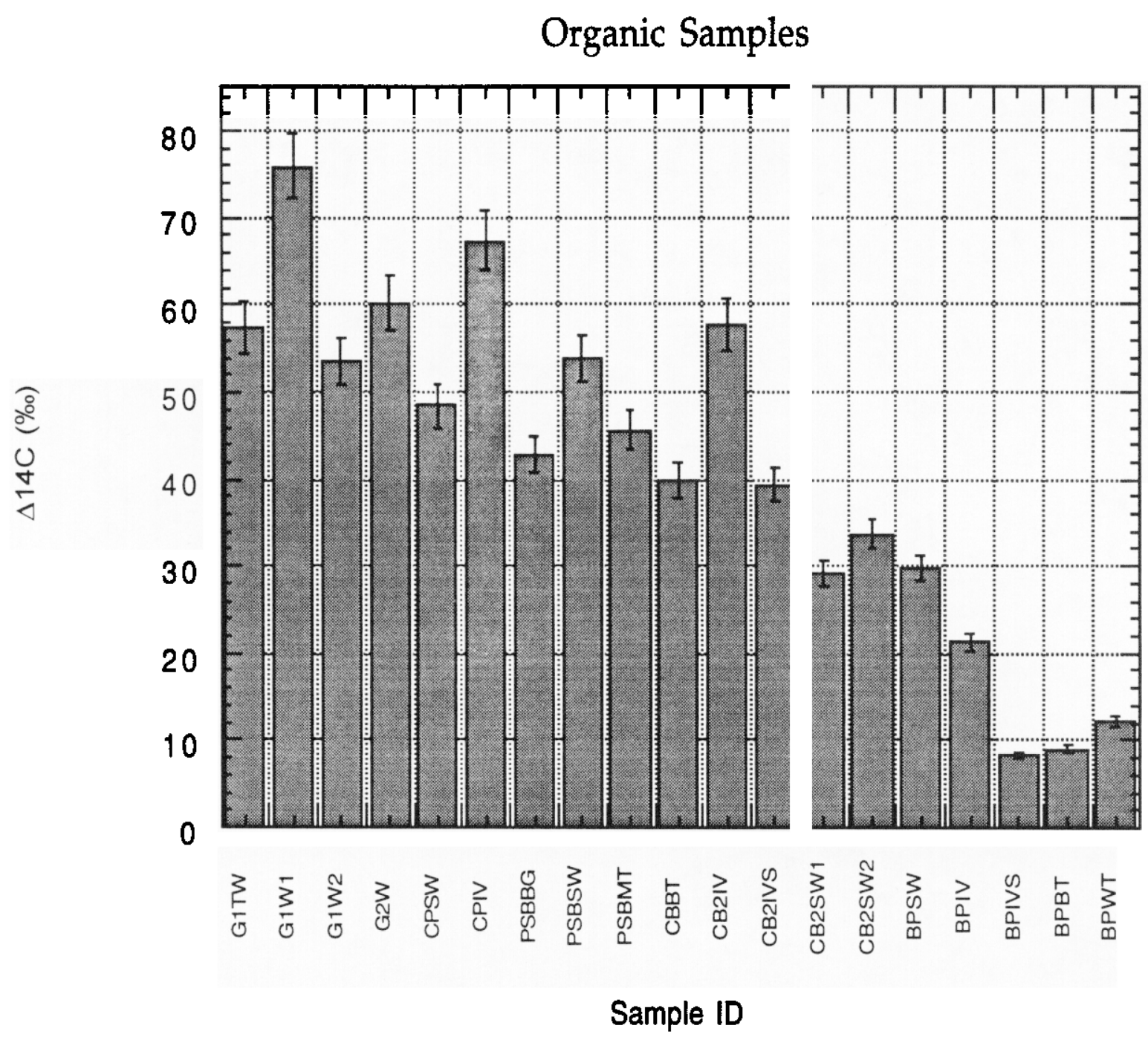

Figure 9: Sample ID vs. $\Delta^{14} \mathrm{C}(\%)$ for Organic Samples. 


\section{Sites Sampled}

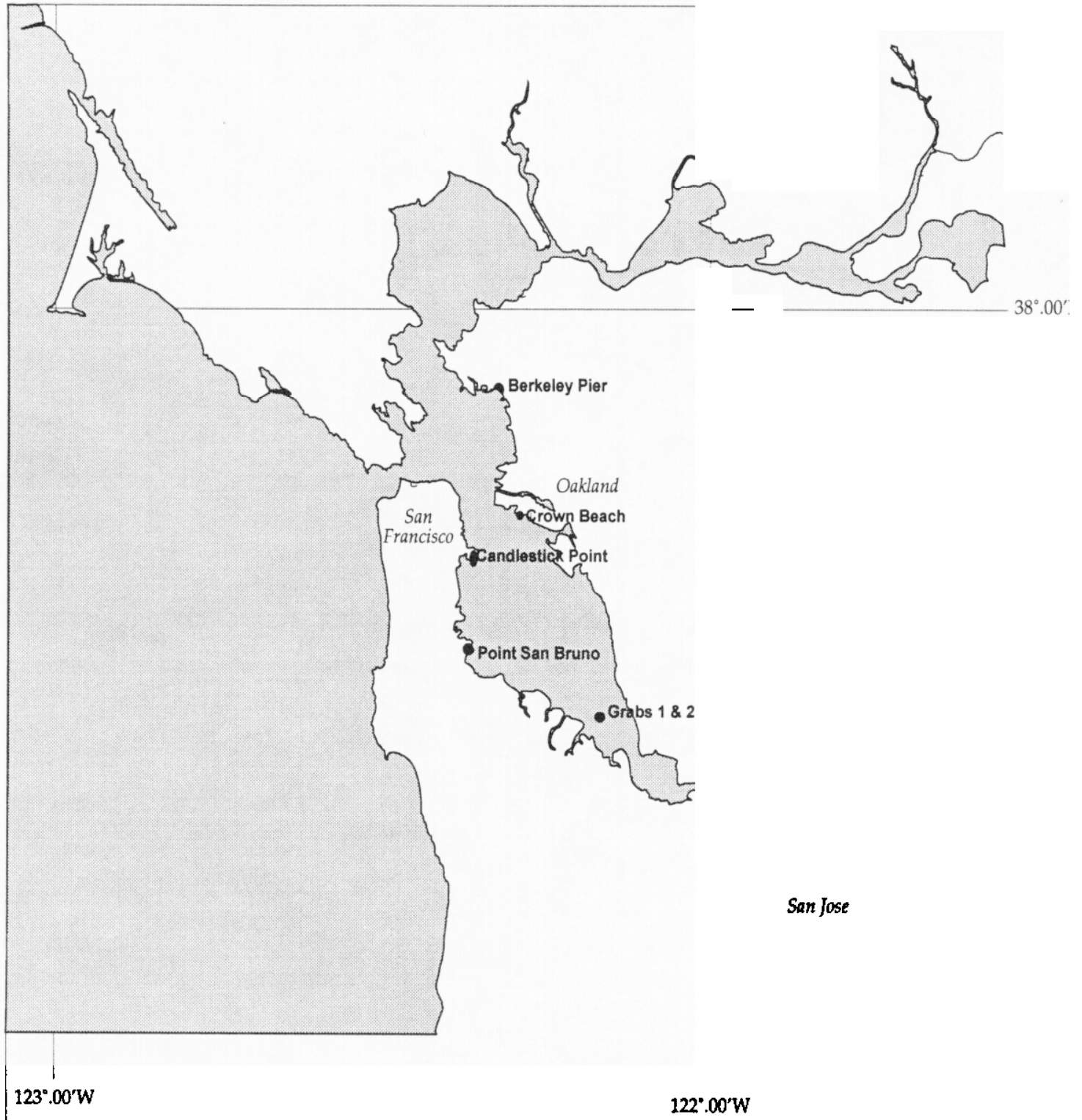

\section{San Francisco Bay Area}

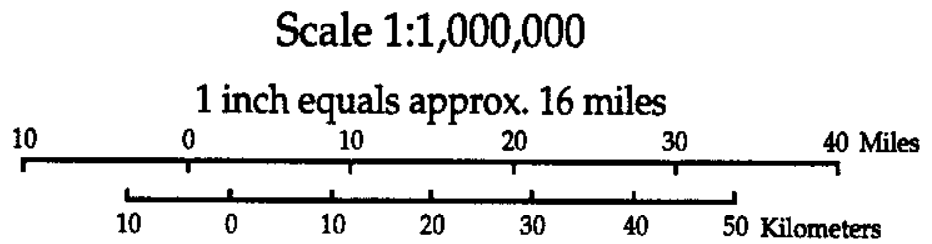


A general trend towards higher levels of $\Delta^{14} \mathrm{C}$ is shown moving from right to left on the $\mathrm{x}$-axis, correlating to the farther south the site was located (Figure 1). The lowest $\Delta^{14} \mathrm{C}$ levels (between $8.4 \%$ and $31.8 \%$ ) occurred at Berkeley Pier (Figure 2). The highest occurred at the USGS Grab sample sites (between $53.6 \%$ and $75.9 \%$ ) (Figures 6 \& 7). Organic tissue exhibited higher variations in $\Delta^{14} \mathrm{C}$ levels between samples than shells (Figures 8869 ).

Discussion:

San Francisco Bay is known to be an extremely complex estuarine system. There are many influences on carbon cycling in the Bay including freshwater inflow, mixing processes with the open ocean, exposure to atmospheric $\mathrm{CO}_{2}$, and the introduction of municipal waste into the water which can effect $\Delta^{14} \mathrm{C}$ levels in the waters, and thus the organisms round the Bay. Levels of $\Delta^{14} \mathrm{C}$ were consistently higher the farther South the site was located. This can best be explained by dividing the area studied into to two regions, the Central Bay and the South Bay.

The radiocarbon in the Central Bay region is effected mainly by freshwater runoff from the Delta region at the mouth of the Bay and 
tidal mixing. The $\Delta^{14} \mathrm{C}$ levels of lakes and rivers is usually lower than the $\Delta^{14} \mathrm{C}$ levels of the atmosphere, so mixing with river runoff will depress the $\Delta^{14} \mathrm{C}$ levels (2). Oceanic water entering the Bay through the opening at Golden Gate Bridge will be considerably depleted in ${ }^{14} \mathrm{C}$ because of the coastal upwelling that occurs off the California coast. Cold, nutrient rich water comes up from the bottom due to circulation patterns in the ocean, but this water tends to be very old and have low $\Delta^{14} \mathrm{C}$ levels. Mixing with this water in the Bay will also help to lower $\Delta^{14} \mathrm{C}$ levels in the Central Bay. In a previous study of the $\Delta^{14} \mathrm{C}$ in the waters of San Francisco Bay, Spiker (1980) found that $\Delta^{14} \mathrm{C}$ levels of the Central Bay were lower than the South Bay.

In his study, Spiker found that $\Delta^{14} \mathrm{C}$ increased with decreasing salinity, in the southward direction. The latest salinity data from the USGS and the data collected for this study agree with Spiker's findings. The South Bay region is a tidal lagoon type estuary (3). Is consists of flat shoals and narrow channels. Because of its shallow bathymetry and dependence on the tides for circulation, the water of the South Bay has greater exposure to the atmosphere and has a longer time equilibrate with atmospheric $\mathrm{CO}_{2}$ than water of the Central Bay does. Atmospheric $\mathrm{CO}_{2}$ is has higher levels of $\Delta^{14} \mathrm{C}$ than ocean water does, atmospheric exchange causes higher levels of $\Delta^{14} \mathrm{C}$. Other 
factors which increase $\Delta^{14} \mathrm{C}$ in the South Bay waters are the lack of freshwater and overabundance of waste water. Freshwater inflows into the South Bay region are approximately $1 / 300$ the of the volume of the whole system, while the total waste water discharge into the South Bay is about 10 times this amount (3). Since waste water usually has a much higher atmospheric component than pure water, its input into the water would raise $\Delta^{14} \mathrm{C}$ levels. It appears that increased municipal waste inputs have contributed to the higher levels of $\Delta^{14} \mathrm{C}$ in the South Bay. Spiker drew the same conclusions in his 1980 study.

The differences between the shells and organic tissue can most likely be attributed to the differences in their metabolic pathways. It is believed that approximately $50 \%$ of the carbon used by shells comes from dissolved inorganic carbon, while the other $50 \%$ is made up of metabolic carbon (4). The levels of $\Delta^{14} \mathrm{C}$ in the food that the organisms collected eat can be very different(4), thus causing the variation in the $\Delta^{14} \mathrm{C}$ levels of the organisms. Dissolved inorganic carbon (DIC) is reflective of oceanic water levels of $\Delta^{14} \mathrm{C}$, much lower than atmospheric, and its incorporation into the shell would help surpress the variability in $\Delta^{14} \mathrm{C}$ that is usually seen due to variation in the organisms' diet. 
Conclusion:

$\Delta{ }^{14} \mathrm{C}$ levels in marine organisms located in Central and South San Francisco Bay increase the farther South they are located. This variation in $\Delta^{14} \mathrm{C}$ can most likely be attributed to the fact that the Central Bay receives more freshwater runoff and greater tidal mixing than the South Bay does, and that the South Bay is affected by municipal waste input. The differences seen between shell samples and organic samples is most likely due to the shells ability to incorporate dissolved inorganic carbon into their shells..

Footnotes:

1. Vogel, J. S., Southon J. R., and Nelson D. E. 1987. "Catalyst and binder effects in the use of filamentous graphite for AMS". NIM Phys. Res. B29: 50-58.

2. Spiker, Elliott C. 1980 . "The Behavior of ${ }^{14} \mathrm{C}$ and ${ }^{13} \mathrm{C}$ in Estuarine Water: Effects of In Situ $\mathrm{CO}_{2}$ Production and Atmospheric Exchange." Radiocarbon, V.22, No. 3: 647-654.

3. Cheng, Ralph T., Casulli, Vincenzo and Gartner, Jeffery W. 1993. “Tidal, Residual, Intertidal Mudflat (TRIM) Model and its Applications to San Francisco Bay, California.” Estuarine, Coastal and Shelf Science. V.36: 235-280. 
4. Tanaka, Noriyuki, Monaghan, Marc C. and Rye, Danny M. 1986. "Contribution of metabolic carbon to mollusc and barnacle shell carbonate." Nature. V. 320: 520-523. 


\title{
Transcript Mapping of Chromosome 19: Localization of THOP1, FTL, and LU genes
}

\author{
Matthew Torres; Christa Prange; Greg Lennon \\ Supervisor: Greg Lennon, Ph.D. \\ Building 361, Room 1846, L-452
}

Human Genome Center, Biology and Biotechnology Research Program

Lawrence Livermore National Laboratory, Livermore, CA 94550

\section{Introduction}

Transcript Mapping with ESTs

Only $3 \%$ of the entire human genome is protein coding sequence (Brenner in Adams et al., 1991). Each of these protein templates, or genes, is fragmented by introns, which do not code for functional proteins. As proteins are the fundamental building blocks of an organism, they play a dominant role in the observable phenotype of an organism. Thus, locating the positions of the $3 \%$ coding sequence is a necessary and formidable, but not an impossible task.

The Human Genome Project is a Department of Energy and National Institute of Health sponsored effort to map and sequence the entire human genome with the objective of better understanding human biology and medicine. Two schools of thought exist on the matter: full genomic sequencing and transcript mapping (mapping the expressed or exon regions of a chromosome) (Adams et al., 1991). Proponents of full genomic sequencing argue that transcript mapping fails to provide genetic information on nonexpressed, gene regulatory regions of the genome. Proponents of transcript mapping argue that full genomic sequencing is, expensive, redundant, and uninformative as to the map position of human genes. Presently, the effort to fully sequence millions of base 
pairs of human genomic DNA (at a cost of $\sim \$ 0.79$ /base) (JGI proposal, 1997), is coupled by transcript mapping with ESTs.

Specifically, ESTs are cDNA clones derived from mRNA samples taken from various body tissues at different developmental stages of an organism's life (i.e. fetal, juvenile, adult). The 5' and 3' sequences of an individual clone is entered in the database for Expressed Sequence Tags (dbEST) and can easily be compared to any other DNA sequence on the Internet. Individual ESTs can thus be considered specific "gene tags", and may be used to map and sequence the coding regions of genes which are buried within millions of base pairs of extraneous DNA.

In an effort to increase the efficiency of transcript mapping, the Integrated Molecular Analysis of Genomes and their Expression (I.M.A.G.E.) consortium at LLNL, and the Washington University-Merck Corporation EST project revolutionized the mass production of ESTs (Lennon et al., 1996). With almost 1,000,000 clones having been arrayed, ESTs are now the world's largest source of human genes (url: http://wwwbio.llnl.gov/bbrp/image/image.html) .

\section{THOP 1, FTL, and LU}

THOP1 and FTL were picked as candidate genes for a mapping project because they had not been localized to the high-resolution contig map of chromosome 19 and because their complete coding sequence was known. Mapping of the LU gene was performed by a request from researcher M. J. Telen, M.D. at Duke University, and was not based on an EST sequence.

Thimet Oligopeptidase 1(THOP1) is a human endopeptidase (protease) which was initially thought to reside within the linkage region for the late-onset Alzheimer Disease AD2 locus (apoE) on chromosome 19q 13.3 (Meckelein et al., 1995). Knowing that Alzheimer's Disease is partially caused by the proteolytic cleavage of amyloid B peptide from its precursor, amyloid precursor protein, Meckelein (1995) indicated that 
THOP1 would be a likely candidate for a disease-relevant gene if linkage to an $\mathrm{AD}$ locus could be demonstrated. The following report, however, provides evidence on the contrary to Meckelein's conclusions. We have demonstrated that THOP1 localizes to 19p13.3 rather than 19q13.3 and thus cannot be linked to the AD2 locus apoE.

Ferritin is a universal iron-storage protein found in the cells of animals, plants, molds, and bacteria (Munro, 1990). Ferritin Light Chain (FTL) is one of two types of Ferritin protein subunits (Heavy $=21000 \mathrm{Da}$ and Light $=19000 \mathrm{Da}$ ) which are structurally interchangeable (Dorner et al., 1985). Previous research by Shull and Theil (1983) and Didsbury et al. (1986) indicates that Ferritin maintains cellular iron at nontoxic levels which is attributed to a need to protect DNA from degradation by iron and oxygen.

Lutheran Blood Group glycoprotein (LU) is a Red Blood Cell group antigen of the immunoglobulin superfamily of proteins and is developmentally regulated in human liver (Parsons et al., 1995). Recently, the LU gene was found to code for both the Lutheran blood group antigen and the B-cell adhesion molecule (B-CAM) epithelial cancer antigen (El Nemer et al., 1997). The two antigens differ by the length of their cytoplasmic tails and are formed by alternate splicing of the LU gene (El Nemer et al., 1997).

\section{Methods and Materials}

cDNA Isolation and Selection Criteria for THOP I, FTL, and LU

ESTs for THOP1 and FTL were generated by RT-PCR of mRNA isolated from human neuroepithelial cell tissue (Stratagene catalog\# 937231) and umbilical vein endothelial cell tissue (Stratagene catalog\# 937223), respectively. cDNAs were cloned into pBluescript SK- plasmid vectors and transformed into E. coli DH10B hosts. All cloning and partial sequencing of the ESTs was performed by the Integrated Molecular Analysis of Genomes and their Expression (I.M.A.G.E.) consortium and the WashUMerck EST Sequencing Project (Lennon et. al., 1996). 
The complete coding sequence for each gene was compared to partial sequences in dbEST using the Basic Local Alignment Search Tool (BLAST, url: http:/www.ncbi.nlm.nih.gov/) program on the internet. The best matching ESTs were picked as representative cDNA clones for each gene and were used to probe a genomic cosmid library of chromosome 19.

\section{Cosmid Libraries and Hybridization Technique}

Cosmid libraries specific to chromosome 19 were created from flow-sorted hamster-human somatic cell hybrids. Cosmid clones were spotted onto a nylon filter using the Hewlett-Packard ORCA as described by (Copeland, A and Lennon, G., 1994). The filters were pre-hybridized in a hybridization tube with prehyb solution at $2 \mathrm{ml} /$ filter and at $65 \mathrm{C}$ for one hour prior to the addition of 32-P labeled probe. To decrease the possibility of obtaining false-positive results, filters were blocked with a mixture of $50 \mathrm{uL}$ salmon sperm DNA, $20 \mathrm{uL}$ poly-U, $12 \mathrm{uL} \mathrm{KS-,} \mathrm{and} 10 \mathrm{uL}$ human COT DNA at $1 \mathrm{mg} / \mathrm{ml}$. The mixture was boiled for $\sim 5$ minutes and then transferred to the prehyb solution.

During the prehybridization step, ESTs for THOP1 and FTL were tagged with 32-P labeled dCTP incorporated into multiple PCR copies isolated by primers (T3/T7) which flank the cDNA insert. Radiolabeled probe was then added to the hybridization tube with the cosmid filters. Hybridization tubes were placed on a rotisserie and incubated at $65 \mathrm{C}$ for 24 hours. Following hybridization, filters were washed with low stringency wash solution for 15 minutes, twice, followed by a high stringency wash for 10 minutes. All washes happened at $65 \mathrm{C}$. After washing, filters were sandwiched between two sheets of saran-wrap and placed in a phosphoimager cartridge overnight. We used the STORM 860 imaging machine (Molecular Dynamics) to scan the 32-P exposed phosphocartridges. Filter images were analyzed with ImageQuaNT software (Molecular Dynamics). 
Verification of Hybridization Positives

Verification of hybridization positives was accomplished with sequence specific PCR. Primers were designed from the complete coding sequences of THOP1 and FTL using the Baylor College of Medicine (BCM) primer selection program on the internet. The program selects primers from a given sequence based on GC content and forward/reverse primer anti-complimentarity. Because primer selection was based on coding sequence rather than genomic sequence, primers were derived from distinct exon sequences (when they were known), and were derived from the first $200 \mathrm{bp}$ of a coding sequence (when exon/exon distinctions were not known). This precautionary measure increased the probability that the primers would be useful for amplifying genomic DNA Once chosen, the primers were ordered from Genset biotechnology corporation. Purified DNA from the positive cosmid clones were retrieved from the frozen stock at LLNL and tested by PCR amplification for the presence of the sequences specific to the ordered primers. All samples were run on $1 \%$ agarose gels at $100 \mathrm{~V}$.

Verified positive cosmid clones were located on the cosmid contig maps for chromosome 19 using the Browser program at LLNL. Confirmation of band position was achieved by locating the FISH mapped cosmid clone nearest to the verified positive.

\section{Mapping the LU gene}

A short 500 bp probe of the LU gene was donated by M. J. Telen, M.D. at Duke University's department of medicine, radiolabeled with Prime-It Random Label Kit (Stratagene) and exposed to cosmid filters of chromosome 19 using the previously noted procedure. Primers used for the verification of hybridization positives were also donated by Duke University and were based on genomic sequence of the LU gene. 


\section{RESULTS}

Figure $2 \mathrm{a}, \mathrm{b}$, and $\mathrm{c}$ shows the physical map location for each gene. The cosmid contig restriction fragment map for $L U$ is shown as an example of the high resolution map to which each gene was localized (Figure 2c).

The THOP1 probe hybridized to cosmid clone 20149 of contig 1206 on chromosome 19p13.3. This is in contradiction with the previous report that THOP1 was linked to the AD2 locus on 19q13.3. We confirmed these results by using the same sequence specific primers to amplify cosmids surrounding clone 20149. The primers amplified cosmid clone 33074 which is also on contig 1206 of chromosome 19p13.3 (Figure 1). Immediate neighbors of THOP1 include the Muellerian Duct Syndrome gene (AMH), and Acute Lymphoblastic Leukemia gene (TCF3) (Figure 2a).

The FTL probe was localized to two regions on chromosome 19,19p13.3 and 19q13.3, which suggests that it is a member of a gene family. The FTL probe positively identified cosmids 18858, 21213 of contig 313; and 20664 of contig 2039 for presence of the FTL gene. Immediate neighbors of FTL include luteinizing hormone B polypeptide (LHB), and human glycogen synthase 1 (GYS1) (Figures 2a and b). 
(Figure 1). Immediate neighbors of THOP1 include the Muellerian Duct Syndrome gene (AMH), and Acute Lymphoblastic Leukemia gene (TCF3) (Figure 2a).

The FTL probe was localized to two regions on chromosome 19,19p13.3 and 19q13.3, which suggests that it is a member of a gene family. The FTL probe positively identified cosmids 18858, 21213 of contig 313; and 20664 of contig 2039 for presence of the FTL gene. Immediate neighbors of FTL include luteinizing hormone B polypeptide (LHB), and human glycogen synthase 1 (GYS1) (Figures 2a and b).

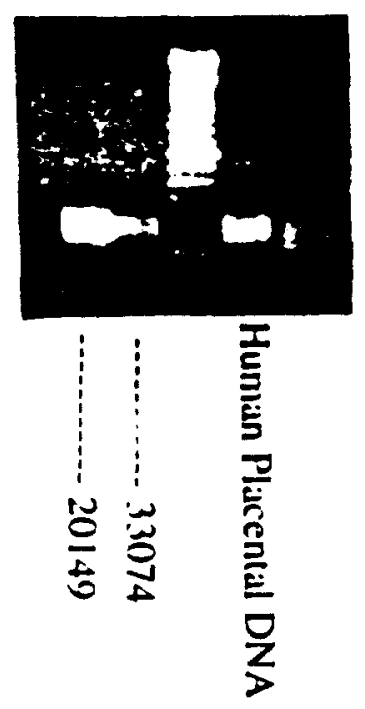

Figure 1: Gel electrophoresis of PCR amplified cosmids containing the THOP1 gene on chromosome 19p13.3. The amplified segment was $\sim 200$ base-pairs.

The LU probe was localized to chromosome 19q13.2 on cosmids $24639,21754,16252$, 5921 , and 29578 of contig 286. Immediate neighbors of LU include B-cell CLLAymphoma 3 (BCL3) and Apolipoprotein E (ApoE) (Figure 2c). All mapping information for these genes was downloaded onto the Browser program at LLNL. 

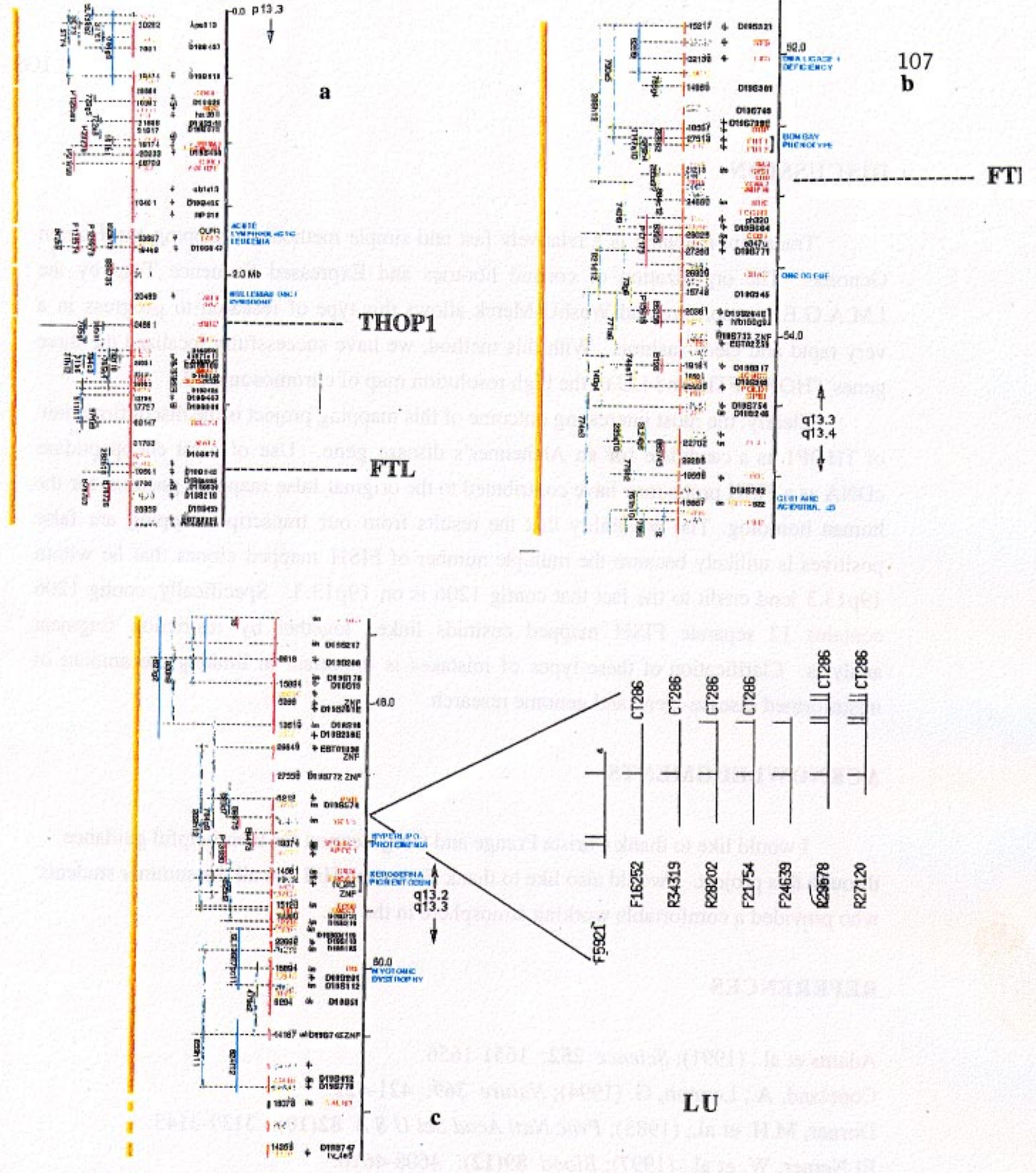

Figure 2: Physical maps of chromosome 19. (a) 19p13.3 including map positions for THOP1 and FTL. (b) 19q13.3 including map positions for FTL. (c) 19q13.2 including map positions for $\mathrm{LU}$ and also showing an example of the cosmid contig restriction fragment map which was identified with the sequence specific primers. 


\section{DISCUSSION}

Transcript mapping is a relatively fast and simple method of mapping the Human Genome. The organization of cosmid libraries and Expressed Sequence Tags by the I.M.A.G.E. consortium and WashU-Merck allows this type of research to progress in a very rapid and clean fashion. With this method, we have successfully localized the three genes THOP1, FTL, and LU to the high resolution map of chromosome 19.

Clearly, the most interesting outcome of this mapping project is the disconfirmation of THOP1 as a candidate for an Alzheimer's disease gene. Use of a rat endopeptidase cDNA as a FISH probe may have contributed to the original false mapping position for the human homolog. The possibility that the results from our transcript mapping are false positives is unlikely because the multiple number of FISH mapped clones that lie within 19p13.3 lend credit to the fact that contig 1206 is on 19p13.3. Specifically, contig 1206 contains 12 separate FISH mapped cosmids linked together by restriction fragment analysis. Clarification of these types of mistakes is important in limiting the amount of misinformed disease- gene and genome research.

\section{ACKNOWLEDGMENTS}

I would like to thank Christa Prange and Greg Lennon for their helpful guidance through this project. I would also like to thank Francesca Hill and all the summer students who provided a comfortable working atmosphere in the lab.

\section{REFERENCES}

Adams et al., (1991); Science 252: 1651-1656.

Copeland, A.; Lennon, G. (1994); Nature 369: 421-422.

Dorner, M.H. et al., (1985); Proc Natl Acad Sci U S A 82(10): 3139-3143.

El Nemer, W. et al., (1997); Blood 89(12): 4608-4616.

Lennon, G. et al., (1996); Genomics 33: 151-152.

Meckelein, B. et al., (1996); Genomics 31: 246-249. 


\section{Vacuum Control System for the \\ Production Laser Cutting Work Station}

Timm A. Wulff

Supervisor: Doug Sweeney

Building 691, Room 145

Lawrence Livermore National Laboratory

Livermore, California 94550

\section{Introduction}

Recent advances in laser technology at Lawrence Livermore National Laboratory have produced a femtosecond pulse laser. This laser has is currently designed to run at a nominal 15 watts and can cut holes 5 microns in size. This laser is being applied to a first of a kind Laser Cutting Work Station (LCWS) and is currently in production at Lawrence Livermore National Laboratory.

\section{Purpose}

Operations Control is the portion of the control system that the operators will interact with during the machining process. The vacuum control system is an application that is called on by Operations Control. The vacuum system provides the correct environment where cutting process is performed. The vacuum system is important because the quality of the cut is directly related to the degree of vacuum. The lower the pressure the faster the laser ejected material, plasma, is removed from the cut hole. Any plasma the is allowed to cool in the path deposits residue on the walls of the cut thus diminishing cut quality. 


\section{Problem}

The manipulation of the vacuum system occurs twice during a cut process. Once after the target has been placed in the vessel and the machine has been properly prepared, and before a cut begins. It is at this time that the vacuum vessel is 'Pumped Down' to a pressure less than $10^{-4}$ Torr. The second time the vacuum system is operated it is used to return the chamber to ambient pressure, 'Bleed Up' the system, once a cut has been completed.

The vacuum system has a current configuration that is comprised of 12 valves, a mechanical pump, a turbine pump, and 4 vacuum pressure sensors. (figure 1) Manually operating the valves and pumps during a pump down or a bleed up process involves a number of sequential steps. A complicated and routine process of valve operations leaves an opportunity for user error. Automating the process ensures that the system is operated in an approved manner.

However, during the time of developing the system the sequence of valve and pump manipulation had not been finalized. Also the number of devices requiring controls is subject to change. New equipment can and has been added to the vacuum system since work on the vacuum control system began. The system needed to be developed flexible enough to accept these changes.

\section{Solution}

My assignment was to develop the vacuum control system and user interface. Achieving control of the system was done using National Instruments LabVIEW (a visual programming language). 
LabVIEW also provides the user interface controls that the LCWS operators will use as part of the vacuum control. The code that operates the system was designed to follow guidelines described in the LCWS Graphical User Interface Guidelines as well as LCWS Software Implementation Guidelines.

The software that provides the controls and user interface for the vacuum control system were achieved using a National Instruments product called LabVIEW. LabVIEW executes its operations using blocks of code called Virtual Instruments (VIs).

The number of VIs called by the vacuum system were kept as low as possible. A simple hierarchy of VIs makes tracking data flow easier.

The VIs are designed to be flexible enough to be used to pump down and bleed up the system. The only difference the user sees between Pump Down and Bleed Up is the text that appears on the Auto Sequencing control button. During Pump Down the control button reads 'Press to Begin Pump Down,' and during Bleed Up the control read 'Press to Begin Bleed Up.'

The operator interface allows the user to give one command to perform pump down or bleed up. However, if a system error occurs or if the user chooses to do so the system may be operated manually. The automation also reduces the time required to reach the end of a pumping sequence.

Changing the order of sequencing of valve and pumps only requires the user to change a value in a spreadsheet that is read by the VI during a run. This spreadsheet that is read bv the VIs also

sequencing of pump down and bleed up pumping sequences was built but never completely tested. Testing was possible during the 
were the status of the valve can be read and where to write to change the valves condition.

Adding new devices to the vacuum system requires adding a new device to the spreadsheet. The software changes are minimal. This will be discussed latter in the Operations section of the paper.

The VIs, during operation, communicate the users requests via a RS232 communication line to a Programmable Logic Controller (PLC). The PLC checks the request to actuate a device against safety interlocks. Provided no safety interlocks exist on the device in the systems current configuration, the PLC delivers the power to operate the device. Should an interlock exist, the command is ignored.

The PLC also reads the current status from the valves, pumps and gages. This information is placed at specific location in memory where it can read by the running VI. The VI parses the pertinent status bits and prompts the user of the current status of the vacuum system. The current status of every device in the vacuum system determines which devices are interlocked.

\section{Conclusion}

The vacuum control system is currently being tested. The LCWS interface developed allows the user to control all the system valves, mechanical pump, and turbine pump. The system also displays the current state of all the vacuum system components. The manually controlled operation of the vacuum control system works very well.

The need for the system to be flexible to accommodate a growing family of controlled devices was also achieved. An auto 
sequencing of pump down and bleed up pumping sequences was built but never completely tested. Testing was possible during the term of my stay due to time constraints caused by mechanical hardware issues. 


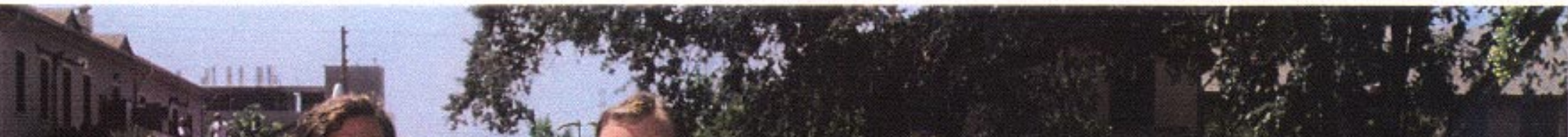

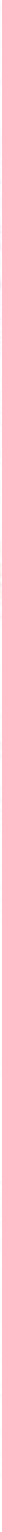


$100=6$

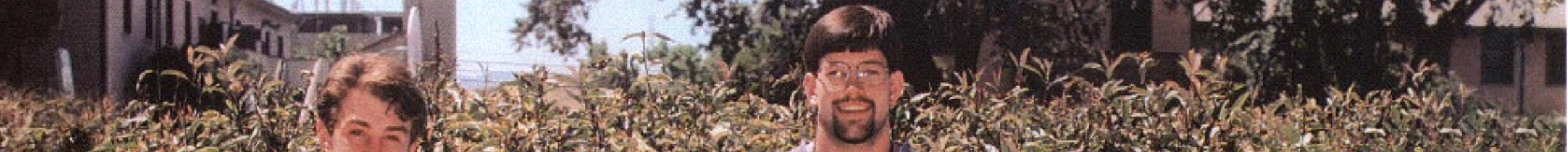

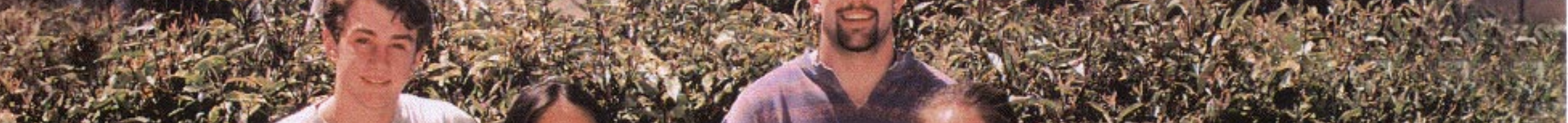

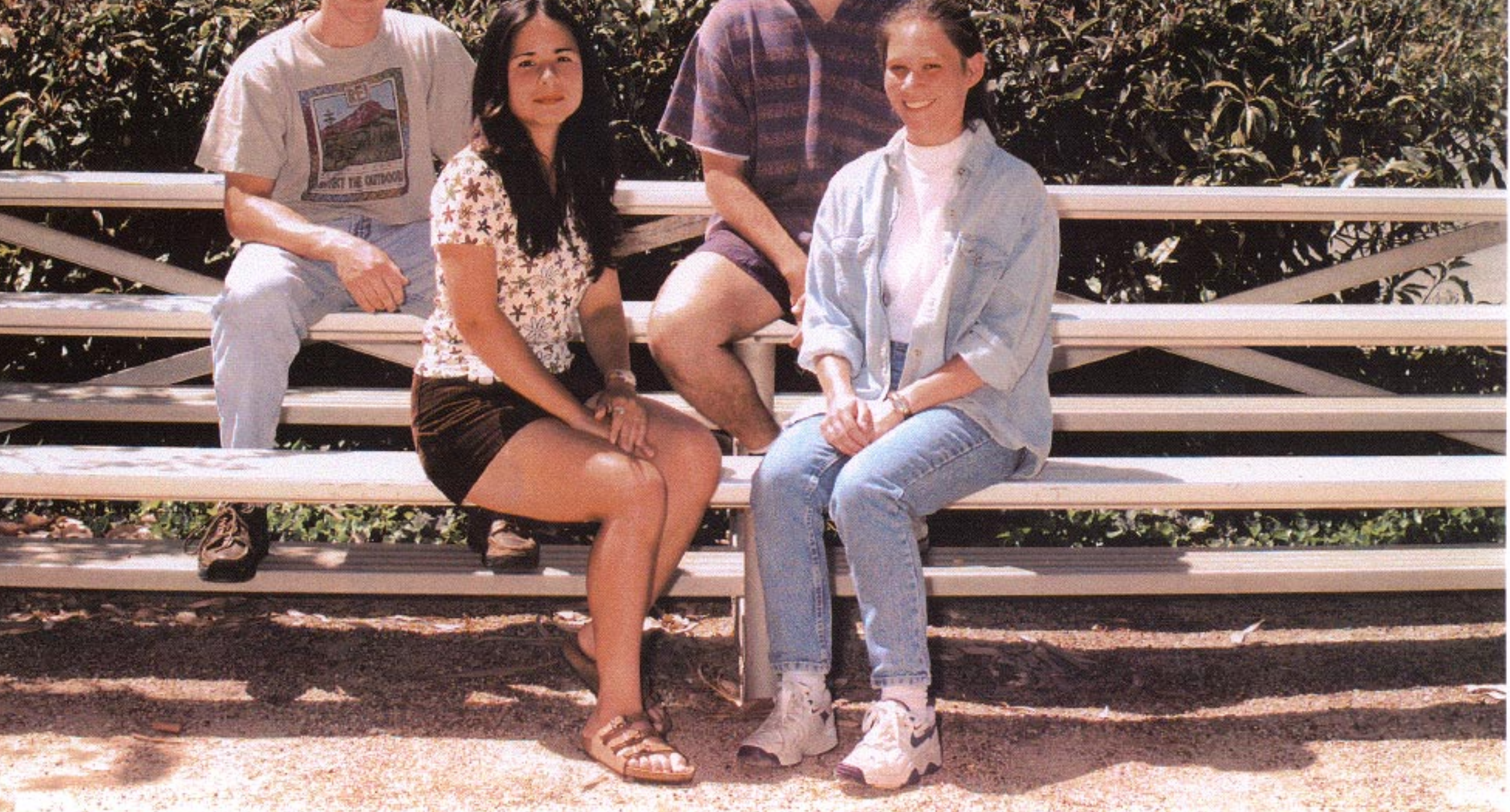




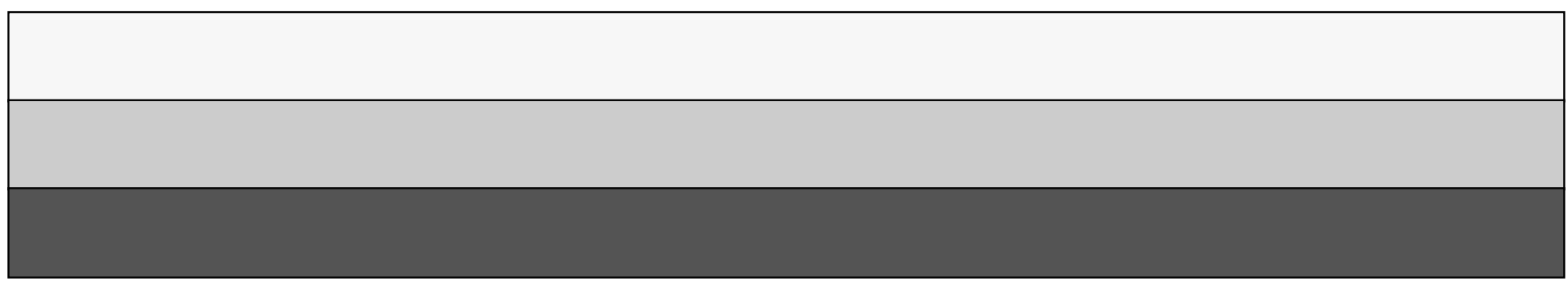

